2009

\title{
Gas-Kinetic Schemes for Direct Numerical Simulations of Compressible Homogeneous Turbulence
}

\author{
Wei Liao \\ Old Dominion University \\ Yan Peng \\ Old Dominion University, ypeng@odu.edu \\ Li-Shi Luo \\ Old Dominion University, lluo@odu.edu
}

Follow this and additional works at: https://digitalcommons.odu.edu/mathstat_fac_pubs

Part of the Fluid Dynamics Commons, Mathematics Commons, and the Statistical, Nonlinear, and Soft Matter Physics Commons

\section{Repository Citation}

Liao, Wei; Peng, Yan; and Luo, Li-Shi, "Gas-Kinetic Schemes for Direct Numerical Simulations of Compressible Homogeneous Turbulence" (2009). Mathematics \& Statistics Faculty Publications. 10.

https://digitalcommons.odu.edu/mathstat_fac_pubs/10

\section{Original Publication Citation}

Liao, W., Peng, Y., \& Luo, L. S. (2009). Gas-kinetic schemes for direct numerical simulations of compressible homogeneous turbulence. Physical Review E, 80(4), 046702. doi:10.1103/PhysRevE.80.046702 


\title{
Gas-kinetic schemes for direct numerical simulations of compressible homogeneous turbulence
}

\author{
Wei Liao, ${ }^{*}$ Yan Peng, ${ }^{\dagger}$ and Li-Shi Luo ${ }^{\ddagger}$ \\ Department of Mathematics and Statistics and Center for Computational Sciences, Old Dominion University, \\ Norfolk, Virginia 23529, USA
}

(Received 12 June 2009; published 14 October 2009)

\begin{abstract}
We apply the gas-kinetic scheme (GKS) for the direct numerical simulations (DNSs) of compressible decaying homogeneous isotropic turbulence (DHIT). We intend to study the accuracy, stability, and efficiency of the gas-kinetic scheme for DNS of compressible homogeneous turbulence depending on both flow conditions and numerics. In particular, we study the GKS with multidimensional, quasi-one-dimensional, dimensional-splitting, and smooth-flow approximations. We simulate the compressible DHIT with the Taylor microscale Reynolds number $\operatorname{Re}_{\lambda}=72.0$ and the turbulence Mach number $\mathrm{Ma}_{\mathrm{t}}$ between 0.1 and 0.6. We compute the low-order statistical quantities including the total kinetic energy $K(t)$, the dissipation rate $\varepsilon(t)$, the skewness $S_{u}(t)$, and the flatness $F_{u}(t)$ of the velocity field $\boldsymbol{u}(\boldsymbol{x}, t)$. We assess the effects on the turbulence statistics due to the approximations made in the treatment of fluxes, the flux limiter, the accuracy of the interpolation, and the bulk viscosity. Our results show that the GKS is adequate for DNS of compressible homogeneous turbulence as far as the low-order turbulence statistics are concerned.
\end{abstract}

DOI: 10.1103/PhysRevE.80.046702

PACS number(s): 47.11.-j, 47.40.-x, 94.05.Lk, 47.45.Ab

\section{INTRODUCTION}

Compressible turbulence has been a subject of interest for a long time [1-14]. In compressible turbulence, the Kolmogorov paradigm, which forms the basis of most equilibrium turbulence models, is questionable. This is due to the fact that there can be baroclinic-type production of energy at all scales of turbulence, including the inertial range, invalidating the Kolmogorov hypotheses. Consequently, the premises of most existing closure models for turbulence may not be valid any more, thus the turbulent mass, energy and momentum transport in compressible turbulence may not be amenable to standard treatment. Hence, physics-based modeling of compressible turbulence must start from fundamental first principles.

Recently, there has been an intense effort to use direct numerical simulation (DNS) for shock/turbulent boundary layer interactions (STBLI) (cf., e.g., [15-19] and a recent survey [20]), which are critically important for high-speed flows. The hope is that by vigorously interrogating data generated by high-fidelity DNS, one can gain insights into flow physics, which can in turn provide guidelines for turbulence modeling. To ensure high fidelity of DNS for compressible turbulent flows, the numerical methods are required to have minimal numerical dissipation and high bandwidth efficiency. These requirements naturally favor high-order methods. However, when shocks are considered, these requirements are more difficult to satisfy because they conflict with the stability requirement for shock capturing. The general solution to this conflict is a judicious addition of numerical dissipation in the neighborhood of shocks, which are treated as discontinuities (Godunov type of approaches [21-23]), while maintaining the desired (high) order of accuracy in smooth flow regions.

\footnotetext{
*wliao@odu.edu

†ypeng@odu.edu

†1luo@odu.edu
}

For compressible homogeneous isotropic turbulence in three dimensions, high-order methods which have been used for the purpose of DNS include the pseudospectral (PS) method $[2,3,6,7]$, the high-order compact finite-difference scheme $[4,10,13,24]$, and the fourth-order weighted essential nonoscillatory (WENO) scheme [25-27]. For homogeneous turbulence with high turbulence Mach number, shocklets are ubiquitous, and the Godunov-type methods become nominally first-order accurate across discontinuities, regardless of the order of accuracy of the interpolation used in reconstruction. Whether high-order schemes are more accurate and efficient than second-order schemes for flows with discontinuities is a subject of ongoing debate $[20,28,29]$.

In this study we will use the gas-kinetic scheme (GKS) $[30,31]$ for direct numerical simulations of the compressible decaying homogeneous isotropic turbulence (DHIT). The GKS is a second-order finite-volume kinetic scheme derived from the Boltzmann equation as opposed to conventional methods of computational fluid dynamics (CFD) based on discretizations of the Navier-Stokes equations. In contrast to conventional CFD methods, kinetic methods have two distinctive features. First, kinetic methods have the potential to include extended hydrodynamics beyond the validity of the Navier-Stokes equations because kinetic methods are based on kinetic theory and the Boltzmann equation, which provide the theoretical connection between hydrodynamics and the underlying microscopic physics. And, second, the Boltzmann equation is a first-order integro-partial-differential equation with a linear advection term, while the Navier-Stokes equation is a second-order partial differential equation with a nonlinear advection term. The nonlinearity in the Boltzmann equation resides in its collision term, which is local. This feature may lead to some computational advantages [32].

Compared to most conventional CFD methods, the GKS is relatively new and is still a topic of active research (e.g., $[31,33-40])$. Despite the fact that the GKS has been applied to simulate a wide variety of flow problems, such as nonequilibrium hypersonic flows with STBLIs [35,39,41], shock structures in gases [40,42-45], scalar transport and mixing in compressible flows [46-49], chemically reacting multicom- 
ponent compressible flows [50-52], nonequilibrium microflows $[53,54]$, magnetohydrodynamics $[55,56]$, and solutions of the shallow water equation [57-60], little has been done in the application of the GKS for DNS of compressible homogeneous turbulence [61]. It is well known that DNS of homogeneous turbulence are particularly sensitive to, among other things, numerical dissipations and thus are challenging for a second-order method.

The main objective of this work is to investigate the numerical accuracy, stability, and efficiency of the GKS for DNS of compressible DHIT in three dimensions. We will investigate the effects due to the approximations in the flux calculations, the flux limiters, the accuracy of the interpolation in reconstruction, and the bulk viscosity on the loworder statistical turbulence quantities, which include the kinetic energy $K(t)$, the dissipation rate $\varepsilon(t)$, and the skewness $S_{u}(t)$ and the flatness $F_{u}(t)$ of the velocity field $\boldsymbol{u}(\boldsymbol{x}, t)$. We will simulate compressible DHIT with the initial turbulence Mach number $\mathrm{Ma}_{\mathrm{t}}$ ranging from 0.1 to 0.6 , corresponding to near incompressible to fully compressible flow regions.

The remaining part of this paper is organized as follows. In Sec. II we discuss in detail (a) the construction of the full multidimensional (MD) GKS; (b) the quasi-one-dimensional (Q1D) and the dimensional-splitting (DS) gas-kinetic schemes, which are simpler thus more efficient than the full GKS; (c) the simplified GKS for smooth (incompressible) flows which is considerably simpler and efficient than the full GKS; and (d) the limiter, the interpolations used in the reconstruction, and the bulk viscosity in the GKS. In Sec. III we discuss the governing equations and flow conditions for the compressible DHIT, as well as the low-order statistical turbulence quantities to be computed. We also show some testing results to validate our code. In Sec. IV we present our main results. We first evaluate the necessity of using the full multidimensional GKS by comparing the results obtained with the Q1D-GKS, DS-GKS, and full MD-GKS. We test the Mach-number limit in the simplified GKS for smooth flows. We next investigate the effects due to the flux limiter, the interpolation, and the bulk viscosity on the low-order turbulence statistics. Finally, we conclude the paper in Sec. V.

\section{GAS-KINETIC SCHEME}

In this section we provide the details in the construction of the full MD GKS and several simplified versions of it, including the Q1D, DS, and the simplified GKS for smooth flows. We also discuss artificial dissipation, flux limiter, interpolations at cell boundaries, and the bulk viscosity in the GKS. We intend to provide sufficient details here so the GKS can be easily implemented and the results can be easily reproduced by our readers.

\section{A. Construction of gas-kinetic scheme}

To construct the full multidimensional gas-kinetic scheme for compressible flows $[30,31,39,40]$, we begin with the linearized Boltzmann equation (cf., e.g., [62]):

$$
\partial_{t} f+\boldsymbol{\xi} \cdot \nabla f=\mathcal{L}(f, f),
$$

where $f:=f(\boldsymbol{x}, \boldsymbol{\xi}, \boldsymbol{\zeta}, t)$ is the single particle distribution function of space $\boldsymbol{x}$, the particle velocity $\boldsymbol{\xi}:=\dot{\boldsymbol{x}}$, the particle inter- nal degrees of freedom $\zeta$ of $Z$ dimensions, and the time $t ; \mathcal{L}$ is the linearized collision operator. For the sake of simplicity and without losing generality in the context of the linearized Boltzmann equation, we will use the Bhatnagar-Gross-Krook (BGK) single relaxation-time model for $\mathcal{L}$ [63]:

$$
\partial_{t} f+\boldsymbol{\xi} \cdot \nabla f=-\frac{1}{\tau}\left[f-f^{(0)}\right],
$$

where $\tau$ is the relaxation time related to the mean free time between successive collisions and $f^{(0)}$ is the Maxwellian equilibrium distribution function of $D$ dimensions,

$$
f^{(0)}=\rho\left(\frac{\beta}{2 \pi}\right)^{(D+Z) / 2} \exp \left[-\frac{1}{2} \beta(\boldsymbol{c} \cdot \boldsymbol{c}+\boldsymbol{\zeta} \cdot \boldsymbol{\zeta})\right],
$$

where $\boldsymbol{c}:=(\boldsymbol{\xi}-\boldsymbol{u})$ is the peculiar velocity, $\beta=(R T)^{-1}, R$ is the gas constant, and $\rho, \boldsymbol{u}$ and $T$ are the density, flow velocity, and temperature, respectively.

By integrating along the characteristics [64], one can obtain the following solution of the BGK equation (2):

$$
f(\boldsymbol{x}+\boldsymbol{\xi} t, t)=e^{-t / \tau} f_{0}+\frac{1}{\tau} \int_{0}^{t} f^{(0)}\left(\boldsymbol{x}^{\prime}, \boldsymbol{\xi}, \boldsymbol{\zeta}, t^{\prime}\right) e^{\left(t^{\prime}-t\right) / \tau} d t^{\prime},
$$

where $\boldsymbol{x}^{\prime}:=\boldsymbol{x}+\boldsymbol{\xi} t^{\prime}$, and the initial state $f_{0}:=f(\boldsymbol{x}, \boldsymbol{\xi}, \boldsymbol{\zeta}, t=0)$. The GKS is formulated based on the above equation. With $f_{0}$ and $f_{0}^{(0)}:=f^{(0)}(\boldsymbol{x}, \boldsymbol{\xi}, \boldsymbol{\zeta}, t=0)$ given, one can construct an approximate solution for $f$ at any time $t \neq 0$. The gas-kinetic scheme is a finite-volume method for compressible flows. Thus, the values of the conserved variables are given at cell centers, while the values of fluxes are needed at cell boundaries. Unlike conventional CFD methods which evaluate fluxes from the hydrodynamic variables, the gas-kinetic scheme computes the numerical fluxes from the distribution function $f$.

For the sake of convenience, we shall use the following notation for the vectors of $(D+Z)$ dimensions:

$$
\begin{gathered}
\boldsymbol{\Psi}:=\left(1, \boldsymbol{\xi},\left(\xi^{2}+\zeta^{2}\right) / 2\right)^{\dagger}, \\
\mathbf{W}:=(\rho, \rho \boldsymbol{u}, \rho E)^{\dagger}=\int f \boldsymbol{\Psi} d \Xi=\int f^{(0)} \boldsymbol{\Psi} d \Xi, \\
\mathbf{F}_{\alpha}:=\int f \boldsymbol{\Psi} \xi_{\alpha} d \Xi, \quad \alpha \in\{x, y, z\}:=\{1,2,3\}, \\
\mathbf{h}:=(\rho, \boldsymbol{u}, T)^{\dagger}, \\
\mathbf{h}^{\prime}:=\left(\rho^{-1}, \beta \boldsymbol{u}, \frac{1}{2}\left[\beta\left(c^{2}+\zeta^{2}\right)-(D+Z)\right] T^{-1}\right)^{\dagger},
\end{gathered}
$$

where $\dagger$ denotes the transpose operation; $\boldsymbol{\Psi}, \mathbf{W}, \mathbf{F}_{\alpha}$, and $\mathbf{h}$ have the collisional invariants, the conserved quantities, the fluxes along the $\alpha$ axis, and the primitive variables as their components, respectively; $E$ is the specific total energy, $\rho E$ $=\rho \epsilon+\frac{1}{2} \rho \boldsymbol{u}^{2}$, where $\epsilon:=\frac{1}{2}(D+Z) R T$ is the specific internal energy and $R$ is the gas constant; and $\Xi:=(\boldsymbol{\xi}, \zeta)$ denotes the single particle velocity space and the internal degrees of freedom. According to Eq. (5b), the conserved variables are the 
conserved moments of the collision operator. In this work we will study three-dimensional (3D) flows in which the total number of internal degrees of freedom is $Z=(5-3 \gamma) /(\gamma$ $-1)$ and $\gamma=c_{p} / c_{v}$ is the ratio of specific heats.

For a finite-volume scheme to be truly multidimensional, the gradients of flow variables in both normal and two tangential directions at a cell interface must be considered impartially. This can be easily achieved in the GKS method because the advection term $\boldsymbol{\xi} \cdot \boldsymbol{\nabla} f:=\boldsymbol{\nabla} \cdot(\boldsymbol{\xi} f)$ in the Boltzmann equation is linear, thus operator splitting among $D$ coordinates can be easily implemented. To simplify the ensuing discussion, we will show the construction of fluxes in the GKS method along one direction, say $x$, for construction of fluxes along the other two directions can be done similarly. We denote a cell center by $\boldsymbol{x}_{i, j, k}$, and its left and right cell boundaries along $x$ coordinate by $\boldsymbol{x}_{i-1 / 2, j, k}$ and $\boldsymbol{x}_{i+1 / 2, j, k}$, respectively. For simplicity, we set the initial time $t_{0}=0$, then solution (4) at position $\boldsymbol{x}_{i+1 / 2, j, k}$ and time $t$ is

$$
\begin{aligned}
f\left(\boldsymbol{x}_{i+1 / 2, j, k}, t\right)= & e^{-t / \tau} f_{0}\left(\boldsymbol{x}_{i+1 / 2, j, k}-\boldsymbol{\xi} t\right) \\
& +\frac{1}{\tau} \int_{0}^{t} f^{(0)}\left(\boldsymbol{x}^{\prime}, t^{\prime}\right) e^{-\left(t-t^{\prime}\right) / \tau} d t^{\prime},
\end{aligned}
$$

where $\boldsymbol{x}^{\prime}:=\boldsymbol{x}_{i+1 / 2, j, k}-\boldsymbol{\xi}\left(t-t^{\prime}\right)$ is the coordinate of the particle trajectory. In the above equation, we omit the variables $\boldsymbol{\xi}$ and $\zeta$ in $f$ whenever they remain constant. Initially, only the values of the conserved variables $\rho, \rho \boldsymbol{u}$, and $\rho E$ are given at the cell center $\boldsymbol{x}_{i, j, k}$, but the fluxes are to be evaluated at the cell boundaries $\boldsymbol{x}_{i \pm 1 / 2, j, k}=0$. Therefore, both $f_{0}$ and $f^{(0)}\left(x^{\prime}, t^{\prime}\right)$ in the above equation are to be constructed from the hydrodynamic variables through the Boltzmann equation and Taylor expansions of $f$.

We can formally write the BGK equation (2) as the following:

$$
f=f^{(0)}-\tau d_{t} f, \quad d_{t}:=\partial_{t}+\boldsymbol{\xi} \cdot \boldsymbol{\nabla} .
$$

Thus, $f$ can be solved iteratively, starting with $f=f^{(0)}$ on the right-hand side of the above equation. For the purpose of simulating the Navier-Stokes equation, $f=f^{(0)}-\tau d_{t} f^{(0)}$ is sufficient. The initial value can be approximated as

$$
\begin{aligned}
f_{0}(\boldsymbol{x}, 0) \approx & {\left[1-\tau\left(\partial_{t}+\boldsymbol{\xi} \cdot \boldsymbol{\nabla}\right)\right] f^{(0)}(\boldsymbol{x}, 0)=\left[1-\boldsymbol{h}^{\prime} \cdot\left(\partial_{t}\right.\right.} \\
& +\boldsymbol{\xi} \cdot \boldsymbol{\nabla}) \mathbf{h}] f^{(0)}(\boldsymbol{x}, 0) .
\end{aligned}
$$

In addition, the equilibrium can be expanded in a Taylor series about $\boldsymbol{x}=\mathbf{0}$,

$$
f^{(0)}(\boldsymbol{x}, 0) \approx(1+\boldsymbol{x} \cdot \boldsymbol{\nabla}) f^{(0)}(0,0)=\left[1+\mathbf{h}^{\prime} \cdot(\boldsymbol{x} \cdot \boldsymbol{\nabla}) \mathbf{h}\right] f^{(0)}(0,0),
$$

where $\boldsymbol{x}:=(x, y, z)$. By substituting Eq. (9) into Eq. (8), we have

$$
\begin{aligned}
f_{0}(\boldsymbol{x}, 0) & \approx\left[1+\mathbf{h}^{\prime} \cdot(\boldsymbol{x} \cdot \boldsymbol{\nabla}) \mathbf{h}\right]\left[1-\boldsymbol{h}^{\prime} \cdot\left(\partial_{t}+\boldsymbol{\xi} \cdot \boldsymbol{\nabla}\right) \mathbf{h}\right] f^{(0)}(0,0) \\
& =[1+\boldsymbol{a} \cdot(\boldsymbol{x}-\boldsymbol{\xi} \tau)-A \tau] f^{(0)}(0,0),
\end{aligned}
$$

where $\quad \boldsymbol{a}:=\left(a_{1}, a_{2}, a_{3}\right):=\left(\mathbf{h}^{\prime} \cdot \partial_{x} \mathbf{h}, \mathbf{h}^{\prime} \cdot \partial_{y} \mathbf{h}, \mathbf{h}^{\prime} \cdot \partial_{z} \mathbf{h}\right)$ $:=\mathbf{h}^{\prime} \cdot\left(\partial_{x} \mathbf{h}, \partial_{y} \mathbf{h}, \partial_{z} \mathbf{h}\right)$ and $A=\mathbf{h}^{\prime} \cdot \partial_{t} \mathbf{h}$ are functions of $\boldsymbol{\xi}$ and $\boldsymbol{\zeta}$, and the hydrodynamic variables $\rho, \boldsymbol{u}$, and $T$, and their first- order derivatives. The coefficients $\boldsymbol{a}$ and $A$ are related by the compatibility condition for $f$

$$
\int f^{(n)} \boldsymbol{\Psi} d \boldsymbol{\Xi}=\mathbf{0}, \quad \forall n>0,
$$

where $f^{(n)}$ is the $n$ th-order Chapman-Enskog expansion of $f$ and $f^{(0)}$ is the Maxwellian given by Eq. (9). Therefore, the first-order compatibility condition

$$
\begin{aligned}
\int f^{(1)} \boldsymbol{\Psi} d \Xi & =-\tau \int d_{t} f^{(0)} \boldsymbol{\Psi} d \Xi=-\tau \int(A+\boldsymbol{a} \cdot \boldsymbol{\xi}) f^{(0)} \boldsymbol{\Psi} d \Xi \\
& =0
\end{aligned}
$$

leads to the relation between $A$ and $\boldsymbol{a}:=\left(a_{1}, a_{2}, a_{3}\right)$,

$$
\int A f^{(0)} \boldsymbol{\Psi} d \boldsymbol{\Xi}=-\int \boldsymbol{a} \cdot \boldsymbol{\xi} f^{(0)} \mathbf{\Psi} d \boldsymbol{\Xi} .
$$

We can concisely write the end results of $\boldsymbol{a}=\boldsymbol{\nabla} \ln f^{(0)}$ and $A=\partial_{t} \ln f^{(0)}$ as following [65]:

$$
\begin{gathered}
\boldsymbol{a}=\boldsymbol{\nabla} \ln \rho+\left[\frac{\left(c^{2}+\zeta^{2}\right)}{2 R T}-\frac{3+Z}{2}\right] \boldsymbol{\nabla} \ln T+\frac{1}{R T} \sum_{\alpha=1}^{3} c_{\alpha} \nabla u_{\alpha} \\
A=-\boldsymbol{a} \cdot \boldsymbol{\xi}+\left[\frac{\left(c^{2}+\zeta^{2}\right)}{2 R T}-\frac{5+Z}{2}\right] \boldsymbol{c} \cdot \boldsymbol{\nabla} \ln T \\
+\frac{1}{R T}\left[\boldsymbol{c} \boldsymbol{c}-\frac{1}{5}\left(c^{2}+\zeta^{2}\right) \mathbf{I}\right]: \boldsymbol{\nabla} \boldsymbol{~}
\end{gathered}
$$

where $\boldsymbol{c}:=(\boldsymbol{\xi}-\boldsymbol{u})$ is the peculiar velocity, $c^{2}:=\boldsymbol{c} \cdot \boldsymbol{c}$, and $\mathbf{I}$ is the $3 \times 3$ identity matrix. For fully compressible flows, the conserved variables $(\rho, \rho \boldsymbol{u}, \rho E)$ are used as opposed to the primitive ones $(\rho, \boldsymbol{u}, T)$. The Jacobians between the primitive and conserved variables are readily available to transfer one set of variables to the other. It should also be noted that in computing the gradients $\partial_{\alpha} \mathbf{h}$ for the coefficients $\boldsymbol{a}$ $:=\left(a_{1}, a_{2}, a_{3}\right)$ in Eq. (10), we should allow the hydrodynamic variables to be discontinuous at the cell boundary $\boldsymbol{x}_{i \pm 1 / 2, j, k}$ in general for compressible flows.

As for $f^{(0)}(\boldsymbol{x}, t)$ in the integrand of Eq. (6), it can be evaluated by its Taylor expansion,

$$
\begin{aligned}
f^{(0)}(\boldsymbol{x}, t) \approx & \left(1+t \partial_{t}+\boldsymbol{x} \cdot \boldsymbol{\nabla}\right) f^{(0)}(0,0)=f^{(0)}(0,0)\left[1+\mathbf{h}^{\prime} \cdot\left(t \partial_{t}\right.\right. \\
& +\boldsymbol{x} \cdot \boldsymbol{\nabla}) \mathbf{h}]=(1+\overline{\boldsymbol{a}} \cdot \boldsymbol{x}+\bar{A} t) f^{(0)}(0,0),
\end{aligned}
$$

where $\overline{\boldsymbol{a}}:=\left(\bar{a}_{1}, \bar{a}_{2}, \bar{a}_{3}\right)$ and $\bar{A}$ are similar to $\boldsymbol{a}:=\left(a_{1}, a_{2}, a_{3}\right)$ and $A$, respectively. The difference is that in $\overline{\boldsymbol{a}}:=\left(\bar{a}_{1}, \bar{a}_{2}, \bar{a}_{3}\right)$, the hydrodynamic variables themselves are assumed to be continuous, but not their gradients in the directions normal to cell interfaces, while in $\boldsymbol{a}:=\left(a_{1}, a_{2}, a_{3}\right)$, both the hydrodynamic variables and their gradients are allowed to be discontinuous. That means that, for example, in $\bar{a}_{1}$ the gradients of hydrodynamic variables are allowed to be discontinuous when computing $\partial_{x} \mathbf{h}$. The details about how to evaluate $\boldsymbol{a}$ $:=\left(a_{1}, a_{2}, a_{3}\right), A, \overline{\boldsymbol{a}}:=\left(\bar{a}_{1}, \bar{a}_{2}, \bar{a}_{3}\right)$, and $\bar{A}$ will be further discussed next. 
Assuming the hydrodynamic variables are discontinuous at the cell boundary of $\boldsymbol{x}_{i+1 / 2, j, k}=0$, then the values of the equilibrium $f^{(0)}$ on both sides of the cell boundary have to be evaluated differently. For the value $f_{\mathrm{L}}^{(0)}$ on the left side, the hydrodynamic variables $\mathbf{h}$ are interpolated to the left cell boundary $\boldsymbol{x}_{i+1 / 2, j, k}^{-}$with two points left of and one point right of $\boldsymbol{x}_{i+1 / 2, j, k}$, i.e., $\boldsymbol{x}_{i-1, j, k}, \boldsymbol{x}_{i, j, k}$ and $\boldsymbol{x}_{i+1, j, k}$. Then the left equilibrium value $f_{\mathrm{L}}^{(0)}$ is computed from the hydrodynamic variables at $\boldsymbol{x}_{i+1 / 2, j, k}^{-}$. Similarly, the right equilibrium value $f_{\mathrm{R}}^{(0)}$ is evaluated from the hydrodynamic variables interpolated to $\boldsymbol{x}_{i+1 / 2, j, k}^{+}$with two points right of and one point left of $\boldsymbol{x}_{i+1 / 2, j, k}$, i.e., $\boldsymbol{x}_{i, j, k}, \boldsymbol{x}_{i+1, j, k}$, and $\boldsymbol{x}_{i+2, j, k}$. The van Leer limiter is used in the interpolations to suppress the spurious oscillations $[31,66]$ :

$$
\begin{gathered}
\frac{\Delta W_{i}}{\Delta x}=\left[\operatorname{sign}\left(W^{+}\right)+\operatorname{sign}\left(W^{-}\right)\right] \frac{\left|W^{+}\right|\left|W^{-}\right|}{\left|W^{+}\right|+\left|W^{-}\right|}, \\
W_{i+1 / 2}^{\mathrm{R}}=W_{i+1}-\frac{\Delta W_{i+1}}{\Delta x}\left(x_{i+1}-x_{i+1 / 2}\right), \\
W_{i+1 / 2}^{\mathrm{L}}=W_{i}+\frac{\Delta W_{i}}{\Delta x}\left(x_{i+1 / 2}-x_{i}\right),
\end{gathered}
$$

where $\Delta W_{i} / \Delta x$ denotes the approximated gradient in $x$ direction of the conserved variable $W$ at the $i$ th cell center, $W^{+}:=\left(W_{i+1}-W_{i}\right) / \Delta x$ and $W^{-}:=\left(W_{i}-W_{i-1}\right) / \Delta x$.

Specifically, the gradients of the hydrodynamic variables at the left cell boundary are computed as the following:

$$
\begin{gathered}
\partial_{x} \mathbf{h}_{\mathrm{L}}\left(\boldsymbol{x}_{i+1 / 2, j, k}^{-}\right)=\frac{\mathbf{h}\left(\boldsymbol{x}_{i+1 / 2, j, k}^{-}\right)-\mathbf{h}\left(\boldsymbol{x}_{i, j, k}\right)}{x_{i+1 / 2, j, k}-x_{i, j, k}}, \\
\partial_{y} \mathbf{h}_{\mathrm{L}}\left(\boldsymbol{x}_{i+1 / 2, j, k}^{-}\right)=\frac{\mathbf{h}\left(\boldsymbol{x}_{i+1 / 2, j+1, k}^{-}\right)-\mathbf{h}\left(\boldsymbol{x}_{i+1 / 2, j-1, k}^{-}\right)}{y_{i+1 / 2, j+1, k}-y_{i+1 / 2, j-1, k}}, \\
\partial_{z} \mathbf{h}_{\mathrm{L}}\left(\boldsymbol{x}_{i+1 / 2, j, k}^{-}\right)=\frac{\mathbf{h}\left(\boldsymbol{x}_{i+1 / 2, j, k+1}^{-}\right)-\mathbf{h}\left(\boldsymbol{x}_{i+1 / 2, j, k-1}^{-}\right)}{z_{i+1 / 2, j, k+1}-z_{i+1 / 2, j, k-1}} .
\end{gathered}
$$

Then the coefficients $\boldsymbol{a}_{\mathrm{L}}:=\left(a_{1 \mathrm{~L}}, a_{2 \mathrm{~L}}, a_{3 \mathrm{~L}}\right)$ at the left cell boundary $\boldsymbol{x}_{i+1 / 2, j, k}^{-}$are given by

$$
\begin{aligned}
& a_{1 \mathrm{~L}}\left(\boldsymbol{x}_{i+1 / 2, j, k}^{-}\right)=\mathbf{h}_{\mathrm{L}}^{\prime}\left(\boldsymbol{x}_{i+1 / 2, j, k}^{-}\right) \cdot \partial_{x} \mathbf{h}_{\mathrm{L}}\left(\boldsymbol{x}_{i+1 / 2, j, k}^{-}\right), \\
& a_{2 \mathrm{~L}}\left(\boldsymbol{x}_{i+1 / 2, j, k}^{-}\right)=\mathbf{h}_{\mathrm{L}}^{\prime}\left(\boldsymbol{x}_{i+1 / 2, j, k}^{-}\right) \cdot \partial_{y} \mathbf{h}_{\mathrm{L}}\left(\boldsymbol{x}_{i+1 / 2, j, k}^{-}\right), \\
& a_{3 \mathrm{~L}}\left(\boldsymbol{x}_{i+1 / 2, j, k}^{-}\right)=\mathbf{h}_{\mathrm{L}}^{\prime}\left(\boldsymbol{x}_{i+1 / 2, j, k}^{-}\right) \cdot \partial_{z} \mathbf{h}_{\mathrm{L}}\left(\boldsymbol{x}_{i+1 / 2, j, k}^{-}\right) .
\end{aligned}
$$

Similarly, at the right cell boundary $\boldsymbol{x}_{i+1 / 2, j, k}^{+}$, the hydrodynamic variables are interpolated from the following three points: $\boldsymbol{x}_{i, j, k}, \boldsymbol{x}_{i+1, j, k}$, and $\boldsymbol{x}_{i+2, j, k}$, and we have

$$
\begin{gathered}
\partial_{x} \mathbf{h}_{\mathrm{L}}\left(\boldsymbol{x}_{i+1 / 2, j, k}^{+}\right)=\frac{\mathbf{h}\left(\boldsymbol{x}_{i+1 / 2, j, k}^{+}\right)-\mathbf{h}\left(\boldsymbol{x}_{i+1, j, k}\right)}{x_{i+1 / 2, j, k}-x_{i+1, j, k}}, \\
\partial_{y} \mathbf{h}_{\mathrm{L}}\left(\boldsymbol{x}_{i+1 / 2, j, k}^{+}\right)=\frac{\mathbf{h}\left(\boldsymbol{x}_{i+1 / 2, j+1, k}^{+}\right)-\mathbf{h}\left(\boldsymbol{x}_{i+1 / 2, j-1, k}^{+}\right)}{y_{i+1 / 2, j+1, k}-y_{i+1 / 2, j-1, k}},
\end{gathered}
$$

$$
\partial_{z} \mathbf{h}_{\mathrm{L}}\left(\boldsymbol{x}_{i+1 / 2, j, k}^{+}\right)=\frac{\mathbf{h}\left(\boldsymbol{x}_{i+1 / 2, j, k+1}^{+}\right)-\mathbf{h}\left(\boldsymbol{x}_{i+1 / 2, j, k-1}^{+}\right)}{z_{i+1 / 2, j, k+1}-z_{i+1 / 2, j, k-1}},
$$

and coefficients $\boldsymbol{a}_{\mathrm{R}}:=\left(a_{1 \mathrm{R}}, a_{2 \mathrm{R}}, a_{3 \mathrm{R}}\right)$ can be calculated similarly to $\boldsymbol{a}_{\mathrm{L}}:=\left(a_{1 \mathrm{~L}}, a_{2 \mathrm{~L}}, a_{3 \mathrm{~L}}\right)$, except that they are computed at $\boldsymbol{x}_{i+1 / 2, j+1, k}^{+}$, instead of $\boldsymbol{x}_{i+1 / 2, j+1, k}^{-}$, in Eq. (17). With $\boldsymbol{a}_{\mathrm{L}}$ $:=\left(a_{1 \mathrm{~L}}, a_{2 \mathrm{~L}}, a_{3 \mathrm{~L}}\right)$ and $\boldsymbol{a}_{\mathrm{R}}:=\left(a_{1 \mathrm{R}}, a_{2 \mathrm{R}}, a_{3 \mathrm{R}}\right)$ given, $A_{\mathrm{L}}$ and $A_{\mathrm{R}}$ can be obtained immediately by using the compatibility condition (12).

The equilibria at the both sides of the cell boundary $\boldsymbol{x}_{i+1 / 2, j, k}$ are $f_{\mathrm{L}}^{(0)}:=f^{(0)}\left(\boldsymbol{\xi}, \mathbf{h}_{\mathrm{L}}\right)$ and $f_{\mathrm{R}}^{(0)}:=f^{(0)}\left(\boldsymbol{\xi}, \mathbf{h}_{\mathrm{R}}\right)$, which are available now because $\mathbf{h}_{\mathrm{L}}$ and $\mathbf{h}_{\mathrm{R}}$ are given. At the equilibrium $f^{(0)}$, the hydrodynamic variables are assumed to be continuous. Therefore, the conservative variables $\mathbf{W}$ at the cell boundary $\boldsymbol{x}_{i+1 / 2, j, k}$ are obtained by integrating the equilibrium at both sides of the cell boundary $\boldsymbol{x}_{i+1 / 2, j, k}$ :

$$
\mathbf{W}\left(\boldsymbol{x}_{i+1 / 2, j, k}\right)=\int_{\xi_{x} \geq 0} d \Xi \boldsymbol{\Psi} f_{\mathrm{L}}^{(0)}+\int_{\xi_{x} \leq 0} d \Xi \boldsymbol{\Psi} f_{\mathrm{R}}^{(0)},
$$

the hydrodynamic variables $\mathbf{h}:=(\rho, \boldsymbol{u}, T)^{\dagger}$ can be easily obtained from the conservative variables $\mathbf{W}:=(\rho, \rho \boldsymbol{u}, \rho E)^{\dagger}$, and then the coefficients $\bar{a}_{1 \mathrm{~L}}$ and $\bar{a}_{1 \mathrm{R}}$ are computed as the following:

$$
\begin{gathered}
\bar{a}_{1 \mathrm{~L}}\left(\boldsymbol{x}_{i+1 / 2, j, k}\right)=\mathbf{h}^{\prime}\left(\boldsymbol{x}_{i+1 / 2, j, k}\right) \cdot \frac{\mathbf{h}\left(\boldsymbol{x}_{i+1 / 2, j, k}\right)-\mathbf{h}\left(\boldsymbol{x}_{i, j, k}\right)}{\boldsymbol{x}_{i+1 / 2, j, k}-\boldsymbol{x}_{i, j, k}}, \\
\bar{a}_{1 \mathrm{R}}\left(\boldsymbol{x}_{i+1 / 2, j, k}\right)=\mathbf{h}^{\prime}\left(\boldsymbol{x}_{i+1 / 2, j, k}\right) \cdot \frac{\mathbf{h}\left(x_{i+1 / 2, j, k}\right)-\mathbf{h}\left(x_{i+1, j, k}\right)}{\boldsymbol{x}_{i+1 / 2, j, k}-\boldsymbol{x}_{i+1, j, k}} .
\end{gathered}
$$

Consequently, we have

$$
\begin{aligned}
f_{0}(\boldsymbol{x}, 0)=f_{0 \mathrm{~L}}(\boldsymbol{x}, 0)+f_{0 \mathrm{R}}(\boldsymbol{x}, 0) & \\
= & H(-x)\left[1+\boldsymbol{a}_{\mathrm{L}} \cdot(\boldsymbol{x}-\boldsymbol{\xi} \tau)-\tau A_{\mathrm{L}}\right] f_{\mathrm{L}}^{(0)}(0,0) \\
+ & H(x)\left[1+\boldsymbol{a}_{\mathrm{R}} \cdot(\boldsymbol{x}-\boldsymbol{\xi} \tau)-\tau A_{\mathrm{R}}\right] f_{\mathrm{R}}^{(0)}(0,0), \\
f^{(0)}(\boldsymbol{x}, t)= & {\left[1+H(-x) \bar{a}_{1 \mathrm{~L}} x+H(x) \bar{a}_{1 \mathrm{R}} x+\bar{a}_{2} y\right.} \\
& \left.+\bar{a}_{3} z+\bar{A} t\right] f^{(0)}(0,0),
\end{aligned}
$$

where $H(x)$ is the Heaviside function. Finally, the value of $f$ at a cell boundary can be obtained by substituting the above equations of $f_{0}(\boldsymbol{x}, t)$ and $f^{(0)}(\boldsymbol{x}, t)$ into Eq. (6),

$$
\begin{aligned}
f\left(\boldsymbol{x}_{i+1 / 2, j, k}, t\right)=\left\{\left[(1-\bar{A} \tau)\left(1-e^{-t / \tau}\right)+\bar{A} t\right]\right. \\
\quad+\left[(t+\tau) e^{-t / \tau}-\tau\right]\left\{\left[\bar{a}_{1 \mathrm{~L}} H\left(\xi_{1}\right)\right.\right. \\
\left.\left.\left.\quad+\bar{a}_{1 \mathrm{R}} H\left(-\xi_{1}\right)\right] \xi_{1}+\bar{a}_{2} \xi_{2}+\bar{a}_{3} \xi_{3}\right\}\right\} f_{0}^{(0)} \\
+e^{-t / \tau}\left\{\left[1-(t+\tau) \boldsymbol{a}_{\mathrm{L}} \cdot \boldsymbol{\xi}-\tau A_{\mathrm{L}}\right] H\left(\xi_{1}\right) f_{0 \mathrm{~L}}^{(0)}\right. \\
\left.\quad+\left[1-(t+\tau) \boldsymbol{a}_{\mathrm{R}} \cdot \boldsymbol{\xi}-\tau A_{\mathrm{R}}\right] H\left(-\xi_{1}\right) f_{0 \mathrm{R}}^{(0)}\right\},
\end{aligned}
$$

where $f_{0}^{(0)}, f_{0 \mathrm{~L}}^{(0)}$, and $f_{0 \mathrm{R}}^{(0)}$ are initial values of $f^{(0)}, f_{\mathrm{L}}^{(0)}$, and $f_{\mathrm{R}}^{(0)}$ evaluated at the cell boundary $\boldsymbol{x}_{i+1 / 2, j, k}$. The only unknown in $f\left(\boldsymbol{x}_{i+1 / 2, j, k}, t\right)$ of Eq. (21) is the coefficient $\bar{A}$. By using $f^{(0)}\left(\boldsymbol{x}_{i+1 / 2, j, k}, t\right)$ of Eq. (20) and $f\left(\boldsymbol{x}_{i+1 / 2, j, k}, t\right)$ of Eq. (21), the conservation laws lead to the following equation: 


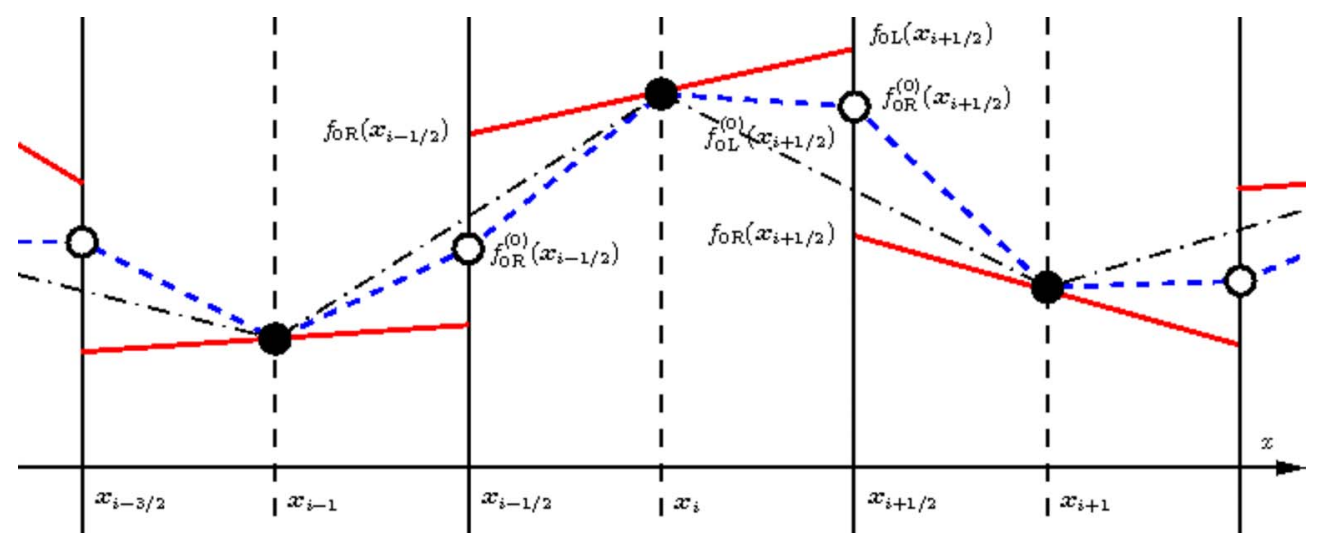

FIG. 1. (Color online) A 1D illustration of construction of $f$. Dashed and solid vertical lines indicate cell centers and boundaries, respectively. Discs and circles indicate the values of the equilibrium $f^{(0)}$ at cell centers and boundaries, which are given initially at $t=t_{0}$ and by Eq. (19), respectively. The continuous piecewise linear dash-dot line represents $f^{(0)}$, which is assumed to be linear between two cell-center values. The continuous piecewise linear dash line connecting the discs and circles and the discontinuous solid lines represent $f^{(0)}$ and $f_{0}$ obtained with a limiter, respectively.

$$
\int_{0}^{\Delta t} d t \int d \Xi \boldsymbol{\Psi} f^{(0)}\left(\boldsymbol{x}_{i+1 / 2, j, k}, t\right)=\int_{0}^{\Delta t} d t \int d \Xi \boldsymbol{\Psi} f\left(\boldsymbol{x}_{i+1 / 2, j, k}, t\right)
$$

which determines $\bar{A}$ in terms of spatial gradients of hydrodynamic variables: $\boldsymbol{a}_{\mathrm{L}}:=\left(a_{1 \mathrm{~L}}, a_{2 \mathrm{~L}}, a_{3 \mathrm{~L}}\right), \quad \boldsymbol{a}_{\mathrm{R}}:=\left(a_{1 \mathrm{R}}, a_{2 \mathrm{R}}, a_{3 \mathrm{R}}\right)$, $\bar{a}_{1 \mathrm{~L}}, \bar{a}_{1 \mathrm{R}}, \bar{a}_{2}$, and $\bar{a}_{3}$. Therefore, $f^{(0)}\left(\boldsymbol{x}_{i+1 / 2, j, k}, t\right)$ is determined from the hydrodynamic variables at the cell centers around the cell boundary $\boldsymbol{x}_{i+1 / 2, j, k}$. Figure 1 provides an 1D illustration of construction of the distribution function $f$ with a limiter.

A succinct comment concerning the multidimensionality of the GKS is in order at this point. Clearly, the flux in $x$ direction given by Eq. (21) includes the gradients of hydrodynamic variables in all directions, regardless of the mesh orientation. The only effect of mesh here is the accuracy with which the gradients are computed with a given mesh. This multidimensional feature saliently distinguishes the GKS from any Riemann solver based on the picture of onedimensional wave.

In the gas-kinetic scheme, the relaxation time $\tau$ in Eq. (21) is determined by the local hydrodynamic variables through

$$
\tau=\mu / p,
$$

where $\mu$ is the dynamic viscosity and $p$ is the pressure. The above relation between $\tau, \mu$, and $p$ is valid when hydrodynamic variables are continuous. When discontinuity is allowed as in compressible flows with shocks, artificial dissipation must be introduced to capture shocks [31]. The artificial dissipation is introduced in the GKS method by modifying the relaxation time $\tau$ as the following:

$$
\tau=\frac{\mu\left(x_{i+1 / 2}\right)}{p\left(x_{i+1 / 2}\right)}+\sigma \Delta t \frac{\left|p_{\mathrm{L}}-p_{\mathrm{R}}\right|}{\left(p_{\mathrm{L}}+p_{\mathrm{R}}\right)}=\tau_{\mu}+\sigma \tau_{\sigma},
$$

where $\tau_{\mu}$ and $\sigma \tau_{\sigma}$ represent the physical and artificial relaxation times, respectively. A detailed discussion about computing $\tau_{\mu}$ and $\sigma \tau_{\sigma}$ is referred to Sec. II D.
With $f$ given at the cell boundaries, the time-dependent fluxes can be evaluated,

$$
\begin{aligned}
\mathbf{F}_{x}^{i+1 / 2, j, k} & =\int \xi_{x} \Psi f\left(\boldsymbol{x}_{i+1 / 2, j, k}, t\right) d \Xi, \\
\mathbf{F}_{y}^{i, j+1 / 2, k} & =\int \xi_{y} \Psi f\left(\boldsymbol{x}_{i, j+1 / 2, k}, t\right) d \Xi, \\
\mathbf{F}_{z}^{i, j, k+1 / 2} & =\int \xi_{z} \Psi f\left(\boldsymbol{x}_{i, j, k+1 / 2}, t\right) d \Xi .
\end{aligned}
$$

Thus the fluxes $\mathbf{F}_{\alpha}$ are fully determined through the distribution function $f$ at the cell interfaces $\boldsymbol{x}_{i+1 / 2, j, k}, \boldsymbol{x}_{i, j+1 / 2, k}$, and $\boldsymbol{x}_{i, j, k+1 / 2}$. By integrating the above equation over each time step $\Delta t$, we obtain the total fluxes as

$$
\begin{aligned}
& \overline{\mathbf{F}}_{x}^{i \pm 1 / 2, j, k}=\int_{0}^{\Delta t} \mathbf{F}_{x}^{i \pm 1 / 2, j, k} d t, \\
& \overline{\mathbf{F}}_{y}^{i, j \pm 1 / 2, k}=\int_{0}^{\Delta t} \mathbf{F}_{y}^{i, j \pm 1 / 2, k} d t, \\
& \overline{\mathbf{F}}_{z}^{i, j, k \pm 1 / 2}=\int_{0}^{\Delta t} \mathbf{F}_{z}^{i, j, k \pm 1 / 2} d t .
\end{aligned}
$$

The GKS is an explicit numerical scheme and therefore its time step $\Delta t$ in Eq. (27) is dictated by local flow characteristics. For the viscous flows governed by Navier-Stokes equations, the time step is determined by the following Courant-Friedrichs-Lewy (CFL) condition:

$$
\Delta t \leq \frac{\Delta x \mu_{\mathrm{CFL}}}{\left(|\boldsymbol{u}|+c_{s}\right)\left(1+2 / \mathrm{Re}^{*}\right)},
$$

where $\mu_{\mathrm{CFL}}$ is the CFL number, $c_{s}=\sqrt{\gamma R T}$ is the speed of sound, and $\mathrm{Re}^{*}:=|\boldsymbol{u}| \Delta x / \nu$ is the grid Reynolds number. The governing equations in the finite-volume formulation can then be written as 


$$
\begin{aligned}
\mathbf{W}_{i j k}^{n+1}= & \mathbf{W}_{i j k}^{n}-\frac{1}{\Delta x}\left(\overline{\mathbf{F}}_{x}^{i+1 / 2, j, k}-\overline{\mathbf{F}}_{x}^{i-1 / 2, j, k}\right)-\frac{1}{\Delta y}\left(\overline{\mathbf{F}}_{y}^{i, j+1 / 2, k}\right. \\
& \left.-\overline{\mathbf{F}}_{y}^{i, j-1 / 2, k}\right)-\frac{1}{\Delta z}\left(\overline{\mathbf{F}}_{z}^{i, j, k+1 / 2}-\overline{\mathbf{F}}_{z}^{i, j, k-1 / 2}\right),
\end{aligned}
$$

which are used to update the conserved flow variables.

\section{B. Multidimensional, quasi-one-dimensional, and directional splitting GKS}

The particle velocity distribution function $f\left(\boldsymbol{x}_{i+1 / 2, j, k}, t\right)$ at a cell interface is computed according to Eq. (21), which in turn determines the fluxes at a cell interface. To make the gas-kinetic scheme multidimensional, the fluxes must consider, in principle, the gradients of flow variables in both normal and tangential directions at a cell interface. The flux calculation in the GKS method is more costly than most conventional CFD methods, thus one often invokes various approximations to enhance computational efficiency without thorough understanding and assessment of the effects of these approximations on physical fidelity of the GKS method. Specifically, we will discuss the approximations leading to the quasi-one-dimensional (Q1D) and the directional-splitting (DS) GKS schemes [31,39,48,57,67] and evaluate the effects of these approximations in comparison with the full multidimensional GKS scheme.

In both the Q1D-GKS and the DS-GKS, the fluxes computed at cell interfaces ignore gradients of flow variables tangential to cell interfaces, that is, in Eq. (21) of $f\left(\boldsymbol{x}_{i+1 / 2, j, k}, t\right)$ for the flux along $x$ direction, all the terms related with $a_{2 \mathrm{~L}}, a_{3 \mathrm{~L}}, a_{2 \mathrm{R}}, a_{3 \mathrm{R}}, \bar{a}_{1}$, and $\bar{a}_{3}$, which are related to gradients in $y$ and $z$ directions, are ignored. Consequently, Eq. (21) becomes

$$
\begin{aligned}
f\left(\boldsymbol{x}_{i+1 / 2, j, k}, t\right)= & \left\{\left[(1-\bar{A} \tau)\left(1-e^{-t / \tau}\right)+\bar{A} t\right]+\left[(t+\tau) e^{-t / \tau}-\tau\right]\right. \\
& \left.\times\left[\bar{a}_{1 \mathrm{~L}} H\left(\xi_{1}\right)+\bar{a}_{1 \mathrm{R}} H\left(-\xi_{1}\right)\right] \xi_{1}\right\} f_{0}^{(0)}+e^{-t / \tau\{\{[1} \\
& \left.\left.-(t+\tau) \xi_{1} a_{1 \mathrm{~L}}\right]-\tau A_{\mathrm{L}}\right\} H\left(\xi_{1}\right) f_{0 \mathrm{~L}}^{(0)}+\{[1-(t \\
& \left.\left.\left.+\tau) \xi_{1} a_{1 \mathrm{R}}\right]-\tau A_{\mathrm{R}}\right\} H\left(-\xi_{1}\right) f_{0 \mathrm{R}}^{(0)}\right\} .
\end{aligned}
$$

Clearly, both the Q1D-GKS and the DS-GKS only consider the normal slopes in computing the fluxes. In the Q1D-GKS, the fluxes along all directions at $t=t_{n}$ are computed independently and simultaneously according to Eq. (29), and then they are used to update flow variables simultaneously according to Eq. (28). In the DS-GKS, an operator splitting procedure is used. The fluxes are updated in an asymmetric and sequential manner, say, in the order of $x, y$, and $z$. When the fluxes along $x$ direction are obtained, it is immediately used to update all flow variables and the updated flow variables are then used to compute the fluxes along $y$ direction, which are used to update flow variables again; the twiceupdated flow variables are used to compute the fluxes along $z$ direction. Thus, the fluxes computed first depend only on the flow variables at time $t=t_{n}$; the fluxes computed next in line depend on the flow variables updated by the fluxes computed first; and the fluxes computed last depend on the flow variables updated by all the fluxes computed previously. Clearly the DS-GKS intends to utilize the fluxes as soon as they are available. This leads to an asymmetry in the flux updating, depending to the order of calculations. There is no prevailing guide to determine the order of updating in this approach.

In the compressible Navier-Stokes equations, the heat fluxes depend only on the gradients normal to cell interfaces, while the viscous fluxes depend on gradients both normal and tangential to cell interfaces. The components of the rateof-strain tensor in the $x$ direction, for instance, can be derived from the non equilibrium part in Eq. (21):

$$
\begin{gathered}
\sigma_{x x}=2 \mu \partial_{x} u+\left(\eta-\frac{2}{3} \mu\right)\left(\partial_{x} u+\partial_{y} v+\partial_{z} w\right), \\
\sigma_{x y}=\mu\left(\partial_{x} v+\partial_{y} u\right), \\
\sigma_{x z}=\mu\left(\partial_{x} w+\partial_{z} u\right),
\end{gathered}
$$

where $\eta$ is the bulk viscosity. However, in the Q1D-GKS and the DS-GKS, all the tangential derivatives are omitted as indicated by Eq. (29), which leads to

$$
\begin{gathered}
\sigma_{x x}=2 \mu \partial_{x} u+\left(\eta-\frac{2}{3} \mu\right) \partial_{x} u, \\
\sigma_{x y}=\mu \partial_{x} v, \\
\sigma_{x z}=\mu \partial_{x} w .
\end{gathered}
$$

Clearly, all tangential velocity gradients have been omitted in both the Q1D-GKS and the DS-GKS. The approximation would inevitably induce modeling errors in simulations.

We note that the existing comparative studies of the Q1D, DS, and full GKS were mostly restricted to steady laminar flows in two dimensions [37,38]. Li et al. assess the Q1D, DS, and full GKS for several laminar flows and the rarefied nonequilibrium gas flows in $2 \mathrm{D}$ and concluded that the full GKS is needed for rarefied nonequilibrium flows, and the DS-GKS is adequate for low-Reynolds-number laminar flows, while the Q1D-GKS is less so. May et al. [37] observed that the difference between the Q1D-GKS and the full GKS is small for steady subsonic laminar flows in 2D. These previous studies are inconclusive and for the most part have little bearings to the three-dimensional turbulent flows. In this work we will compare the Q1D-GKS, the DS-GKS, and the full multidimensional GKS for the DNS of fully compressible turbulent flows with shocklets in three dimensions.

\section{Simplified GKS for smooth flows}

In Eq. (21), flows are assumed to be discontinuous, and the flow variables and their gradients on both sides of a cell boundary are computed differently. The GKS is essentially a shock-capturing method, which is only first-order accurate across a shock, regardless of the order of accuracy of the method. The discontinuous treatment of hydrodynamic variables at cell boundaries will introduce numerical errors, especially for smooth flows. These errors are the consequences of inequalities of hydrodynamic variables and their gradients evaluated from both sides of a cell boundary with finite grid 
spacings. For smooth (incompressible) flows, however, hydrodynamic variables and their gradients must be continuous across a cell boundary, thus their values on both sides of a cell boundary must be equal. This simply means that $a_{1 \mathrm{~L}}$ $=a_{1 \mathrm{R}}=\bar{a}_{1 \mathrm{~L}}=\bar{a}_{1 \mathrm{R}}, a_{2 \mathrm{~L}}=a_{2 \mathrm{R}}$, and $a_{3 \mathrm{~L}}=a_{3 \mathrm{R}}$. And as a consequence, $A_{\mathrm{L}}=A_{\mathrm{R}}=\bar{A}$. Therefore, for smooth flows, Eq. (21) reduces to

$$
f\left(\boldsymbol{x}_{i+1 / 2, j, k}, t\right)=f_{0}^{(0)}[1-(\boldsymbol{a} \cdot \boldsymbol{\xi}+A) \tau+A t],
$$

where $\boldsymbol{a}:=\left(a_{1}, a_{2}, a_{3}\right)$ is given by Eq. (13a), and flow variables and their gradients at cell boundaries can be computed by using linear interpolations or other high-order reconstructions depending on accuracy requirement. This will be further discussed in Sec. II E.

Clearly, the distribution function given by Eq. (32) is much simpler than that given by Eq. (21) and hence can reduce computational time considerably. This approximation has been used to simulate low-Mach-number viscous flows or incompressible flows $[61,65,68]$. It has been observed that, for compressible decaying homogeneous turbulence, numerical schemes for smooth flows would work well, in general, if the initial turbulent Mach number $\mathrm{Ma}_{\mathrm{t}}<0.5$ [10]. Our results concur with the previous observations. This is, the approximation given by Eq. (32) works well for compressible decaying homogeneous turbulence with $\mathrm{Ma}_{\mathrm{t}}<0.6$.

In theory, the full GKS with Eq. (21) should automatically reduce to the simplified GKS with Eq. (32) in smooth flow regions when the grid spacing is infinitesimal, and the two approaches should be equivalent if the linear interpolations are used to compute both the hydrodynamic variables and their gradients at cell boundaries for both of them. However, it is not so in reality because of the difference in the numerics of these two approaches and finite grid spacings. We will compare the simplified GKS and the full GKS schemes for compressible homogeneous turbulence simulations.

\section{Viscosity, flux limiter, and artificial dissipation}

As shown in Eqs. (23) and (24), the relaxation time $\tau$ in the GKS is related to the dynamic viscosity $\mu$ and pressure $p$ by $\tau=\mu / p$. In this work, the value of the dynamic viscosity $\mu\left(\boldsymbol{x}_{i+1 / 2, j, k}\right)$ in Eq. (23) and (24) is determined by

$$
\mu=\mu_{0}\left(\frac{T}{T_{0}}\right)^{0.76}
$$

where $\mu_{0}$ and $T_{0}$ are material-dependent constants. In Eq. (24), we use $\tau_{\mu}=\mu / p=\mu \beta / \rho$ to calculate $\tau_{\mu}$, and the value of $\tau_{\mu}\left(\boldsymbol{x}_{i+1 / 2, j, k}\right)$ at $t=t_{n}$ is determined by the values of $T\left(\boldsymbol{x}_{i+1 / 2, j, k}\right)$ and $\rho\left(\boldsymbol{x}_{i+1 / 2, j, k}\right)$ at the previous time step $t=t_{n-1}$, given by the hydrodynamic variables $\mathbf{h}\left(\boldsymbol{x}_{i+1 / 2, j, k}, t_{n-1}\right)$ through the conserved variables $\mathbf{W}\left(\boldsymbol{x}_{i+1 / 2, j, k}\right)$ of Eq. (19).

The term $\sigma \tau_{\sigma}$ in Eq. (24) gives rise to artificial dissipation, where $\tau_{\sigma}$ is the relaxation time corresponding to the artificial dissipation. The parameter $\sigma \in[0,1]$ is used to adjust the intensity of artificial dissipation. We should emphasize that the artificial dissipation is necessary only when the Mach number is sufficiently high. When the turbulence Mach number is greater than 0.65 or so, we must use a lim- iter to stabilize the code. However, when the turbulence Mach number is further increased beyond a certain point, we must use artificial dissipation in addition to a limiter. We will assess the effect of the artificial dissipation on turbulence.

The values of pressure evaluated at the left and the right of the cell boundary $\boldsymbol{x}_{i+1 / 2, j, k}, p_{\mathrm{L}}$ and $p_{\mathrm{R}}$, are obtained from $\mathbf{h}\left(\boldsymbol{x}_{i+1 / 2, j, k}^{-}\right)$and $\mathbf{h}\left(\boldsymbol{x}_{i+1 / 2, j, k}^{+}\right)$, respectively. Therefore, the artificial dissipation is effective only when shocks are treated as discontinuities. Obviously, when flow fields are continuous, $p_{\mathrm{L}}=p_{\mathrm{R}}$, hence the artificial dissipation vanishes. We use the van Leer limiter $[31,66]$ in the interpolations of hydrodynamic variables at cell interfaces, which also introduces numerical dissipations. These dissipations due to the limiter and the artificial relaxation time $\tau_{\sigma}$ are the so-called dynamic artificial dissipations [31]. On the other hand, the GKS also assumes discontinuity at cell interfaces in the reconstruction step. The averaging process in the initial reconstruction also introduces numerical dissipations, which are the so-called kinematic artificial dissipations [31]. All artificial dissipations, whether dynamic or kinematic, can severely affect the accuracy of the GKS scheme although they can enhance numerical stability. We will assess the effects of the artificial dissipations due to the flux limiter and initial reconstruction in this work.

\section{E. Interpolations at cell boundaries for smooth flows}

For smooth flows, the flow variables and their gradients at cell boundaries are obtained by interpolations. To achieve a second-order accuracy, it is sufficient to the following linear interpolations, for instance, in the $x$ direction,

$$
\mathbf{W}_{i+1 / 2, j, k}=\frac{1}{2}\left(\mathbf{W}_{i, j, k}+\mathbf{W}_{i+1, j, k}\right)
$$

$$
\partial_{x} \mathbf{W}_{i+1 / 2, j, k}=\frac{1}{\Delta x}\left(\mathbf{W}_{i+1, j, k}-\mathbf{W}_{i, j, k}\right),
$$

$$
\begin{aligned}
& \partial_{y} \mathbf{W}_{i+1 / 2, j, k}=\frac{1}{2 \Delta y}\left(\mathbf{W}_{i+1 / 2, j+1, k}-\mathbf{W}_{i+1 / 2, j-1, k}\right), \\
& \partial_{z} \mathbf{W}_{i+1 / 2, j, k}=\frac{1}{2 \Delta z}\left(\mathbf{W}_{i+1 / 2, j, k+1}-\mathbf{W}_{i+1 / 2, j, k-1}\right) .
\end{aligned}
$$

Unless otherwise stated, the above linear interpolations will be used in the simplified GKS. It should also be noted that, when the linear interpolation of Eq. (34a) is also used in the reconstruction for the full GKS, the gradients given above are fully equivalent to those given by Eqs. (16) and (18) for smooth flows. To understand the effect due to the interpolations, we will also test the following third-order interpolations in our simulations,

$$
\mathbf{W}_{i+1 / 2, j, k}=\frac{9}{16}\left(\mathbf{W}_{i, j, k}+\mathbf{W}_{i+1, j, k}\right)-\frac{1}{16}\left(\mathbf{W}_{i-1, j, k}+\mathbf{W}_{i+2, j, k}\right),
$$




$$
\begin{aligned}
\partial_{x} \mathbf{W}_{i+1 / 2, j, k}= & \frac{5}{4 \Delta x}\left(\mathbf{W}_{i+1, j, k}-\mathbf{W}_{i, j, k}\right)-\frac{1}{12 \Delta x}\left(\mathbf{W}_{i+2, j, k}\right. \\
- & \left.\mathbf{W}_{i-1, j, k}\right) \\
\partial_{y} \mathbf{W}_{i+1 / 2, j, k}= & \frac{8}{12 \Delta y}\left(\mathbf{W}_{i+1 / 2, j+1, k}-\mathbf{W}_{i+1 / 2, j-1, k}\right) \\
& -\frac{1}{12 \Delta y}\left(\mathbf{W}_{i+1 / 2, j+2, k}-\mathbf{W}_{i+1 / 2, j-2, k}\right), \\
\partial_{z} \mathbf{W}_{i+1 / 2, j, k}= & \frac{8}{12 \Delta z}\left(\mathbf{W}_{i+1 / 2, j, k+1}-\mathbf{W}_{i+1 / 2, j, k-1}\right) \\
& -\frac{1}{12 \Delta z}\left(\mathbf{W}_{i+1 / 2, j, k+2}-\mathbf{W}_{i+1 / 2, j, k-2}\right)
\end{aligned}
$$

Note that, in calculating the gradients at the cell interface $x_{i+1 / 2}$ using above interpolations given by Eqs. (34c) and (34d) or Eqs. (35c) and (35d), all the values of the flow variables at $x_{i+1 / 2}$ must be interpolated from the cell center values using Eq. (34a) or Eq. (35a), respectively. Obviously, interpolations for $y$ and $z$ directions can be easily done, in a similar manner as Eq. (34) or Eq. (35).

For smooth flows, the linear interpolations given by Eq. (34) make the GKS scheme a second-order accurate one $[34,38,40]$. For DNS of turbulence, however, quantities related to high-order gradients of flow variables may be sensitive to the accuracy of interpolations used at cell interfaces, thus higher-order interpolations may be required. We will investigate the effects due to different interpolations at cell interfaces for DNS of compressible homogeneous turbulence.

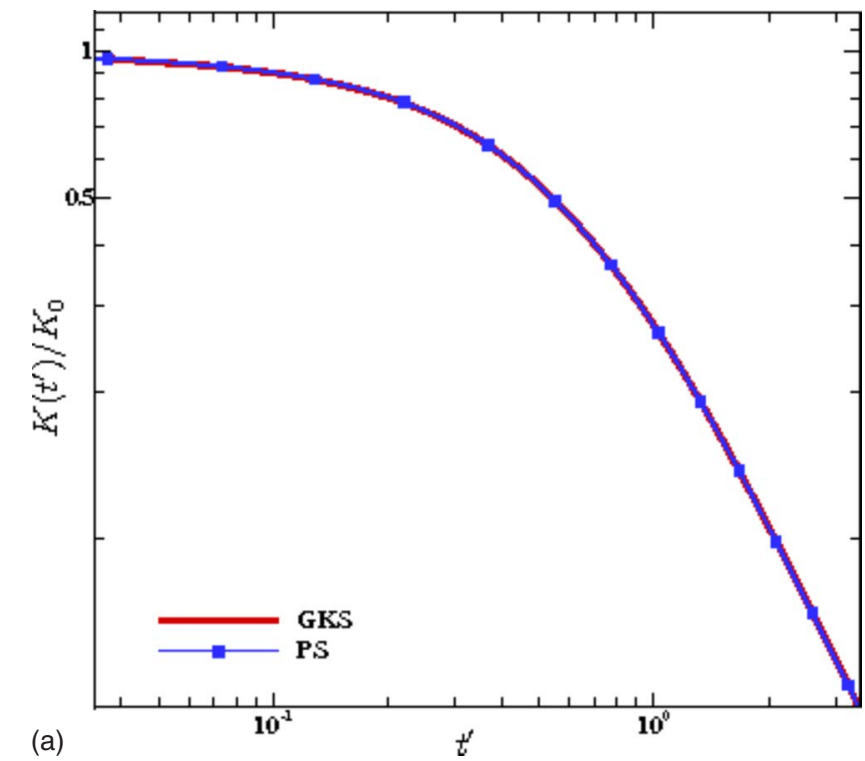

\section{F. Bulk viscosity $\eta$}

For thermochemical nonequilibrium hypersonic flows, the internal degrees of freedom of gas molecules must be considered. In the framework of continuum theory and the Navier-Stokes equations, the internal degrees of freedom is accounted for through the bulk (second) viscosity

$$
\eta=\frac{2 Z}{3(Z+3)} \mu,
$$

where $\mu$ is the dynamic (first) viscosity and $Z$ is the number of the internal degrees of freedom, which is equal to 2 for diatomic gases with rotational degrees of freedom in threedimensional space. Thus $\eta=4 \mu / 15$ with $Z=2$. In the GKS, the bulk viscosity $\eta$ can be easily adjusted by tuning the parameter $Z$. We will study the effects of the bulk viscosity $\eta$, and in turn the compressibility $\boldsymbol{\nabla} \cdot \boldsymbol{u}$, in DNS of compressible homogeneous turbulence.

\section{COMPRESSIBLE DECAYING HOMOGENEOUS ISOTROPIC TURBULENCE}

\section{A. Governing equations and flow conditions}

We use the GKS method to solve the fully compressible Navier-Stokes equations in $3 \mathrm{D}$,

$$
\begin{gathered}
\partial_{t} \rho+\nabla \cdot \rho \boldsymbol{u}=0, \\
\partial_{t} \rho \boldsymbol{u}+\nabla \cdot \rho \boldsymbol{u} \boldsymbol{u}+\boldsymbol{\nabla} p=\boldsymbol{\nabla} \cdot \boldsymbol{\sigma}, \\
\partial_{t} E+\nabla \cdot E \boldsymbol{u}+\boldsymbol{\nabla} \cdot p \boldsymbol{u}=\frac{1}{\alpha} \boldsymbol{\nabla} \cdot(\kappa \boldsymbol{\nabla} T)+\boldsymbol{\nabla} \cdot(\boldsymbol{\sigma} \cdot \boldsymbol{u}), \\
\sigma_{i j}:=\mu\left(\partial_{i} u_{j}+\partial_{j} u_{i}\right)+\left(\eta-\frac{2}{3} \mu\right) \delta_{i j} \boldsymbol{\nabla} \cdot \boldsymbol{u},
\end{gathered}
$$

where $\boldsymbol{\sigma}$ is the stress tensor and $\kappa$ is the heat conductivity. The dimensionless parameters for the compressible NavierStokes equations are

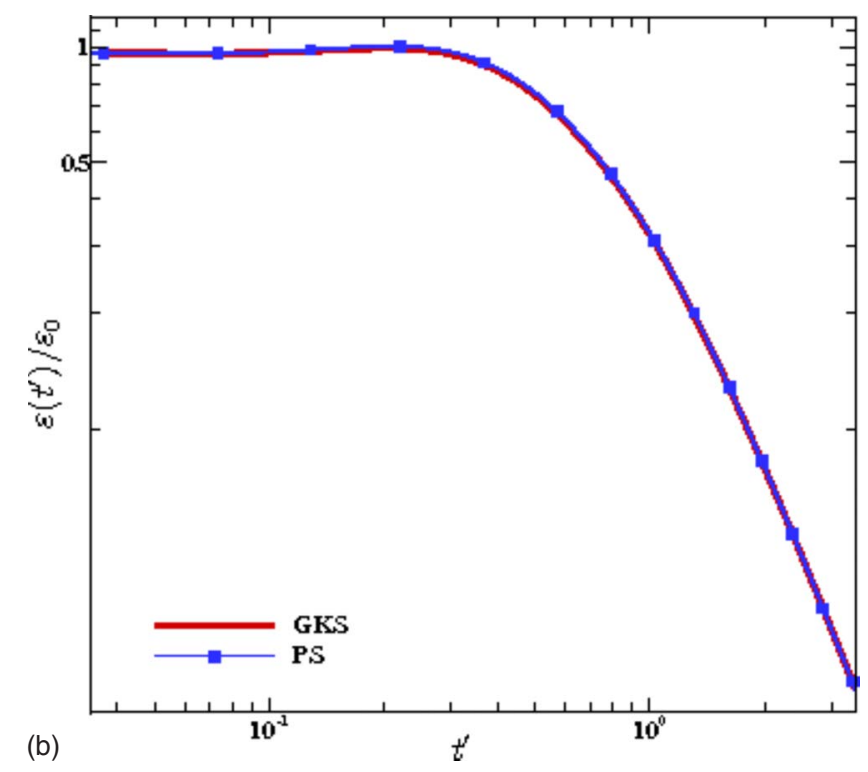

FIG. 2. (Color online) The kinetic energy $K\left(t^{\prime}\right) / K_{0}$ and dissipation rate $\varepsilon\left(t^{\prime}\right) / \varepsilon_{0}$ in DHIT. $\mathrm{Ma}_{\mathrm{t}}=0.1, \operatorname{Re}_{\lambda}=24.0$, and $N^{3}=256^{3}$. The GKS method (thick lines) vs pseudospectral method (thin lines with symbols). 


$$
\operatorname{Re}=\frac{\rho_{0} c_{s 0} L}{\mu_{0}}, \quad \operatorname{Ma}=\frac{U}{c_{s 0}}, \quad c_{s 0}=\sqrt{\gamma R T_{0}}, \quad \operatorname{Pr}=\frac{\mu_{0} c_{p}}{\kappa_{0}}=0.7 .
$$

For decaying homogeneous isotropic turbulence (DHIT), the flow domain is a three-dimensional cube of size $L^{3}=(2 \pi)^{3}$ with periodic boundary conditions in all three directions. The cube is discretized with a uniform Cartesian mesh size $N^{3}$. A divergence-free random initial velocity field $\boldsymbol{u}_{0}(\boldsymbol{x})$ is generated for a given spectrum by using the method of Rogallo [69] with a specified root mean square $(\mathrm{rms})$ :

$$
u^{\prime}:=\frac{1}{\sqrt{3}} \sqrt{\langle\boldsymbol{u} \cdot \boldsymbol{u}\rangle} .
$$

The initial energy spectrum $\widetilde{E}_{0}(k)$ in the Fourier space $\boldsymbol{k}$ is given by

$$
\widetilde{E}_{0}(k)=A_{0} k^{4} \exp \left(-2 k^{2} / k_{0}^{2}\right),
$$

where $A_{0}=1.3 \times 10^{-4}$ and $k_{0}=8$. At $t=0$,

$$
\begin{gathered}
K_{0}=\frac{3 A_{0}}{64} \sqrt{2 \pi} k_{0}^{5}, \\
\Omega_{0}=\frac{15 A_{0}}{256} \sqrt{2 \pi} k_{0}^{7}, \\
\varepsilon_{0}=2 \frac{\mu_{0}}{\rho_{0}} \Omega_{0},
\end{gathered}
$$$$
\operatorname{Re}_{\lambda}:=\frac{\langle\rho\rangle u^{\prime} \lambda}{\langle\mu\rangle}=\frac{(2 \pi)^{1 / 4}}{4} \frac{\rho_{0}}{\mu_{0}} \sqrt{2 A_{0}} k_{0}^{3 / 2},
$$

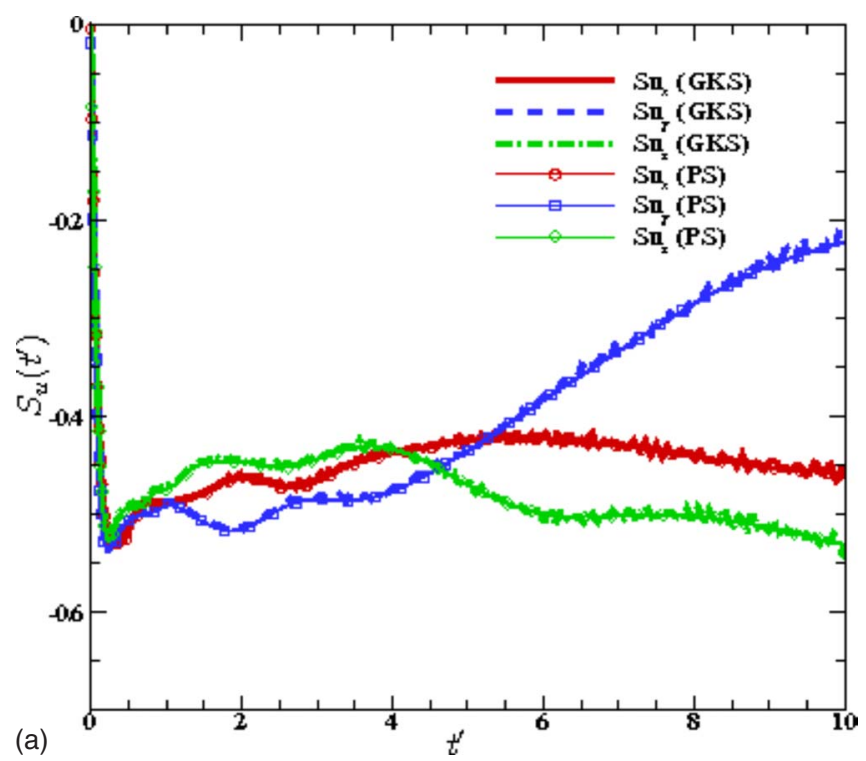

$$
\mathrm{Ma}_{\mathrm{t}}:=\frac{\sqrt{3} u^{\prime}}{\left\langle c_{s}\right\rangle}=\frac{\sqrt{3} u^{\prime}}{\sqrt{\gamma R T_{0}}},
$$

where $K_{0}, \Omega_{0}$, and $\varepsilon_{0}$ are the initial kinetic energy, enthalpy, and dissipation rate, respectively; and $\mathrm{Re}_{\lambda}$ and $\mathrm{Ma}_{\mathrm{t}}$ are the initial Taylor microscale Reynolds number and turbulence Mach number, respectively. With $u^{\prime}, \operatorname{Re}_{\lambda}$, and $\mathrm{Ma}_{\mathrm{t}}$ given at $t=0$, we set $\rho_{0}=1$, and determine $\mu_{0}$ and $T_{0}$ from $\operatorname{Re}_{\lambda}$ and $\mathrm{Ma}_{\mathrm{t}}$, respectively.

The following quantities of turbulence will be computed in our simulations $[5,7,8,10,70]$ :

$$
\begin{gathered}
K(t):=\frac{1}{2}\langle\rho \boldsymbol{u} \cdot \boldsymbol{u}\rangle, \\
\varepsilon(t):=2\left\langle\frac{\mu}{\rho} \boldsymbol{u} \cdot \nabla^{2} \boldsymbol{u}\right\rangle, \\
S_{u}(t)=\frac{1}{3} \sum_{i} S_{u_{i}}, \\
S_{u_{i}}=\frac{\left\langle\left(\partial_{i} u_{i}\right)^{3}\right\rangle}{\left\langle\left(\partial_{i} u_{i}\right)^{2}\right\rangle^{3 / 2}}, \\
F_{u}(t)=\frac{1}{3} \sum_{i} F_{u_{i}}, \\
F_{u_{i}}(t)=\frac{\left\langle\left(\partial_{i} u_{i}\right)^{4}\right\rangle}{\left\langle\left(\partial_{i} u_{i}\right)^{2}\right\rangle^{2}},
\end{gathered}
$$

where $K(t)$ and $\varepsilon(t)$ are the kinetic energy and dissipation rate, respectively; $S_{u_{i}}$ and $F_{u_{i}}$ are the skewness and flatness of the velocity derivative $\partial_{i} u_{i}$, with $i \in\{x, y, z\}$, and $S_{u}$ and $F_{u}$ are the skewness and flatness averaged over three directions,

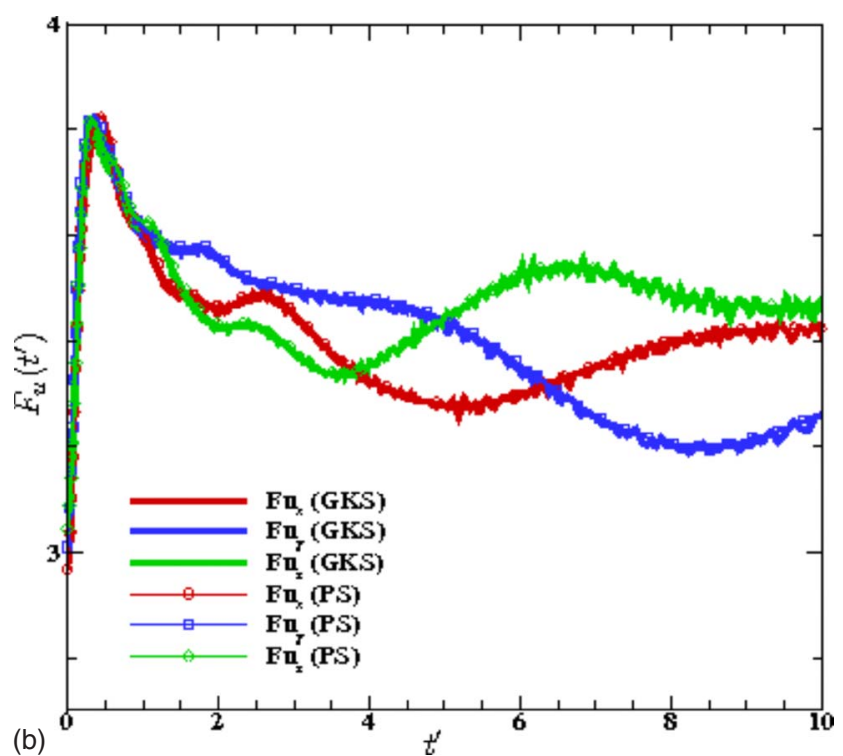

FIG. 3. (Color online) The skewness $S_{u_{i}}\left(t^{\prime}\right)$ (left) and flatness $F_{u_{i}}\left(t^{\prime}\right)$ (right) in DHIT. $\mathrm{Ma}_{\mathrm{t}}=0.1, \operatorname{Re}_{\lambda}=24.0$, and $N^{3}=256^{3}$. The GKS method (lines) vs pseudospectral method (symbols). 

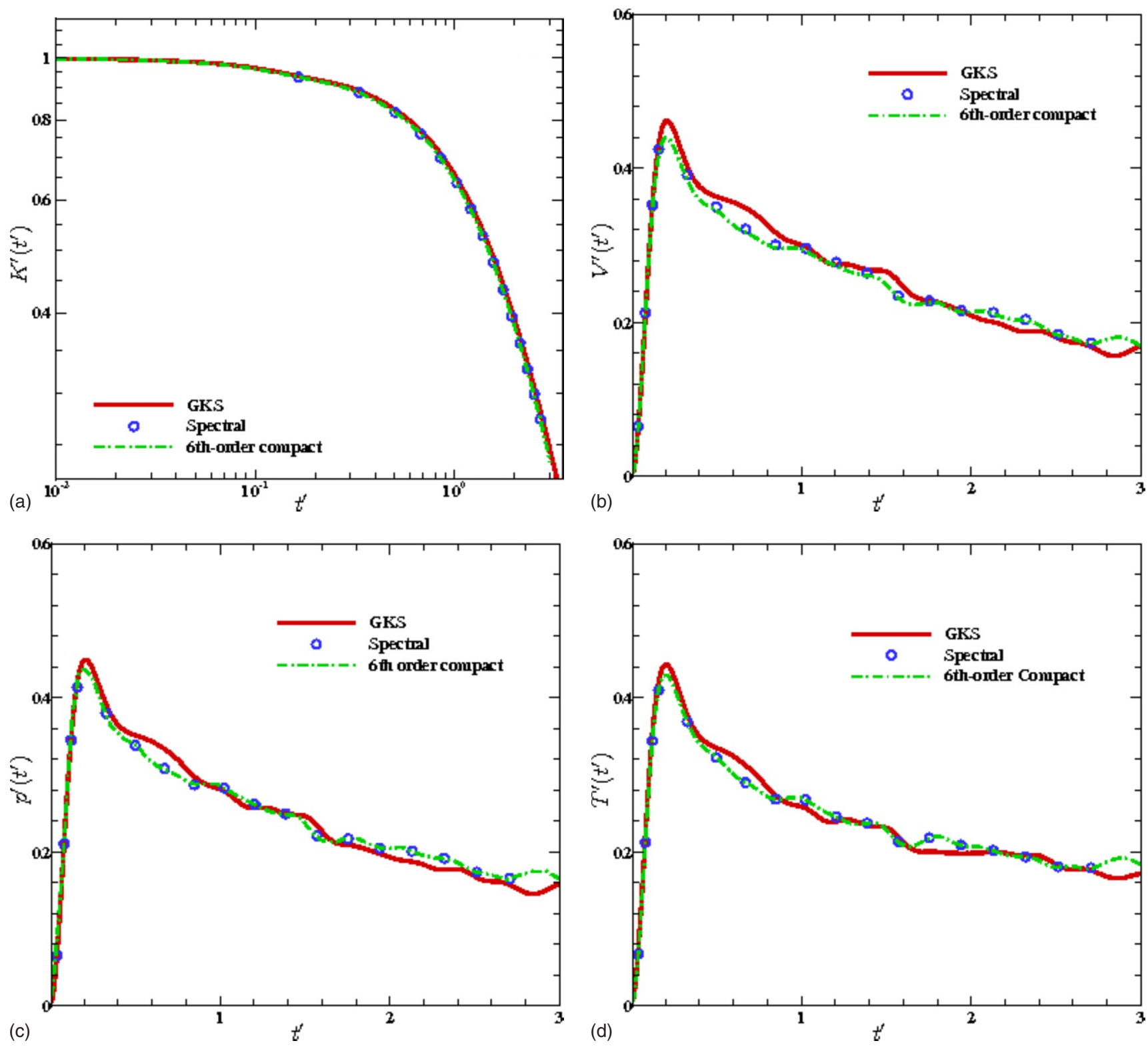

FIG. 4. (Color online) The comparison of the GKS and the dealiased spectral computation [13] on the kinetic energy and thermodynamic fluctuations at $\mathrm{Ma}_{\mathrm{t}}=0.3, \mathrm{Re}_{\lambda}=30.0$ and $N^{3}=64^{3}$ : (a) kinetic energy $K^{\prime}\left(t^{\prime}\right)$, (b) the rms of the specific volume $V^{\prime}\left(t^{\prime}\right)$, (c) the rms of the pressure $p^{\prime}\left(t^{\prime}\right)$, and (d) the rms of the temperature $T^{\prime}\left(t^{\prime}\right)$.

respectively. We will investigate effects on these quantities due to numerics.

\section{B. Code validation}

To validate our code, we first test the code for the incompressible DHIT by using a low turbulent Mach number $\mathrm{Ma}_{\mathrm{t}}=0.1$ and compare the results with a pseudospectral (PS) method. For the pseudospectral method we use here, the second-order Adam-Bashforth scheme is used to numerically integrate the nonlinear term, while the viscous term is treated exactly. In the GKS method, no artificial dissipation is used in this work unless otherwise stated, that is, we set $\sigma=0$ in Eq. (24). The mesh size used for the validation is $N^{3}=256^{3}$ and the Taylor Reynolds number is set to $\operatorname{Re}_{\lambda}=24$.0. With the resolution given, the flow is well resolved. In this test, the DHIT is simulated by using the simplified GKS with the third-order interpolations given by Eq. (35) and the bulk viscosity $\eta=4 \mu / 15[Z=2$ in Eq. (36)].

We first compare the kinetic energy $K(t)$ and the dissipation rate $\varepsilon(t)$ computed by using the GKS method and the pseudospectral method in Fig. 2. The time is normalized by the turbulence turnover time $\tau_{0}=K_{0} / \varepsilon_{0}$, i.e., $t^{\prime}=t / \tau_{0}$, and the simulations are carried out to $t^{\prime} \approx 3.5$. We observe excellent agreement between the results obtained by both methods.

We next show in Fig. 3 the comparison of the skewness $S_{u_{i}}$ and the flatness $F_{u_{i}}, i \in\{x, y, z\}$, obtained by using the two methods. The skewness and flatness are related to the fourthorder and the first-order velocity gradients, respectively, and are sensitive to numerical accuracy and dissipations. It can 

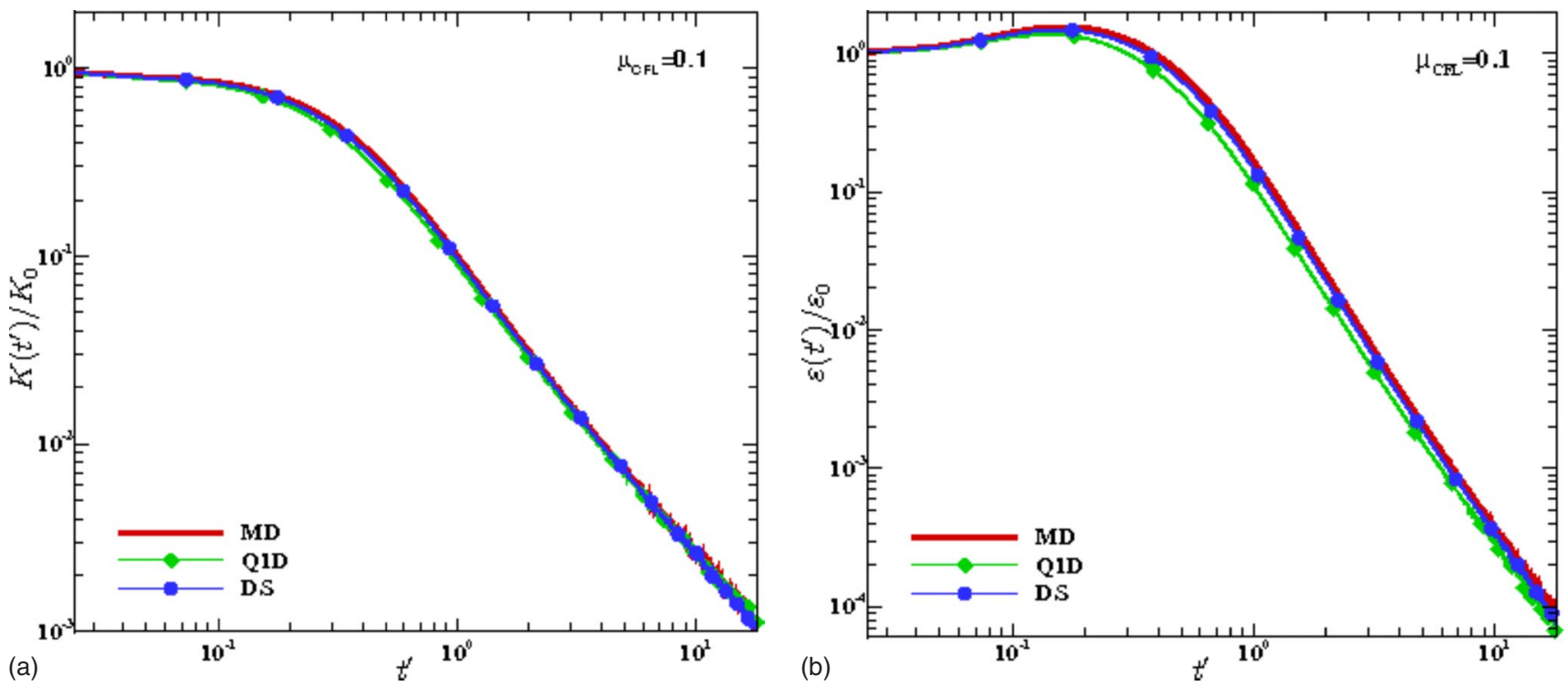

FIG. 5. (Color online) The evolution of the kinetic energy $K\left(t^{\prime}\right) / K_{0}$ (left) and dissipation rate $\varepsilon(t) / \varepsilon_{0}$ (right) with $\mathrm{Ma}_{\mathrm{t}}=0.5, \mathrm{Re}_{\lambda}=72.0$, $N^{3}=128^{3}$, and $\mu_{\mathrm{CFL}}=0.1$. The results are computed by using the full MD GKS, the Q1D GKS, and the DS-GKS.

be a challenging task for a second-order method, such as the GKS method, to accurately compute these quantities. The results of Fig. 3 show that the skewness and flatness computed from the GKS method agree very well with those from the pseudospectral method, and they are close to the theoretical values for isotropic turbulence, $S_{u} \approx-0.5$ and $F_{u} \approx 3.5$.

To further validate the GKS code, we use the same GKS strategy for a compressible DHIT with $\mathrm{Ma}_{\mathrm{t}}=0.3$ and $\mathrm{Re}_{\lambda}$ $=30$ and compare our results with the data obtained with a dealiased spectral method [13]. The mesh size is $N^{3}=64^{3}$, the same as what has been used previously $[13,14]$. In this case, a divergence-free random initial velocity field $\boldsymbol{u}_{0}$ is generated with $A_{0}=3.74 \times 10^{-4}$ and $k_{0}=4$ for the initial energy spectrum given by Eq. (40).

We compute the evolution of the normalized kinetic energy $K^{\prime}\left(t^{\prime}\right)$, the normalized root mean squares of the pressure fluctuation, $p^{\prime}\left(t^{\prime}\right)$, the temperature fluctuation, $T^{\prime}\left(t^{\prime}\right)$, and the specific volume fluctuation, $V^{\prime}\left(t^{\prime}\right)$,

$$
\begin{gathered}
K^{\prime}:=\frac{3 u^{\prime 2}}{c_{s 0}^{2} \mathrm{Ma}_{\mathrm{t}}^{2}}, \\
p^{\prime}:=\frac{\left\langle(p-\bar{p})^{2}\right\rangle^{1 / 2}}{\gamma p_{0} \mathrm{Ma}_{\mathrm{t}}^{2}}, \\
T^{\prime}:=\frac{\left\langle(T-\bar{T})^{2}\right\rangle^{1 / 2}}{(\gamma-1) T_{0} \mathrm{Ma}_{\mathrm{t}}^{2}}, \\
V^{\prime}:=\frac{\left\langle(V-\bar{V})^{2}\right\rangle^{1 / 2}}{V_{0} \mathrm{Ma}_{\mathrm{t}}^{2}},
\end{gathered}
$$

where $V=1 / \rho, V_{0}=1 / \rho_{0}$, and $\bar{V}=1 / \bar{\rho} ; p_{0}, T_{0}$, and $\rho_{0}$ are the initial mean values of $p, T$, and $\rho$, respectively; $\bar{p}, \bar{T}$, and $\bar{\rho}$ are the instantaneous mean values of $p, T$, and $\rho$, respectively; and $c_{s 0}=\sqrt{\gamma R T_{0}}$. The results of $K^{\prime}\left(t^{\prime}\right), V^{\prime}\left(t^{\prime}\right), p^{\prime}\left(t^{\prime}\right)$, and $T^{\prime}\left(t^{\prime}\right)$ are shown in Fig. 4, and our results agree well with the existing data obtained by using spectral and highorder finite difference methods [13]. We should also note that, with a small mesh size of $N^{3}=64^{3}$, the initial conditions have observable effects on the results of $K^{\prime}\left(t^{\prime}\right), V^{\prime}\left(t^{\prime}\right)$, $p^{\prime}\left(t^{\prime}\right)$, and $T^{\prime}\left(t^{\prime}\right)$. This is responsible in part for the differences between our results and the existing data shown in Fig. 4.

\section{NUMERICAL RESULTS AND DISCUSSIONS}

The main focus in this study is to investigate the efficacy and fidelity of the GKS schemes for direct numerical simulations (DNSs) of compressible homogeneous turbulence. Thus turbulence physics is not main focus of this study. In what follows, we will investigate the effects on interested turbulence quantities due to approximations in the flux construction, flux limiters, interpolations at cell boundaries, and the bulk viscosity under different flow conditions. We do not use any artificial dissipation in the results presented in this section unless otherwise stated; that is, we set $\sigma=0$ in Eq. (24). Unless otherwise stated, we will use the linear interpolations of Eqs. (16) and (18) at cell boundaries, and consider the bulk viscosity $\eta=4 \mu / 15$.

For the cases present in this section, we will use the resolution of $N^{3}=128^{3}$ and the Taylor microscale Reynolds number $\operatorname{Re}_{\lambda}=72.0$, which has been used previously [10]. The initial turbulence Mach number $\mathrm{Ma}_{\mathrm{t}}$ will be between 0.1 and 0.6 .

\section{A. Effect of multidimensional fluxes}

We will first assess the necessity to use the full multidimensional (MD GKS) fluxes based on Eq. (21), as oppose to the Q1D and DS fluxes based on Eq. (29) for DNS of compressible DHIT. The mesh size we use is $N^{3}=128^{3}$, and the flow conditions are $\mathrm{Ma}_{\mathrm{t}}=0.5$ and $\mathrm{Re}_{\lambda}=72.0$. We also use 

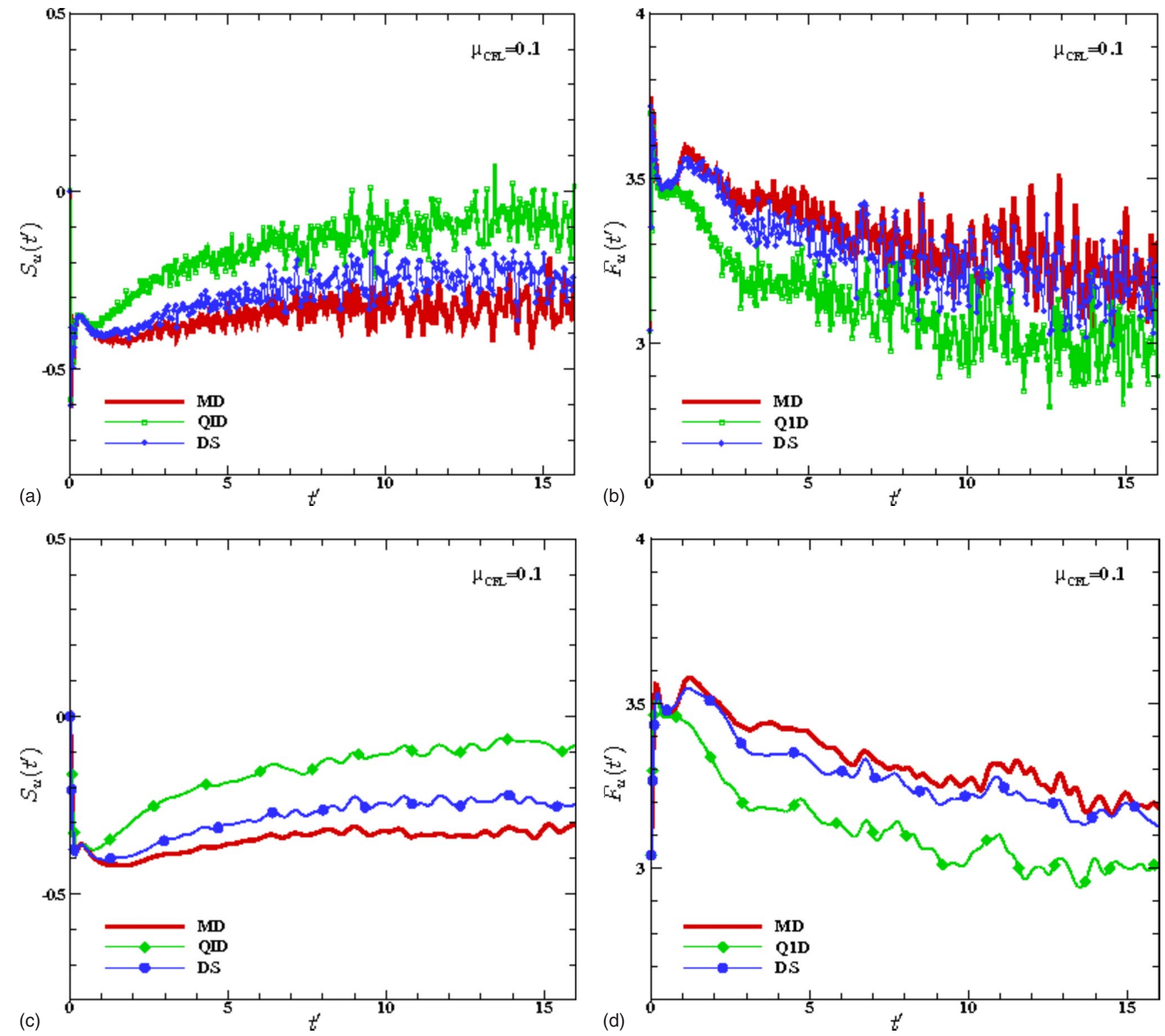

FIG. 6. (Color online) The evolution of the skewness $S_{u}\left(t^{\prime}\right)$ (left) and flatness $F_{u}\left(t^{\prime}\right)$ (right) with $\mathrm{Ma}_{\mathrm{t}}=0.5, \operatorname{Re}_{\lambda}=72.0, N^{3}=128^{3}$, and $\mu_{\mathrm{CFL}}=0.1$. The results are computed by using the full MD GKS, the Q1D-GKS, and the DS-GKS. In the bottom row, the results are smoothed.

different values of the CFL number $\mu_{\mathrm{CFL}}$ to test the numerical stability of these GKS schemes.

We first show in Fig. 5 the kinetic energy $K\left(t^{\prime}\right) / K_{0}$ and the dissipation rate $\varepsilon\left(t^{\prime}\right) / \varepsilon_{0}$ computed from three GKS schemes, denoted as MD, Q1D, and DS GKS schemes. The CFL number is $\mu_{\mathrm{CFL}}=0.1$. While the results of $K\left(t^{\prime}\right) / K_{0}$ computed from three GKS schemes are rather close to each other on the log-log scales, a close look reveals that during the initial stage $t^{\prime}<1.0$ the kinetic energy $K\left(t^{\prime}\right) / K_{0}$ computed from the full MD GKS is greater than that from the DS GKS scheme, which is greater than that from the Q1D GKS scheme. This suggests that both the DS and Q1D-GKS schemes are more dissipative then the full multidimensional GKS scheme and especially so is the Q1D-GKS scheme although all these schemes are all second-order accurate. This fact is further confirmed by the results for the dissipation rate $\varepsilon\left(t^{\prime}\right) / \varepsilon_{0}$. As clearly shown in the figure, the maximum of $\varepsilon\left(t^{\prime}\right) / \varepsilon_{0}$ computed from the full MD GKS is greater than that from the DS and Q1D-GKS schemes and in that order. This is because the numerical dissipations in DS and Q1D-GKS schemes weaken the nonlinearity in the Navier-Stokes equation and, in turn, the peak of the dissipation rate. This also suggests that numerical dissipations can effectively decrease the Reynolds number of the flow.

We next show in Fig. 6 the skewness $S_{u}\left(t^{\prime}\right)$ and the flatness $F_{u}\left(t^{\prime}\right)$ computed from three GKS schemes. Because the skewness and flatness are related to fourth- and first-order velocity derivatives, respectively, both these quantities can distinguish the three schemes more prominently. For $S_{u}\left(t^{\prime}\right)$, during a short initial period of time $t^{\prime}<0.8$, the results computed from GKS schemes agree well with each other. After this short initial period of time, the result of the Q1D-GKS 

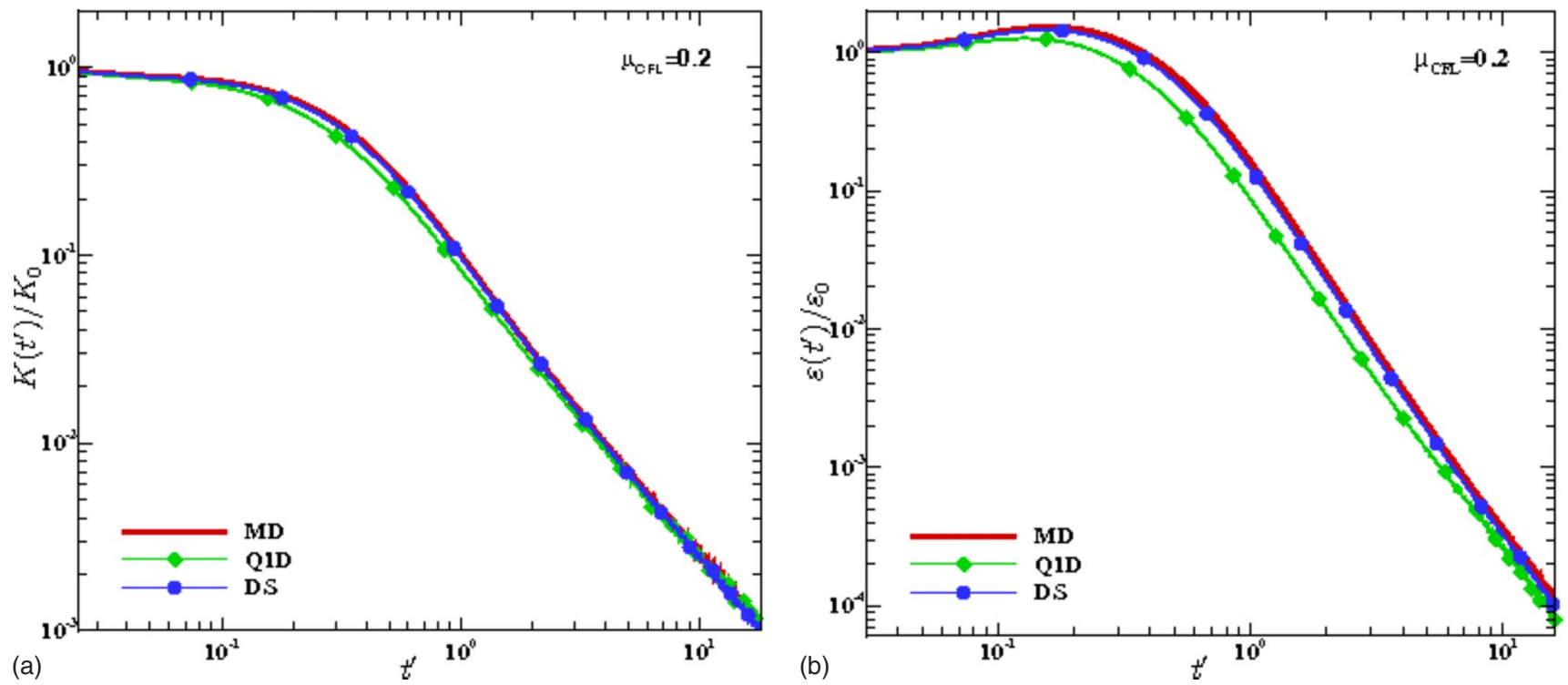

FIG. 7. (Color online) The evolution of the kinetic energy $K\left(t^{\prime}\right) / K_{0}$ (left) and dissipation rate $\varepsilon(t) / \varepsilon_{0}$ (right). Similar to Fig. 5, with $\mu_{\mathrm{CFL}}=0.2$.

quickly and significantly deviates from the results of the full MD GKS and the DS-GKS, which agree with each other closely for the entire period of the simulation $t^{\prime} \approx 16.0$ and remain close to the theoretical value of $S_{u} \approx-0.5$. Similar observations can be made for the flatness $F_{u}\left(t^{\prime}\right)$ as well. The results obtained by all three GKS schemes agree with each other for a very short period of time initially. After this short initial period of time, the result of $F_{u}\left(t^{\prime}\right)$ computed from the Q1D-GKS quickly deviates from that computed by using the full MD GKS and the DS-GKS, while the result computed by using the DS-GKS starts to deviate significantly from the MD-GKS result only after $t^{\prime}>8.0$. The MD-GKS result maintains close to the theoretical value of $F_{u} \approx 3.5$.

To investigate the effect of the CFL number $\mu_{\mathrm{CFL}}$, we repeat the simulations with $\mu_{\mathrm{CFL}}=0.2$ and 0.5 . The evolutions of the kinetic energy, $K\left(t^{\prime}\right) / K_{0}$, and the dissipation rate, $\varepsilon(t) / \varepsilon_{0}$ for $\mu_{\mathrm{CFL}}=0.2$ are shown in Fig. 7 . The differences between the Q1D-GKS results for both $K\left(t^{\prime}\right) / K_{0}$ and $\varepsilon(t) / \varepsilon_{0}$ and those computed by using the DS-GKS and the full MD GKS are obviously larger than the case of $\mu_{\mathrm{CFL}}=0.1$ of Fig. 5. Similar observations can be made for the skewness $S_{u}\left(t^{\prime}\right)$ and flatness $F_{u}\left(t^{\prime}\right)$ shown in Fig. 8. We also observe that the differences between results computed by using the DS-GKS and the full MD GKS appear to be affected very little by the CFL number $\mu_{\mathrm{CFL}}$.

As the CFL number is increased to $\mu_{\mathrm{CFL}}=0.5$, the Q1DGKS becomes unstable, as shown in Fig. 9 for $K\left(t^{\prime}\right) / K_{0}$ and $\varepsilon(t) / \varepsilon_{0}$. In addition, the differences for the results of the skewness $S_{u}\left(t^{\prime}\right)$ and flatness $F_{u}\left(t^{\prime}\right)$ (Fig. 10) computed by using the DS-GKS and the full MD GKS are further amplified, and the values of $S_{u}\left(t^{\prime}\right)$ and $F_{u}\left(t^{\prime}\right)$ computed by using the full MD GKS remain close to their theoretical values. The high-frequency oscillations in both $S_{u}\left(t^{\prime}\right)$ and $F_{u}\left(t^{\prime}\right)$ weaken as the CFL number $\mu_{\mathrm{CFL}}$ increases. This is understandable because as the CFL number $\mu_{\mathrm{CFL}}$ increases so do the time step size and the corresponding truncation errors. The former reduces the resolution in time, and the latter in- creases numerical dissipations; both effects suppress the high-frequency oscillations.

Our results show that among three schemes, the Q1DGKS is the most dissipative, least accurate, and most unstable. The full MD GKS is the best one in terms of numerical dissipation, accuracy, and stability, while the DS-GKS ranks second. Obviously, the Q1D-GKS is not adequate for DNS of homogeneous turbulence flows, while the DS-GKS is adequate for the purpose provided that the CFL number is small enough. We also observe that an increase in numerical dissipations leads to an increase in the skewness $S_{u}\left(t^{\prime}\right)$ as well as a decrease in the flatness $F_{u}\left(t^{\prime}\right)$ after a very short initial period in time.

We also assess the computational efficiencies of the three approaches. For the part related to the calculation of fluxes, the computational effort of the DS-GKS is only slightly more than that of the Q1D-GKS (less than 5\%), while the computational effort of the full GKS is about twice of that of the DS-GKS. In light of the results above and the consideration of the computational efficiency, the DS-GKS appears to be adequate to compute low-order turbulence statistics within the parameter ranges tested for the DNS for decaying turbulence.

\section{B. Simplified GKS vs full multidimensional GKS}

The simplified GKS with the fluxes determined by Eq. (32) is much simpler, thus much computationally efficient than the full multidimensional GKS with the fluxes determined by Eq. (21). Another benefit of using the simplified GKS for smooth flows is numerical consistency of the values of hydrodynamic variables and their gradients at cell boundaries because the simplified GKS assumes these quantities to be continuous, while the multidimensional GKS assumes them to be discontinuous. It is important to note that the simplified GKS is a multidimensional scheme, it does not neglect the derivatives tangential to cell interfaces. We will 

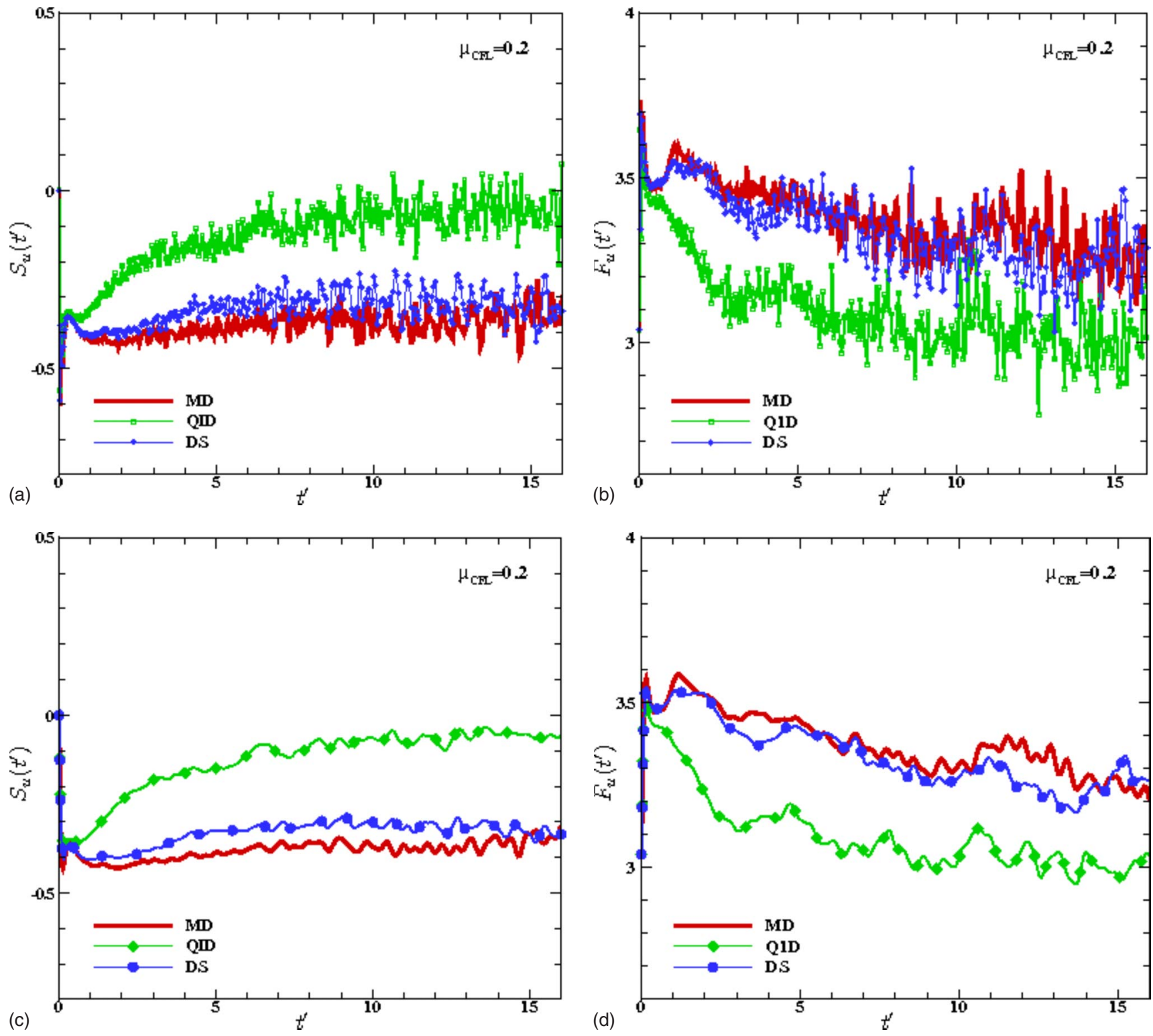

FIG. 8. (Color online) The evolution of the skewness $S_{u}\left(t^{\prime}\right)$ (left) and flatness $F_{u}\left(t^{\prime}\right)$ (right). Similar to Fig. 6, with $\mu_{\mathrm{CFL}}=0.2$. In the bottom row, the results are smoothed.

compare the simplified GKS and the multidimensional GKS for the DNS of compressible DHIT. For both schemes, the linear interpolations of Eq. (34) are used to compute the conservative variables and their gradients at cell boundaries.

We first compare the results of the simplified GKS and the full GKS for the following flow conditions: $\mathrm{Ma}_{\mathrm{t}}=0.1, \mathrm{Re}_{\lambda}$ $=72.0, \mu_{\mathrm{CFL}}=0.2$, and with a mesh size of $N^{3}=128^{3}$. Figure 11 shows the evolutions of the kinetic energy $K\left(t^{\prime}\right) / K_{0}$ and the dissipation rate $\varepsilon\left(t^{\prime}\right) / \varepsilon_{0}$, and Fig. 12 shows that of the skewness $S_{u}\left(t^{\prime}\right)$ and the flatness $F_{u}\left(t^{\prime}\right)$. It is clearly that the results obtained by the simplified GKS and the full GKS agree very well with each other.

We repeat the calculations with a larger turbulence Mach number $\mathrm{Ma}_{\mathrm{t}}=0.5$ and a larger CFL number $\mu_{\mathrm{CFL}}=0.5$. The results of the kinetic energy $K\left(t^{\prime}\right) / K_{0}$ and the dissipation rate $\varepsilon\left(t^{\prime}\right) / \varepsilon_{0}$ are shown in Fig. 13, and that of the skewness $S_{u}\left(t^{\prime}\right)$ and the flatness $F_{u}\left(t^{\prime}\right)$ are shown in Fig. 14. For the kinetic energy and the dissipation rate, the results computed from the simplified GKS and the full GKS are indistinguishable, as shown Fig. 13. As for the skewness $S_{u}\left(t^{\prime}\right)$ and the flatness $F_{u}\left(t^{\prime}\right)$, the results computed from the both schemes agree very well when $t^{\prime}<2.5$ but show observable differences later, especially for the flatness $F_{u}\left(t^{\prime}\right)$. Given the fact that the hydrodynamic variables and their derivatives are treated so differently at cell boundaries in the numerics in these two schemes, the agreement of the results is remarkable.

In the simplified GKS the hydrodynamic variables and their gradients are treated as continuous variables, this approach becomes numerically unstable when the turbulence Mach number is sufficiently high and shocks in the flow are so strong that they are practically discontinuous with the given resolution. It has been shown that there are eddy shocklets in the compressible DHIT [4]. To demonstrate this point, we show in Fig. 15 the contours of instantaneous den- 

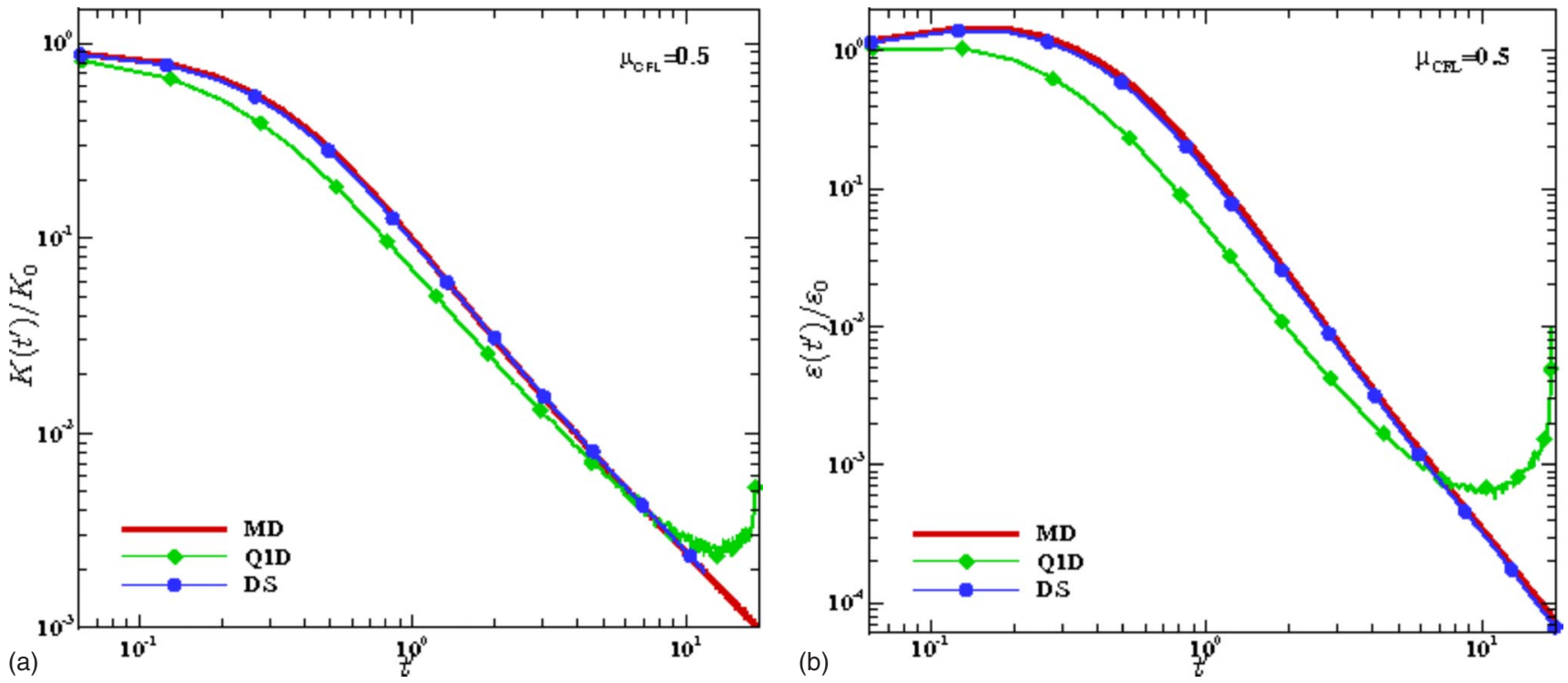

FIG. 9. (Color online) The evolution of the kinetic energy $K\left(t^{\prime}\right) / K_{0}$ (left) and dissipation rate $\varepsilon(t) / \varepsilon_{0}$ (right). Similar to Fig. 5, with $\mu_{\mathrm{CFL}}=0.5$.

sity $\rho$ and local Mach number Ma for a simulation of compressible DHIT with $\operatorname{Re}_{\lambda}=72.0$ and $\mathrm{Ma}_{\mathrm{t}}=0.5$ by using the simplified GKS scheme. The figure clearly shows areas where the gradients of $\rho$ and Ma have very high intensities, indicating the presence of shocklets.

To test the limit of the turbulence Mach number $\mathrm{Ma}_{\mathrm{t}}$ for the simplified GKS, we perform simulations with a fixed CFL number $\mu_{\mathrm{CFL}}=0.5$ and a fixed Reynolds number $\operatorname{Re}_{\lambda}$ $=72.0$ and various values of the initial turbulence Mach number $\mathrm{Ma}_{\mathrm{t}}$. Figure 16 shows the results for the kinetic energy $K\left(t^{\prime}\right) / K_{0}$ and the dissipation rate $\varepsilon(t) / \varepsilon_{0}$ obtained by using the simplified GKS with $\mathrm{Ma}_{\mathrm{t}}=0.1,0.5$, and 0.6. The corresponding results for the skewness $S_{u}\left(t^{\prime}\right)$ and the flatness $F_{u}\left(t^{\prime}\right)$ are shown in Fig. 17. Clearly, as the turbulence Mach number $\mathrm{Ma}_{\mathrm{t}}$ increases, so also do the strengths of shocklets

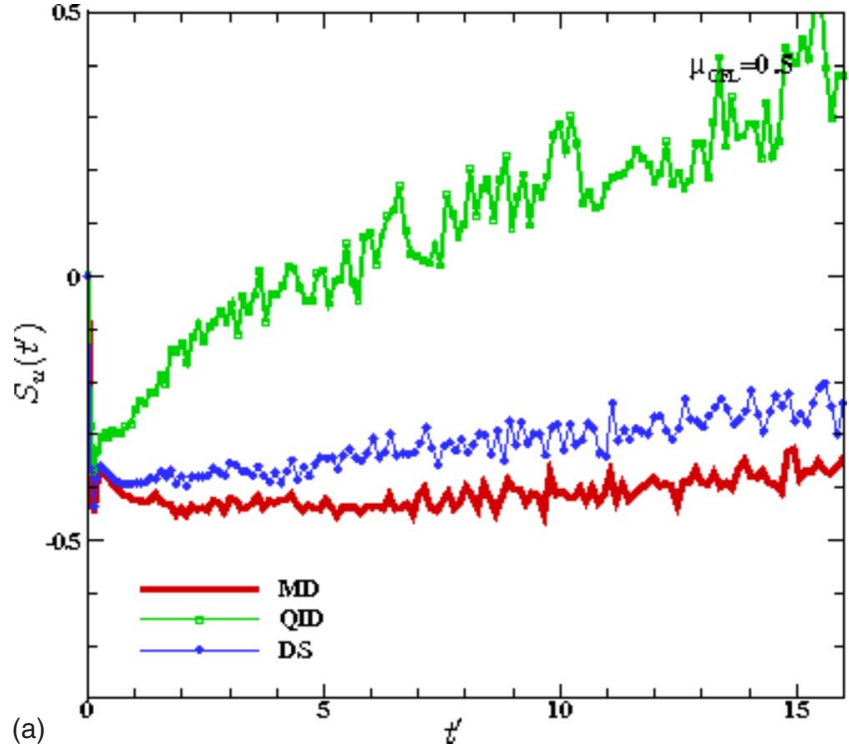

in the flow. Consequently, the gradients of flow fields become larger and larger as $\mathrm{Ma}_{\mathrm{t}}$ increases. This phenomenon is clearly reflected in both the skewness $S_{u}\left(t^{\prime}\right)$ and the flatness $F_{u}\left(t^{\prime}\right)$ shown in Fig. 17: as $\mathrm{Ma}_{\mathrm{t}}$ increases, the amplitudes of oscillations in both $S_{u}\left(t^{\prime}\right)$ and $F_{u}\left(t^{\prime}\right)$ increase significantly. The oscillation amplitudes also grow in time. If the initial turbulence Mach number $\mathrm{Ma}_{\mathrm{t}}$ is increased to 0.65 , the simulation becomes unstable quickly regardless of how small the CFL number $\mu_{\mathrm{CFL}}$ is. Our results indicate that the upper limit of the initial turbulence Mach number $\mathrm{Ma}_{\mathrm{t}}$, below which the simplified GKS can yield acceptable results, is about 0.6. Below this limit of $\mathrm{Ma}_{\mathrm{t}}$, the simplified GKS works effectively for the compressible DHIT.

We note that the simplified GKS is much more efficient computationally-it is about five times faster than the full

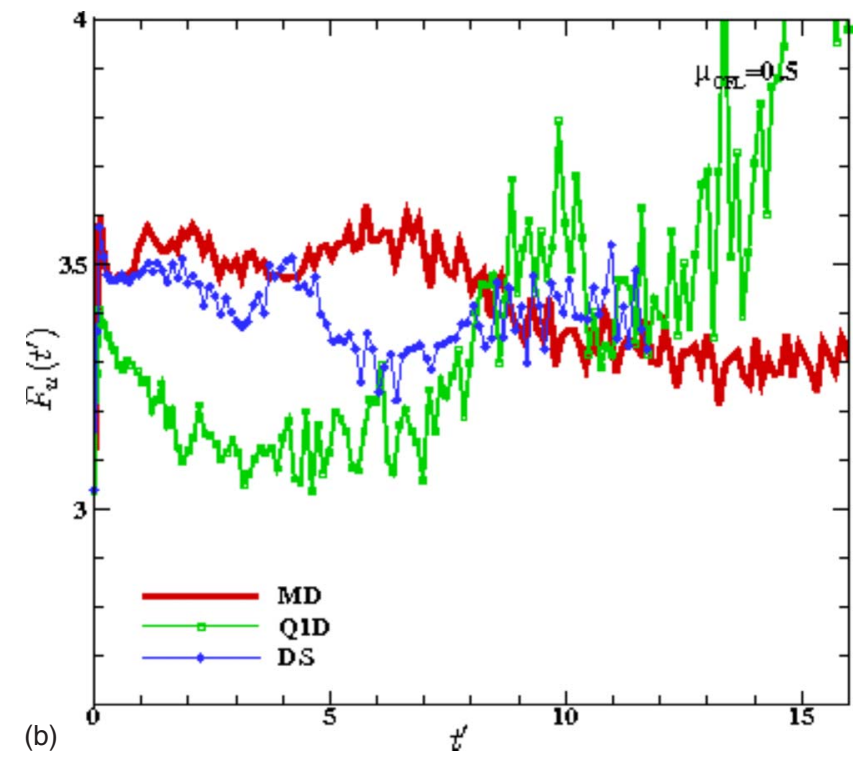

FIG. 10. (Color online) The evolution of the skewness $S_{u}\left(t^{\prime}\right)$ (left) and flatness $F_{u}\left(t^{\prime}\right)$ (right). Similar to Fig. 6 , with $\mu_{\mathrm{CFL}}=0.5$. 

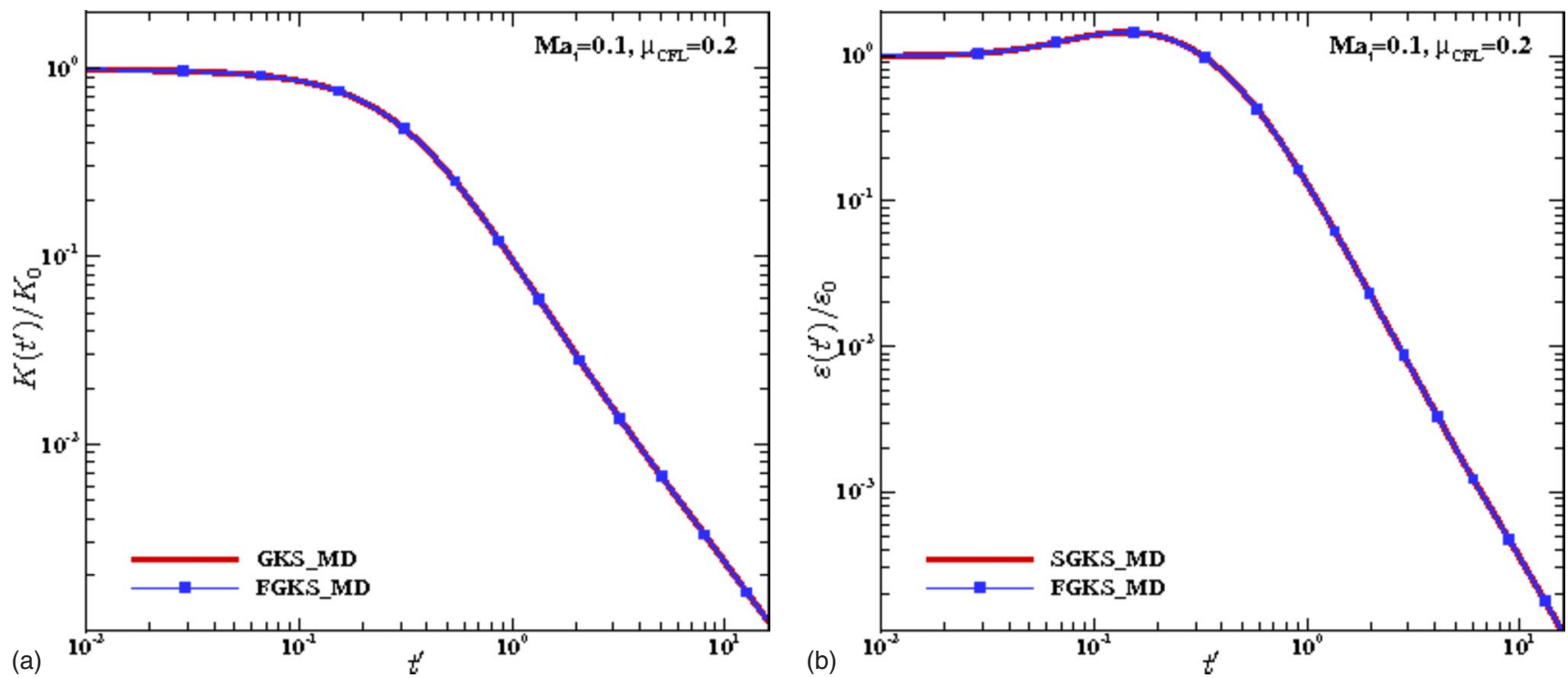

FIG. 11. (Color online) Evolutions of the kinetic energy $K\left(t^{\prime}\right) / K_{0}$ (left) and the dissipation rate $\varepsilon\left(t^{\prime}\right) / \varepsilon_{0}$ (right) obtained by the simplified GKS and the full GKS. $N^{3}=128^{3}, \operatorname{Re}_{\lambda}=72.0, \mathrm{Ma}_{\mathrm{t}}=0.1$, and $\mu_{\mathrm{CFL}}=0.2$.

multidimensional GKS. Thus it is highly recommended for flows for which the simplified GKS is suitable.

\section{Effects of flux limiter and artificial dissipation}

In the previous section we show that, for compressible flows with a high enough turbulent Mach number, the simplified GKS with the continuous treatment of hydrodynamic variables and their gradients is inadequate. When dealing with high Mach number turbulence, some sort of discontinuous treatment of shocks must be used. In addition to treating hydrodynamic variables and their gradients as discontinuities at cell boundaries, a flux limiter has to be used when the Mach number is sufficient high. Inevitably flux limiters do

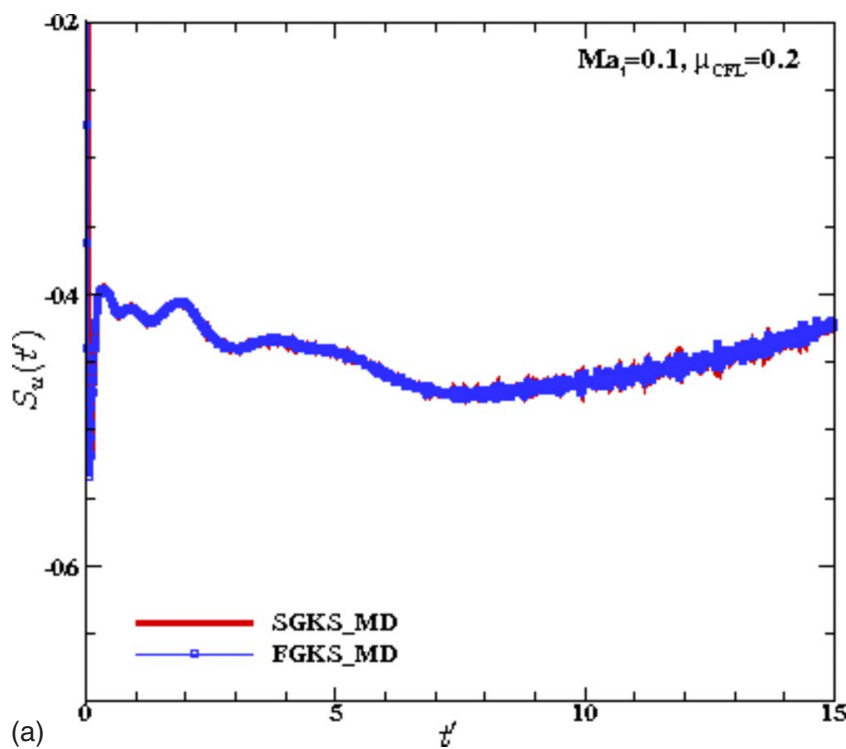

introduce numerical dissipations. In this section we will assess the effects of a flux limiter, as well as artificial dissipation on the DNS of compressible turbulence.

We use the full MD GKS with the van Leer limiter $[31,66]$ for the following tests. With the limiter, we can use the GKS to simulate compressible DHIT with a turbulence Mach number up to $\mathrm{Ma}_{\mathrm{t}}=2.0$. Figure 18 compares the results of the kinetic energy $K\left(t^{\prime}\right) / K_{0}$ and the dissipation rate $\varepsilon\left(t^{\prime}\right) / \varepsilon_{0}$ computed by using the full MD-GKS with and without the limiter. The flow conditions are $\operatorname{Re}_{\lambda}=72.0$ and $\mathrm{Ma}_{\mathrm{t}}$ $=0.5$, and the mesh size is $N^{3}=128^{3}$ and the CFL number is $\mu_{\mathrm{CFL}}=0.5$. This is the case for which the limiter is not necessary. The evolutions of the kinetic energy and the dissipation rate clearly show that the limiter introduces a significant

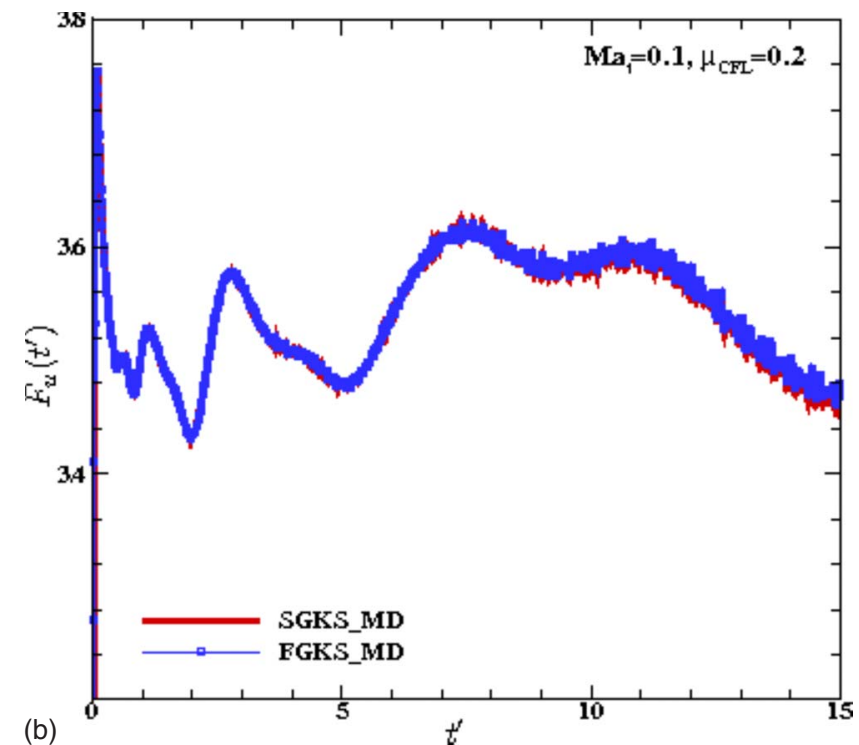

FIG. 12. (Color online) Evolutions of the skewness $S_{u}\left(t^{\prime}\right)$ (left) and the flatness $F_{u}\left(t^{\prime}\right)$ (right) obtained by the simplified GKS and the full GKS. $N^{3}=128^{3}, \operatorname{Re}_{\lambda}=72.0, \mathrm{Ma}_{\mathrm{t}}=0.1$, and $\mu_{\mathrm{CFL}}=0.2$. 

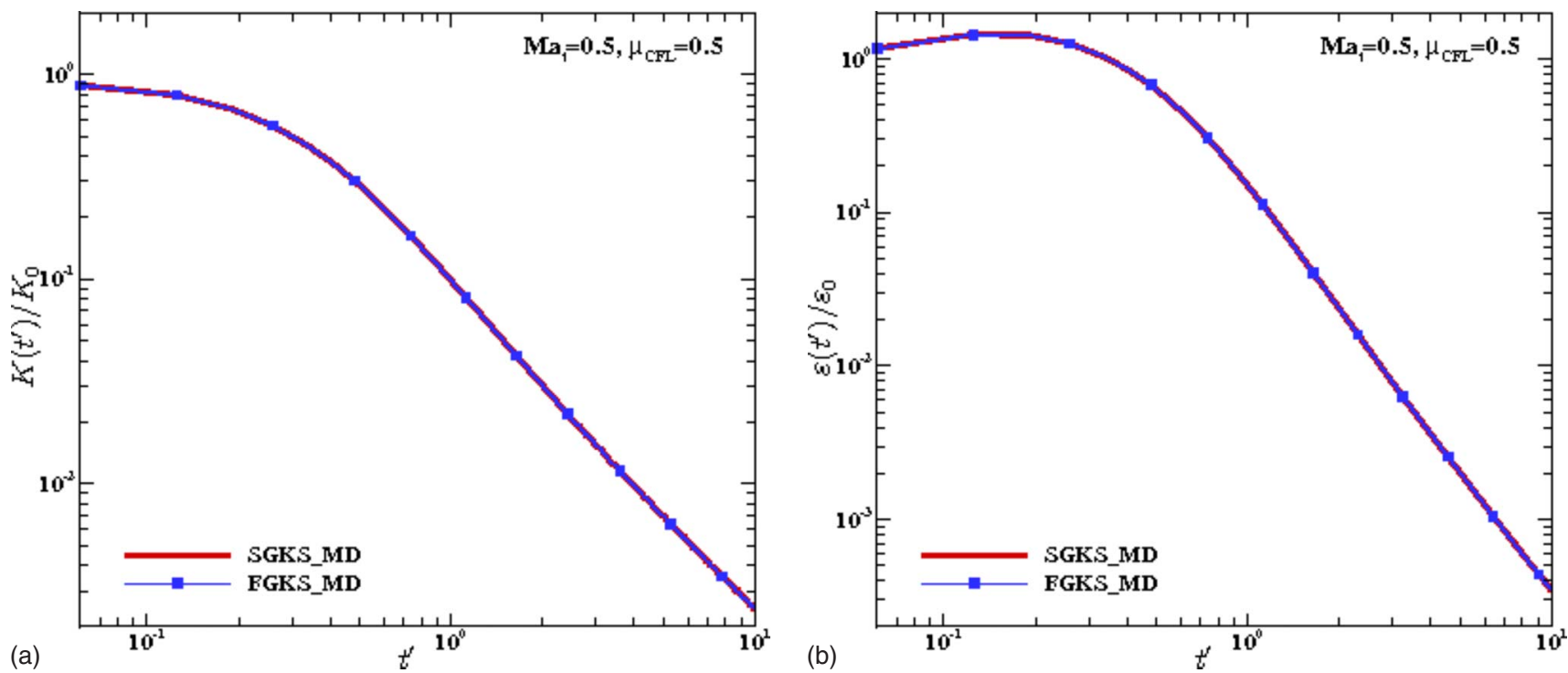

FIG. 13. (Color online) Evolutions of the kinetic energy $K\left(t^{\prime}\right) / K_{0}$ (left) and the dissipation rate $\varepsilon\left(t^{\prime}\right) / \varepsilon_{0}$ (right) obtained by the simplified GKS and the full GKS. Similar to Fig. 11, with $\mathrm{Ma}_{\mathrm{t}}=0.5$ and $\mu_{\mathrm{CFL}}=0.5$.

amount of numerical dissipation: the kinetic energy $K\left(t^{\prime}\right) / K_{0}$ and the dissipation rate computed with the limiter are much lower than their counterparts computed without the limiter. The results of the skewness $S_{u}\left(t^{\prime}\right)$ and the flatness $F_{u}\left(t^{\prime}\right)$ shown in Fig. 19 corroborate the above observation. Before the viscous decay completely dominates the decaying process [71], when $t^{\prime}<5.0$, the flatness $F_{u}\left(t^{\prime}\right)$ computed without the limiter is closer to its theoretical value of 3.5 than that with the limiter. Our results clearly demonstrate that flux limiters can introduce significant amount of numerical dissipation, which can adversely affect the quality of DNS results for compressible turbulence.

To assess the effect of the artificial dissipation, we use the van Leer limiter, plus the artificial dissipation with $\sigma=1.0$ in Eq. (24). Note that, without a limiter, the values of hydrody-

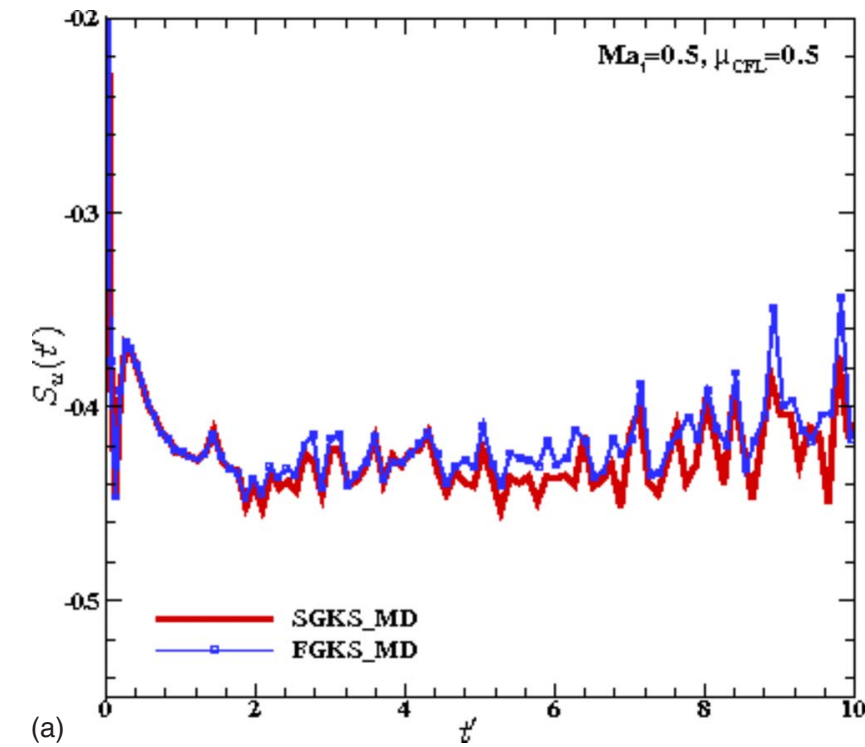

namic variables in both sides of a cell boundary are equal because of the linear interpolations used to compute these values [cf. Eqs. (16) and (18) and Fig. 1]. Therefore the artificial dissipation will not take effect unless a limiter is used in the GKS used in this work. In Figs. 18 and 19 we also show the effects of the artificial dissipation on $K\left(t^{\prime}\right) / K_{0}$, $\varepsilon\left(t^{\prime}\right) / \varepsilon_{0}, S_{u}\left(t^{\prime}\right)$, and $F_{u}\left(t^{\prime}\right)$ obtained by using the MD-GKS with the van Leer limiter and with $\sigma=1.0$ in Eq. (24). Clearly, the artificial dissipation has no visible effect on these quantities. This indicates that when a limiter is working, the dissipation due to the limiter is overwhelmingly dominant, and the effect of artificial dissipation is negligible for the low-Mach-number cases tested here.

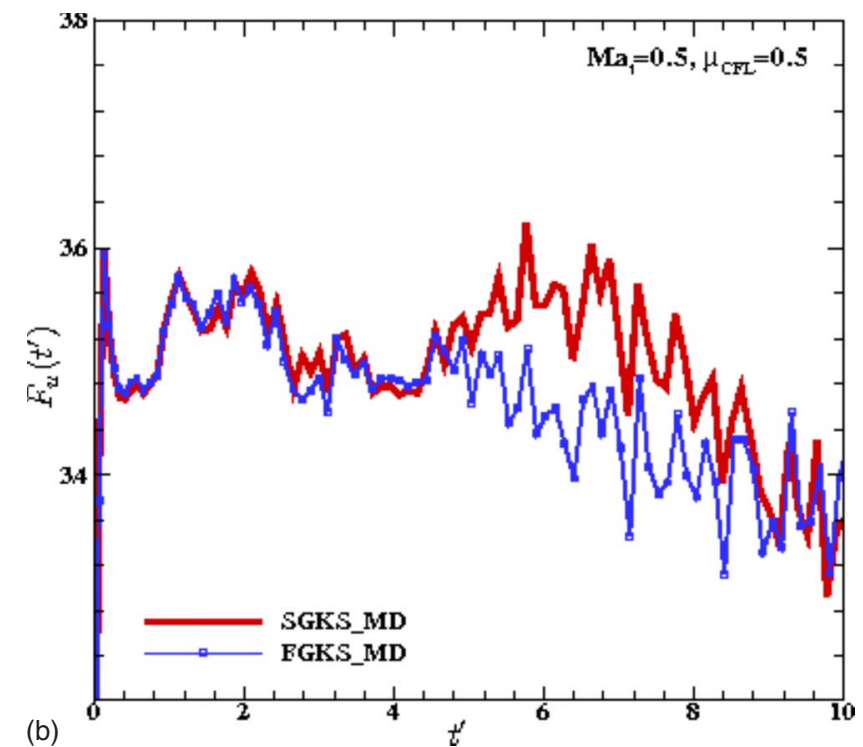

FIG. 14. (Color online) Evolutions of the skewness $S_{u}\left(t^{\prime}\right)$ (left) and the flatness $F_{u}\left(t^{\prime}\right)$ (right) obtained by the simplified GKS and the full GKS. Similar to Fig. $12, \mathrm{Ma}_{\mathrm{t}}=0.5$ and $\mu_{\mathrm{CFL}}=0.5$. 


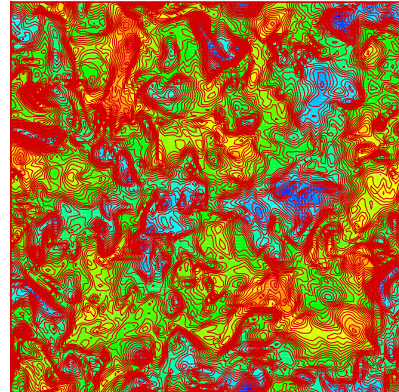

(a)

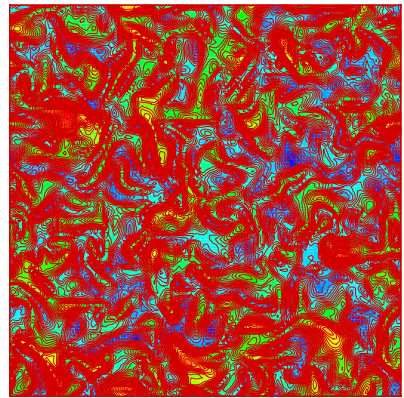

(b)
FIG. 15. (Color online) Contours of the density (left) and the Mach number (right) on the $x y$ plane $k=2$ at $t^{\prime}=1.03$ for $\mu_{\mathrm{CFL}}$ $=0.5, \mathrm{Ma}_{\mathrm{t}}=0.5, \mathrm{Re}_{\lambda}=72.0$, and $N^{3}=128^{3}$.

\section{Effect of the interpolation accuracy at cell boundaries}

In the GKS, one must interpolate hydrodynamic variables and their gradients from cell centers to cell boundaries. The accuracy of interpolations determines the accuracy of the scheme. Since the GKS is constructed as a second-order scheme, the linear interpolations of Eq. (34) are sufficient to achieve the required second-order accuracy. Because turbulence DNS have a very stringent requirement on the accuracy of numerical schemes, we would like to investigate the numerical fidelity of the linear interpolations used in the GKS for DNS of compressible DHIT. In what follow, we will use the simplified GKS to test the linear interpolation of Eq. (34) against the third-order interpolations of Eq. (35). We maintain the Reynolds number $\operatorname{Re}_{\lambda}=72.0$ and the mesh size $N^{3}$ $=128^{3}$.

In Fig. 20, we show the kinetic energy and the dissipations rate with two sets of conditions: $\mathrm{Ma}_{\mathrm{t}}=0.1$ and $\mu_{\mathrm{CFL}}$ $=0.2$ and $\mathrm{Ma}_{\mathrm{t}}=0.5$ and $\mu_{\mathrm{CFL}}=0.4$. For the case of $\mathrm{Ma}_{\mathrm{t}}=0.1$, the kinetic energy $K\left(t^{\prime}\right) / K_{0}$ shows no visible difference due to the difference of interpolations used, while the dissipation rate clear indicates that the linear interpolations are more dissipative than the third-order ones-the peak of $\varepsilon\left(t^{\prime}\right) / \varepsilon$ computed with the linear interpolations is lower than what computed with the third-order interpolations. For the case of $\mathrm{Ma}_{\mathrm{t}}=0.5$, the discrepancy in the $\varepsilon\left(t^{\prime}\right) / \varepsilon$ due to interpolations disappears. However, the kinetic energy $K\left(t^{\prime}\right) / K_{0}$ computed with the linear interpolations decays slower after $t^{\prime}>8.0$, indicating that a larger numerical dissipation due to linear interpolations. The reason behind this phenomenon can be explained as follows. Stronger numerical dissipations due to linear interpolations reduce the dissipation rate $\varepsilon$ in the initial stage, as clearly shown in Fig. 20 for the case of $\mathrm{Ma}_{\mathrm{t}}=0.1$, which in turn slow down the decay of the kinetic energy $K$ in later times. Thus, a larger numerical dissipation leads to a slower decay of the kinetic energy in this case.

Figures 21 and 22 show the evolution of $S_{u}\left(t^{\prime}\right)$ and $F_{u}\left(t^{\prime}\right)$ for $\mathrm{Ma}_{\mathrm{t}}=0.1$ and $\mathrm{Ma}_{\mathrm{t}}=0.5$, respectively, corresponding to the results shown in Fig. 20. For the case of $\mathrm{Ma}_{\mathrm{t}}=0.1$, the results in Fig. 21 show that the results computed with linear and third-order interpolations display large discrepancies. While one cannot argue that the results computed with the thirdorder interpolations are better than that computed with the linear interpolations, there are some indications-the flatness $S_{u}\left(t^{\prime}\right)$ computed with the third-order interpolations is bounded between -0.5 and -0.4 , which is more reasonable than that computed with the linear interpolations, which goes beyond the bound of $(-0.5,-0.4)$ after $t^{\prime}>15.0$. For the case of $\mathrm{Ma}_{\mathrm{t}}=0.5$, oscillations in the $S_{u}\left(t^{\prime}\right)$ and $F_{u}\left(t^{\prime}\right)$ computed with the linear interpolations have larger amplitudes. However, the smoothed $S_{u}\left(t^{\prime}\right)$ and $F_{u}\left(t^{\prime}\right)$ computed with the linear and third-order interpolations agree with each other rather well, as shown in Fig. 22.

Our results here clear show that the accuracy of interpolations does have observable effects on various quantities in DNS of compressible DHIT. Clearly, the linear interpolations introduce stronger numerical dissipations. These effects seem to be stronger at lower Mach numbers. Overall, the linear
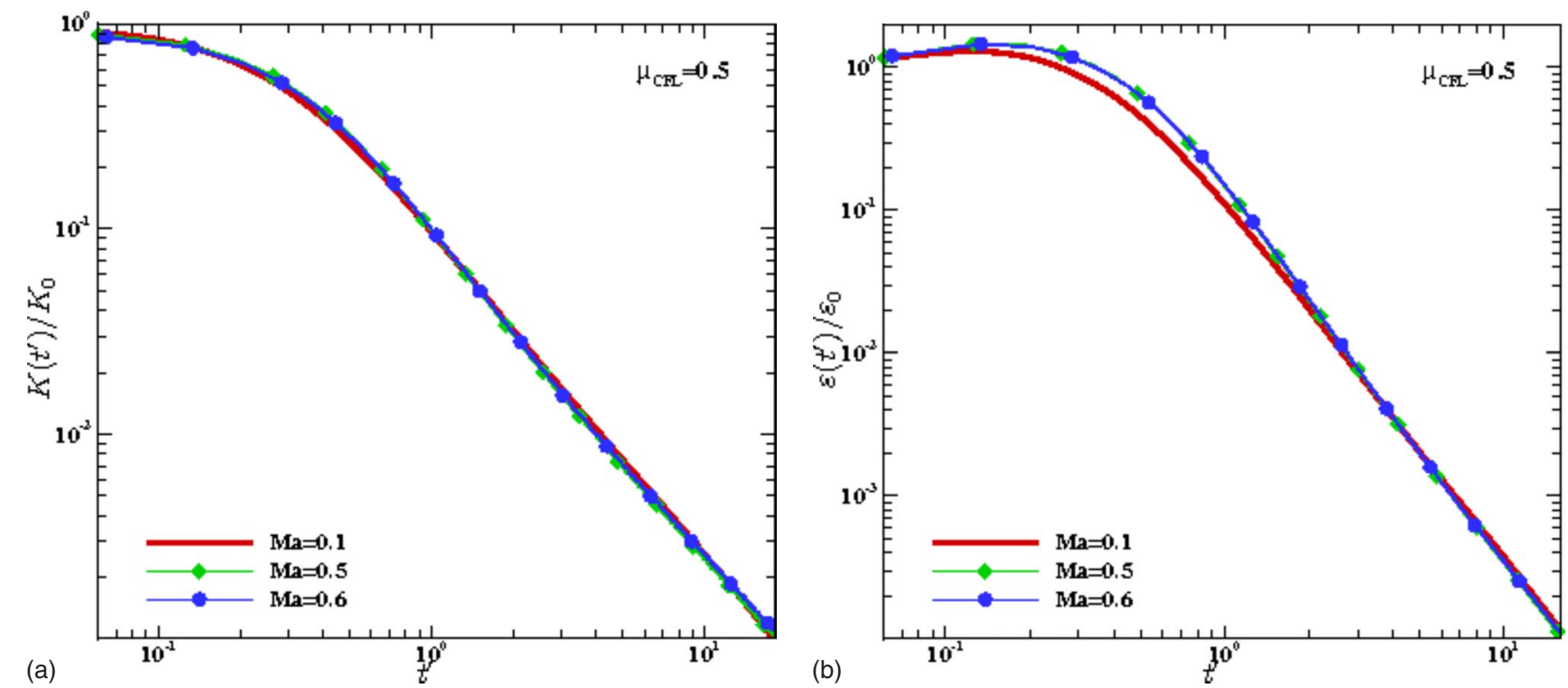

FIG. 16. (Color online) The evolution of $K\left(t^{\prime}\right) / K_{0}$ (left) and $\varepsilon\left(t^{\prime}\right) / \varepsilon_{0}$ (right) obtained by using the simplified GKS with $N^{3}=128^{3}$, $\mu_{\mathrm{CFL}}=0.5, \mathrm{Re}_{\lambda}=72.0$, and $\mathrm{Ma}_{\mathrm{t}}=0.1,0.5$, and 0.6 . 

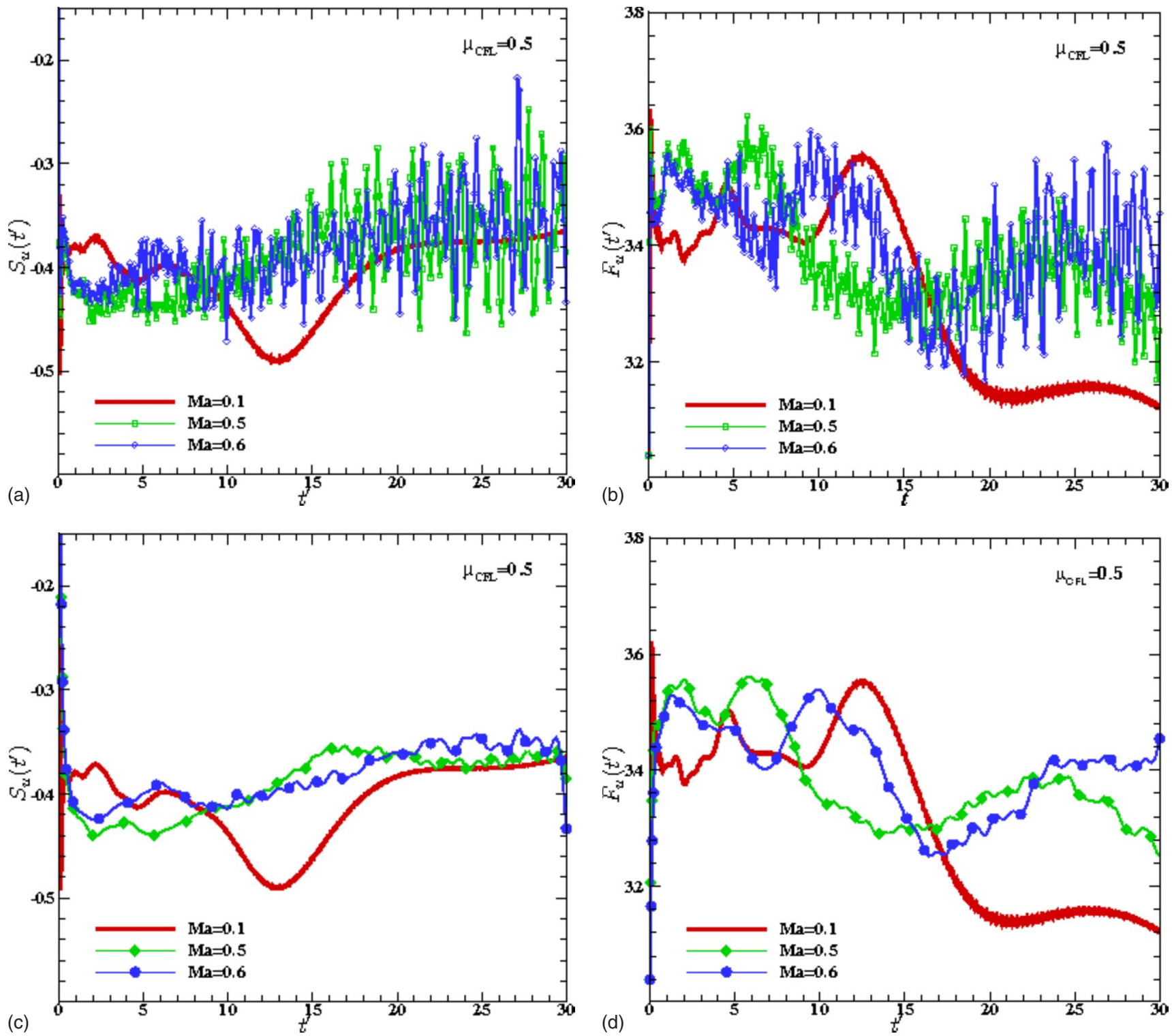

FIG. 17. (Color online) The evolution of $S_{u}(t)$ (left) and $F_{u}(t)$ (right) obtained by using the simplified GKS with $N^{3}=128^{3}, \mu_{\mathrm{CFL}}=0.5$, $\operatorname{Re}_{\lambda}=72.0$, and $\mathrm{Ma}_{\mathrm{t}}=0.1,0.5$, and 0.6. In the bottom row, the results are smoothed.

interpolations are acceptable for DNS of compressible turbulence flows. Overall, the linear interpolations save about $15 \%$ computational time when compared with the third-order interpolations.

\section{E. Effect of bulk viscosity}

Finally, we assess the effect of the bulk viscosity $\eta$. We use the simplified GKS with linear interpolations at cell boundaries. In GKS, the bulk viscosity is tuned with the parameter $Z$ and it does not change the complexity of the code because one does not need to explicitly compute the divergence of the velocity field $\boldsymbol{\nabla} \cdot \boldsymbol{u}$. We perform the simulations for the compressible DHIT at $\mathrm{Ma}_{\mathrm{t}}=0.1$ and 0.5 with $(K=2)$ or without $(K=0)$ the bulk viscosity $\eta$. Figure 23 shows the effect of the bulk viscosity at $\mathrm{Ma}_{\mathrm{t}}=0.1$ on the kinetic energy $K\left(t^{\prime}\right) / K_{0}$ and the dissipation rate $\varepsilon\left(t^{\prime}\right) / \varepsilon_{0}$. We can see that the bulk viscosity has no observable effect on the kinetic energy $K\left(t^{\prime}\right) / K_{0}$; however, it does increase the dissipation rate $\varepsilon\left(t^{\prime}\right) / \varepsilon_{0}$ slightly in the initial stage. Clearly, the dissipation due to the bulk viscosity $\eta$ produces observable effects in both the skewness $S_{u}\left(t^{\prime}\right)$ and the flatness $F_{u}\left(t^{\prime}\right)$, as shown in Fig. 24.

When the initial turbulent Mach number $\mathrm{Ma}_{\mathrm{t}}=0.5$, the bulk viscosity $\eta$ does not seem to have any observable effects on both the kinetic energy $K\left(t^{\prime}\right) / K_{0}$ and the dissipation rate $\varepsilon\left(t^{\prime}\right) / \varepsilon_{0}$, as shown in Fig. 25 . However, the bulk viscosity do have prominent effects on both the skewness $S_{u}\left(t^{\prime}\right)$ and the flatness $F_{u}\left(t^{\prime}\right)$, as shown in Fig. 26. Several observations can be made here. First, for a low initial turbulence Mach number $\mathrm{Ma}_{\mathrm{t}}=0.1$, the bulk viscosity $\eta$ simply adds more dissipation to the flow; it enhances the value of the skewness $S_{u}\left(t^{\prime}\right)$ and depresses that of the flatness $F_{u}\left(t^{\prime}\right)$ after an initial period of time. It seems to be difficult to distinguish a priori between the dissipative effects due to the bulk vis- 

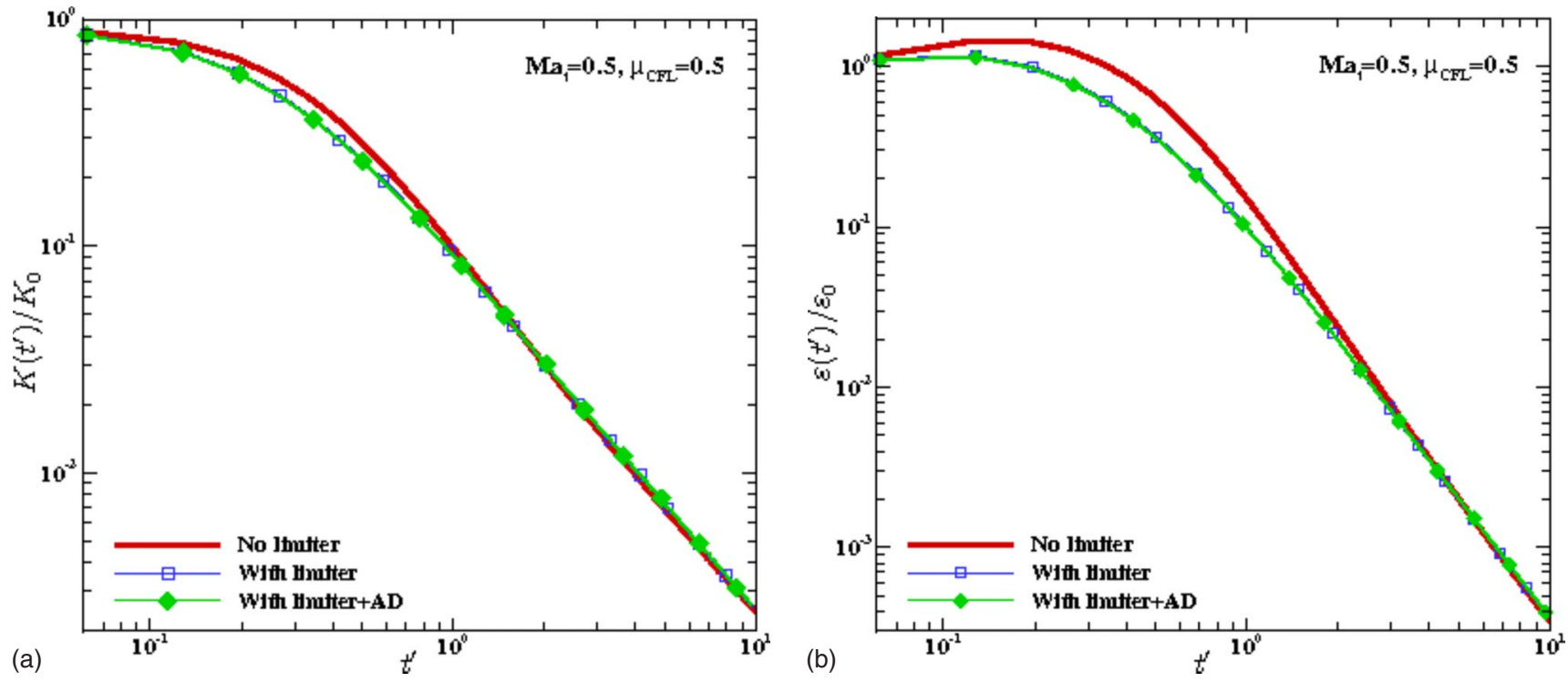

FIG. 18. (Color online) Effects of flux limiter and artificial dissipation (AD) on the kinetic energy $K\left(t^{\prime}\right) / K_{0}($ left $)$ and $\varepsilon\left(t^{\prime}\right) / \varepsilon_{0}$ (right). We set $\sigma=1.0$ in Eq. (24) when $\mathrm{AD}$ is used. $\operatorname{Re}_{\lambda}=72.0, \mathrm{Ma}_{\mathrm{t}}=0.5, \mu_{\mathrm{CFL}}=0.5$, and $N^{3}=128^{3}$.

cosity $\eta$ and that due to numerical viscosities. Secondly, for a higher initial turbulence Mach number $\mathrm{Ma}_{\mathrm{t}}=0.5$, the skewness $S_{u}\left(t^{\prime}\right)$ and the flatness $F_{u}\left(t^{\prime}\right)$ computed with a nonzero bulk viscosity have high-frequency oscillations stronger than that with zero bulk viscosity. In addition, it is interesting to note that the skewness $S_{u}\left(t^{\prime}\right)$ and the flatness $F_{u}\left(t^{\prime}\right)$ computed with a nonzero bulk viscosity remain much closer to their theoretical values of -0.5 and 3.5 at late stage of DHIT, respectively, than their counterparts with a zero bulk viscosity.

\section{CONCLUSIONS}

In this paper we apply the gas-kinetic scheme (GKS) for DNSs for compressible decaying homogeneous isotropic tur-

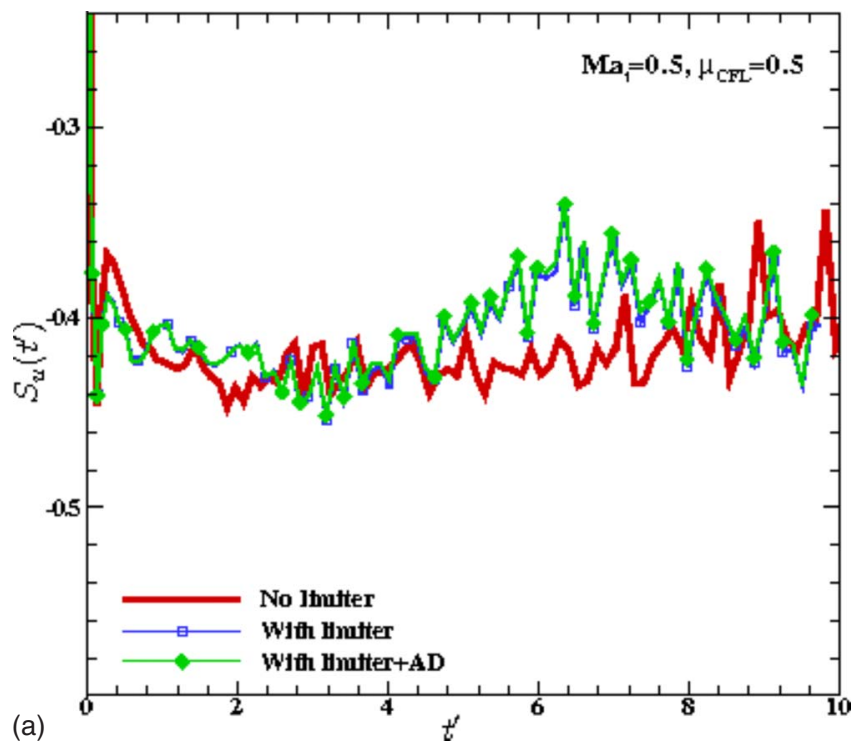

bulence in a three-dimensional cube with periodic boundary conditions. We measure the statistical quantities including the total kinetic energy $K\left(t^{\prime}\right)$, the dissipation rate $\varepsilon\left(t^{\prime}\right)$, the skewness $S_{u}\left(t^{\prime}\right)$, and the flatness $F_{u}\left(t^{\prime}\right)$. The simulations are carried out with the Taylor microscale Reynolds number $\operatorname{Re}_{\lambda}=72.0$, a fixed mesh size of $N^{3}=128^{3}$, and various values of initial turbulence Mach number $\mathrm{Ma}_{\mathrm{t}}$, up to the dimensionless time $t^{\prime} \approx 30$ in terms of the turbulence turnover time $\tau_{0}=K_{0} / \varepsilon_{0}$

We first validate our GKS code against pseudospectral simulations in both near incompressible and fully compressible regions for the DHIT, corresponding to the initial turbulence $\mathrm{Ma}_{\mathrm{t}}=0.1$ and 0.5 , respectively. We find that the GKS can yield satisfactory results for $K\left(t^{\prime}\right), \varepsilon\left(t^{\prime}\right), S_{u}\left(t^{\prime}\right)$, and

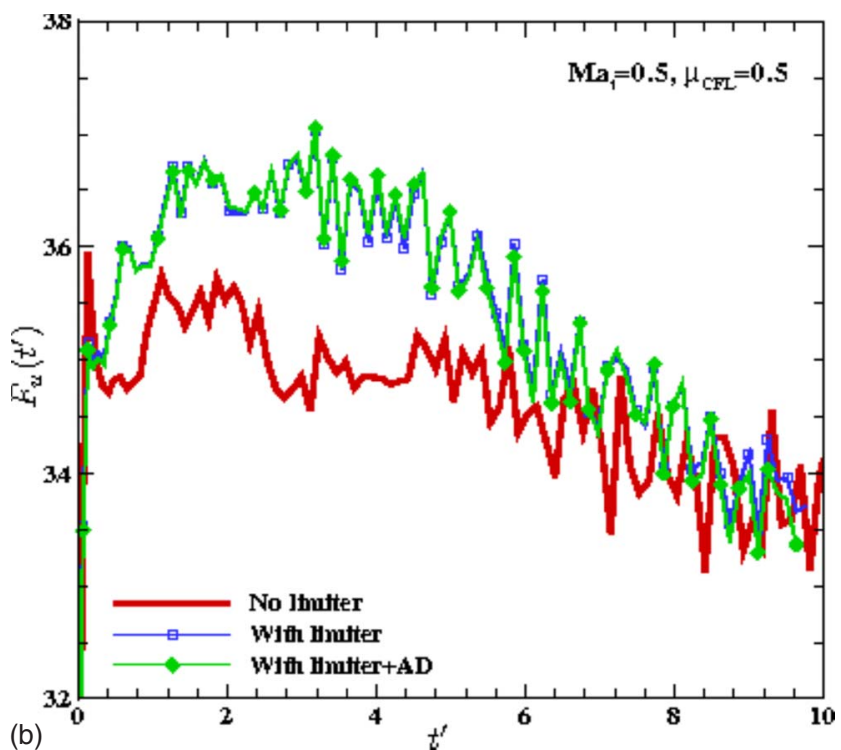

FIG. 19. (Color online) Effect of flux limiter and AD on the skewness $S_{u}(t)$ (left) and the flatness $F_{u}(t)$ (right). We set $\sigma=1.0 \mathrm{in}$ Eq. (24) when $\mathrm{AD}$ is used. $\operatorname{Re}_{\lambda}=72.0, \mathrm{Ma}_{\mathrm{t}}=0.5, \mu_{\mathrm{CFL}}=0.5$, and $N^{3}=128^{3}$. 

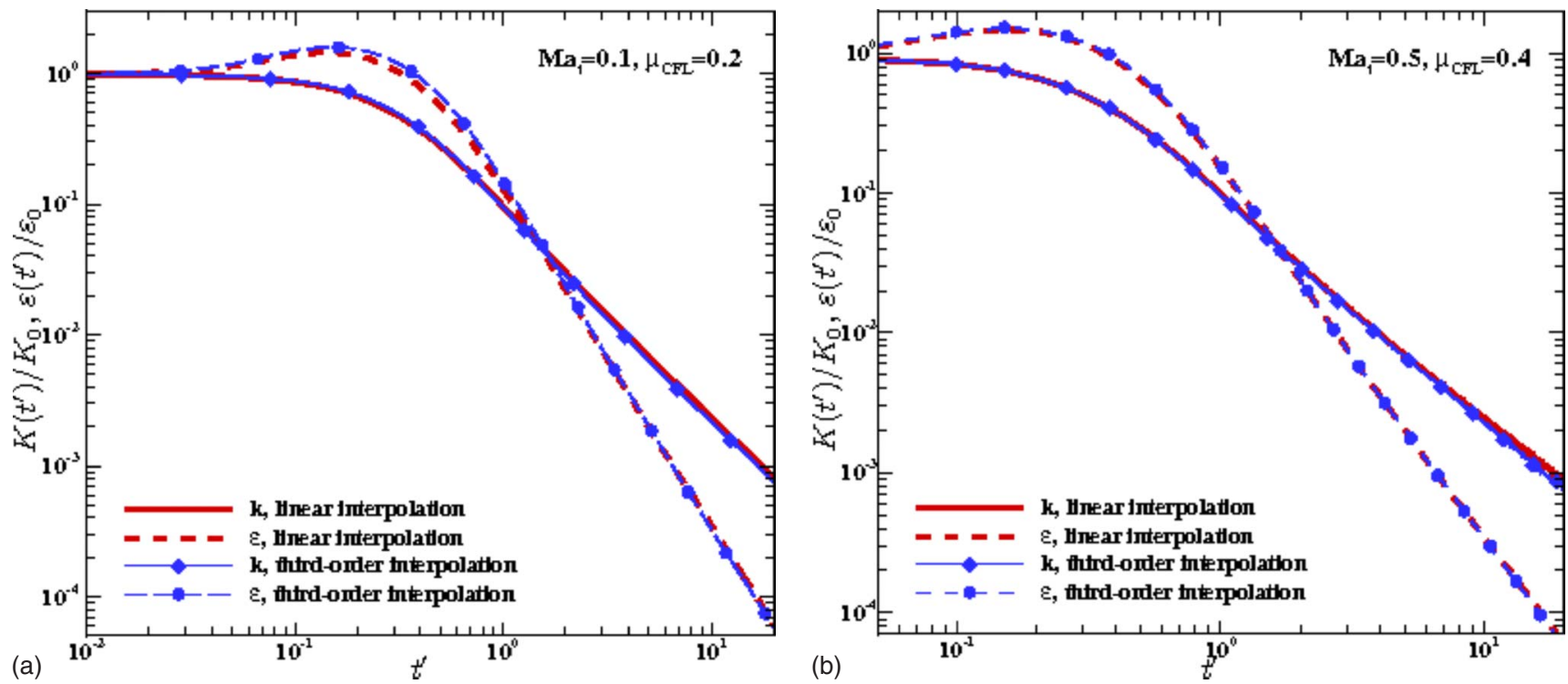

FIG. 20. (Color online) Effect of the accuracy of interpolations on the kinetic energy $K\left(t^{\prime}\right) / K_{0}$ and the dissipation rate $\varepsilon\left(t^{\prime}\right) / \varepsilon_{0}$. $N^{3}=128^{3}, \operatorname{Re}_{\lambda}=72.0, \mathrm{Ma}_{\mathrm{t}}=0.1$, and $\mu_{\mathrm{CFL}}=0.2$ (left); and $\mathrm{Ma}_{\mathrm{t}}=0.5$ and $\mu_{\mathrm{CFL}}=0.4$ (right).

$F_{u}\left(t^{\prime}\right)$, and the results are in good agreement with pseudospectral results.

We investigate effects due to approximations made in computing the fluxes, that is, we compare the quasi-onedimensional (Q1D) GKS and dimensional-splitting (DS) GKS, versus the full MD GKS. We find that the Q1D-GKS is the most dissipative and the least stable and accurate scheme among the three, while the full GKS is the best. The accuracy and numerical stability of the Q1D-GKS deteriorate as the initial turbulence Mach number $\mathrm{Ma}_{\mathrm{t}}$ increases, while the DS-GKS is only slightly more dissipative than the full MD GKS, which is only observable in the skewness and the flatness. For most part, the DS-GKS results agree well with the full MD-GKS ones in the parameter ranges we have tested.

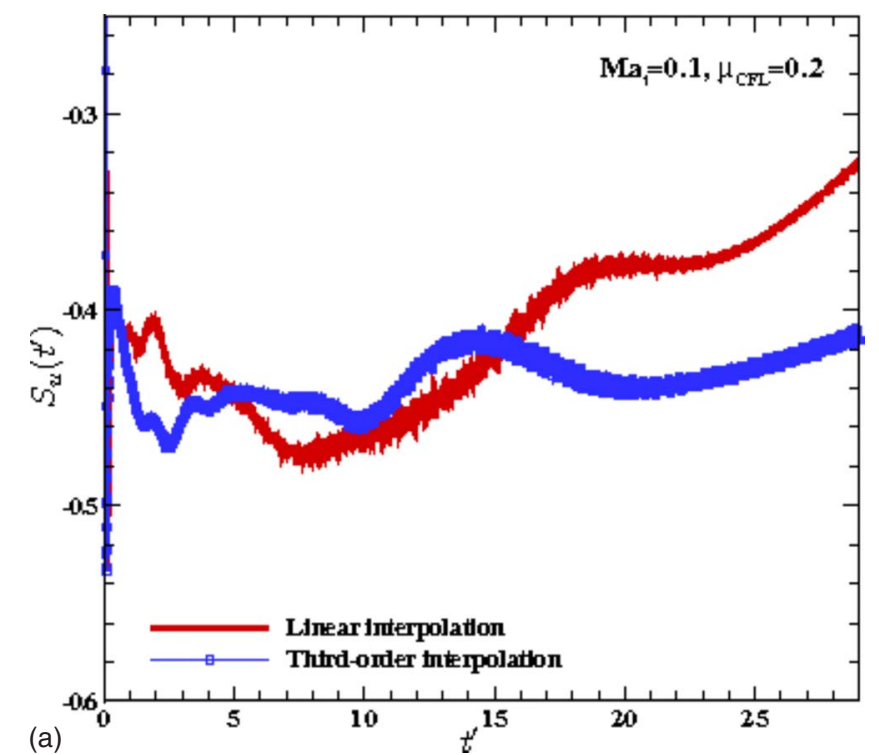

The ratio of the computational speeds of the DS-GKS and the full GKS is about 1.8. The tests performed in this work show that the DS-GKS is an adequate DNS tool to compute the low-order turbulence statistical quantities in compressible decaying turbulence, while the Q1D-GKS is not recommended for the purpose of turbulence DNS.

We test the simplified GKS for smooth flows. The simplified GKS treats the hydrodynamic variables and their derivatives as continuous variables at cell boundaries, leading to considerably simplifications in the calculations of fluxes, thus significantly reducing the computational cost. The computational cost of the simplified GKS is only about $1 / 5$ of that of the full GKS. The simplified GKS works very well for the DNS of decaying turbulence when the initial turbulence

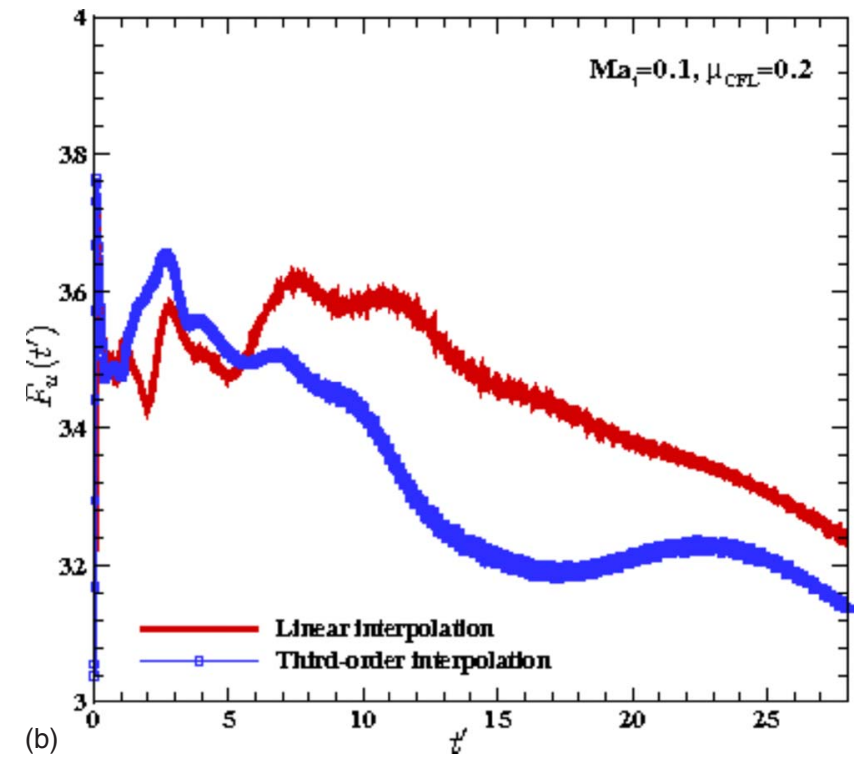

FIG. 21. (Color online) Effect of interpolation accuracy at cell boundaries on $S_{u}(t)$ (left) and $F_{u}(t)$ (right) at $\mu_{\mathrm{CFL}}=0.2, \mathrm{Ma}_{\mathrm{t}}=0.1$, $\operatorname{Re}_{\lambda}=72.0$, and $N^{3}=128^{3}$. 

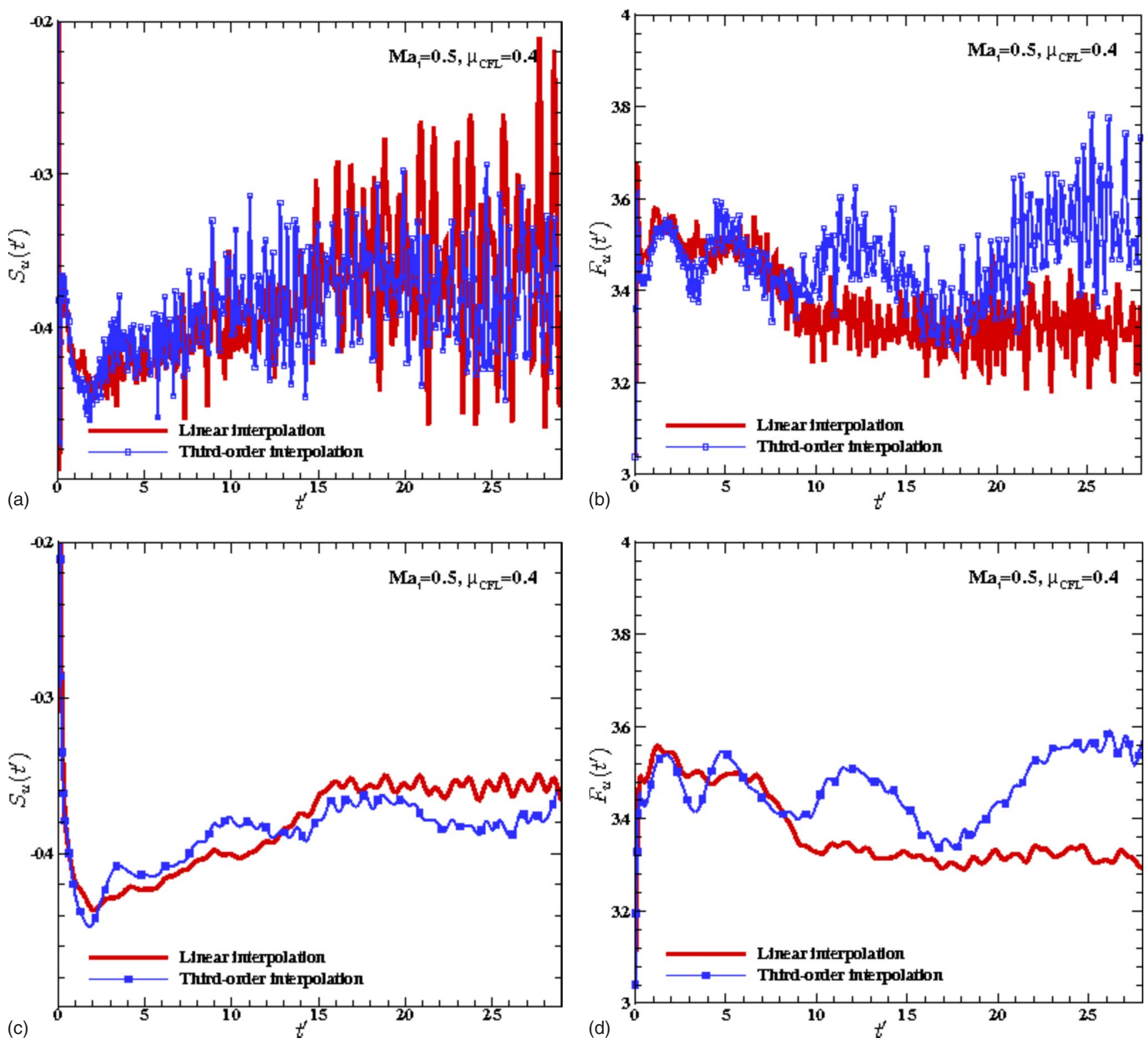

FIG. 22. (Color online) Effect of interpolation accuracy at cell boundaries on $S_{u}(t)$ (left) and $F_{u}(t)($ right $)$ at $\mu_{\mathrm{CFL}}=0.4, \mathrm{Ma}_{\mathrm{t}}=0.5$, $\operatorname{Re}_{\lambda}=72.0$ and $N^{3}=128^{3}$. In the bottom row, the results are smoothed.

Mach number $\mathrm{Ma}_{\mathrm{t}}<0.6$. At a low Mach number $\mathrm{Ma}_{\mathrm{t}}=0.1$, the results generated from both simplified and full GKS have almost invisible difference. At a higher Mach number $\mathrm{Ma}_{\mathrm{t}}$ $=0.5$, the differences can only be observed in the skewness $S_{u}\left(t^{\prime}\right)$ and the flatness $F_{u}\left(t^{\prime}\right)$ when $t^{\prime}:=t / \tau_{0}>5.0$, where $\tau_{0}$ $:=K_{0} / \varepsilon_{0}$ is the turbulence turnover time. The simplified GKS is highly recommended as a DNS tool for turbulence when the Mach number is not too high.

We also evaluate the effects on the low-order statistical turbulence quantities due to flux limiter, the accuracy of the interpolations at cell boundaries, and the bulk viscosity $\eta$. The flux limiter introduces considerable amount of numerical dissipations, thus significantly affects all the turbulence statistics adversely. Therefore it should only be used for high Mach number flows when necessary, e.g., when the initial turbulence Mach number $\mathrm{Ma}_{\mathrm{t}}>0.6$ for DHIT. We observe that the accuracy of the interpolations at cell boundaries has more significant effects near incompressible flow with low Mach number, e.g., $\mathrm{Ma}_{\mathrm{t}}=0.1$. With $\mathrm{Ma}_{\mathrm{t}}=0.5$, the flatness $F_{u}\left(t^{\prime}\right)$ is the only quantity showing visible difference due to the accuracy of the interpolations. Thus, higher-order interpolations would only be needed for near incompressible flows. The bulk viscosity $\eta$ introduces dissipations due to dilatation, which is a physical effect. The bulk viscosity has very little effect on the kinetic energy $K\left(t^{\prime}\right) / K_{0}$ and the dissipation rate $\varepsilon\left(t^{\prime}\right) / \varepsilon_{0}$, and its effect on $\varepsilon\left(t^{\prime}\right) / \varepsilon_{0}$ is more visible for near incompressible flows, i.e., $\mathrm{Ma}_{\mathrm{t}}=0.1$. For near incompressible flows, it appears that the bulk viscosity does nothing more than increasing viscous effect. At a higher Mach number $\mathrm{Ma}_{\mathrm{t}}=0.5$, the bulk viscosity seems to play a more subtle role. It enhances the intensities of highfrequency oscillations in the skewness $S_{u}\left(t^{\prime}\right)$ and the flatness $F_{u}\left(t^{\prime}\right)$, which are due to acoustics in the system. Also, the bulk viscosity seems to maintain the values of $S_{u}\left(t^{\prime}\right)$ and 

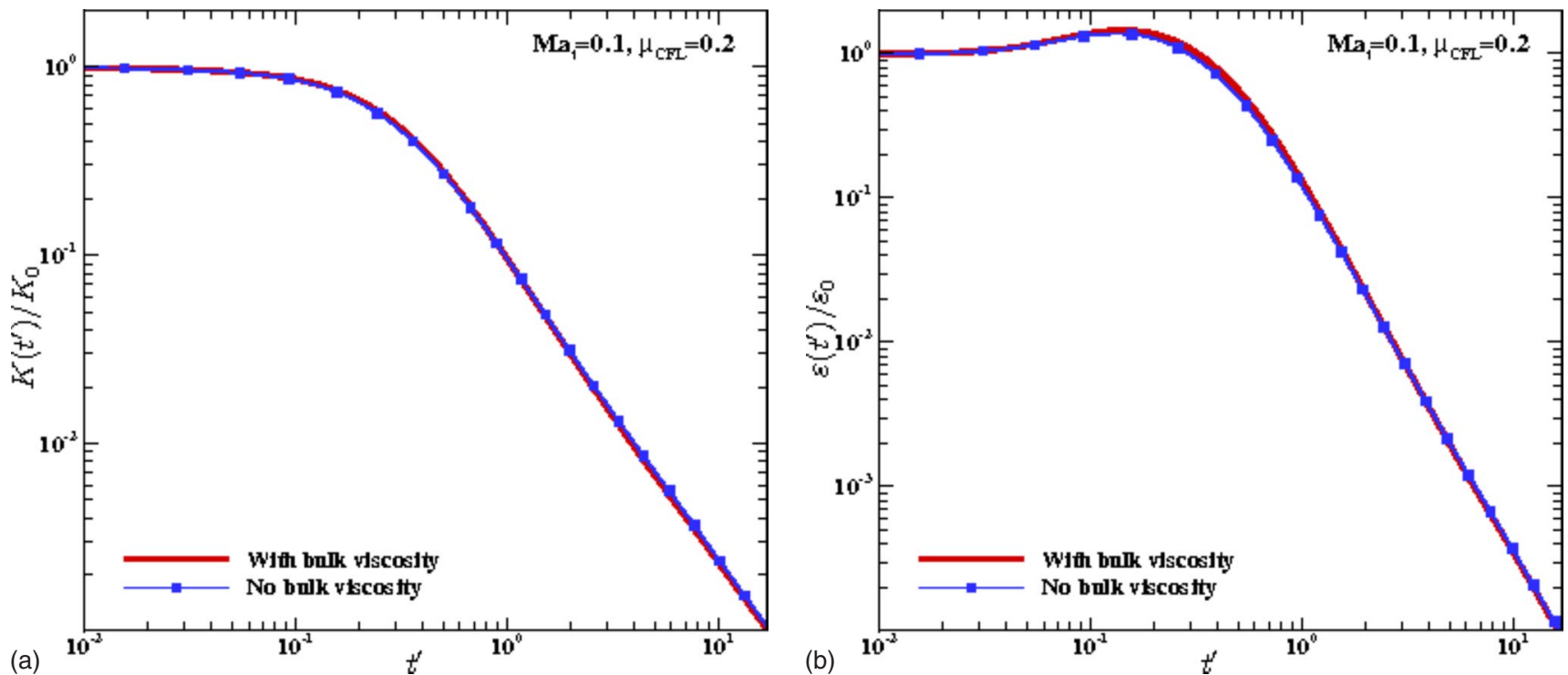

FIG. 23. (Color online) Effect of the bulk viscosity $\eta$ on the kinetic energy $K\left(t^{\prime}\right) / K_{0}$ and the dissipation rate $\varepsilon\left(t^{\prime}\right) / \varepsilon_{0}$. $N^{3}=128^{3}$, $\operatorname{Re}_{\lambda}=72.0, \mathrm{Ma}_{\mathrm{t}}=0.1$, and $\mu_{\mathrm{CFL}}=0.2$.

$F_{u}\left(t^{\prime}\right)$ closer to their theoretical ones, -0.5 and 3.5 , respectively, than the simulations without the bulk viscosity.

Overall, our results demonstrate that the gas-kinetic scheme is adequate to simulate decaying homogeneous isotropic turbulence as far as the low-order statistics are concerned. The GKS is not the most effective and efficient method for near incompressible flows with low Mach numbers [65]. For near incompressible flows, our experience indicates that the lattice Boltzmann equation (LBE) is a much better method in terms of effectiveness and efficiency $[61,72]$. Since the GKS is only a second-order scheme, the strength of the GKS may be in high-Mach-number flows in which a flux limiter must be used. When a flux limiter must be used, the benefit of using more accurate high-order methods may not be so obvious because the numerical dissipation

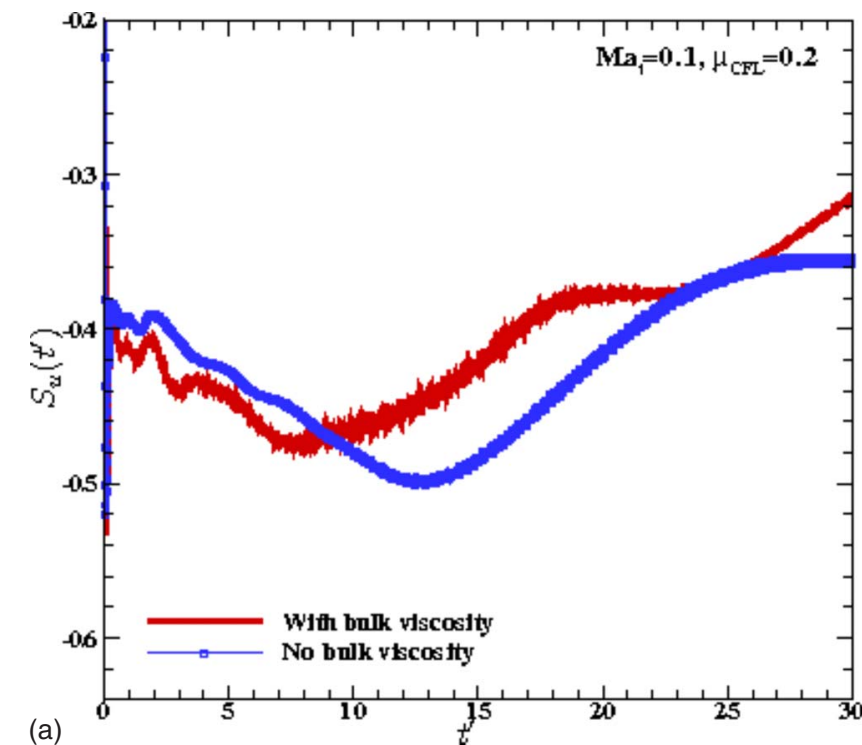

introduced by the flux limiter becomes the dominating factor affecting the accuracy of the scheme.

Kinetic schemes have two notable advantages in general, which motivate this work in part. The first one is the numerics. In contrast to the Navier-Stokes equation with a nonlinear advection term $\boldsymbol{u} \cdot \boldsymbol{\nabla} \boldsymbol{u}$, the Boltzmann equation has a linear advection term $\boldsymbol{\xi} \cdot \boldsymbol{\nabla} f=\boldsymbol{\nabla} \cdot(\boldsymbol{\xi} f)$ and its nonlinearity resides in the collision term which is local. This feature of the kinetic equation has important ramifications including [32]: (a) its nonlinearity is in local collision term, stiffness of which can be overcome by local techniques; and (b) it is much easier to formulate multidimensional schemes for fluxes. The second advantage of the GKS is physics. Kinetic schemes based on the Boltzmann equation have the potential to model extended hydrodynamics which is beyond the validity of the

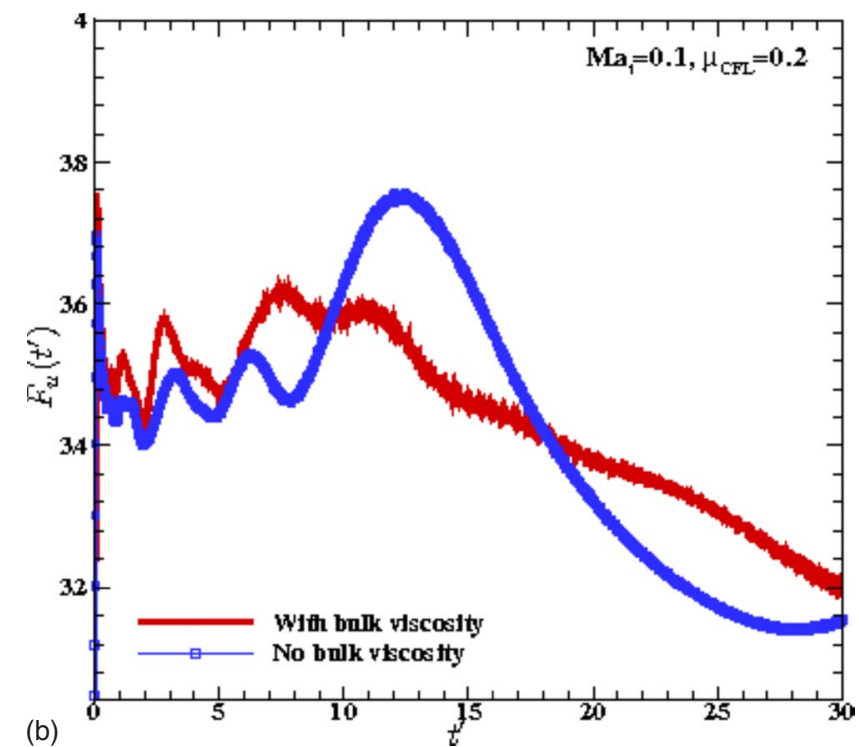

FIG. 24. (Color online) Effect of the bulk viscosity on the skewness $S_{u}(t)$ (left) and the flatness $F_{u}(t)(\operatorname{right}) \cdot N^{3}=128^{3}, \operatorname{Re}_{\lambda}=72.0$, $\mathrm{Ma}_{\mathrm{t}}=0.1$, and $\mu_{\mathrm{CFL}}=0.2$. 

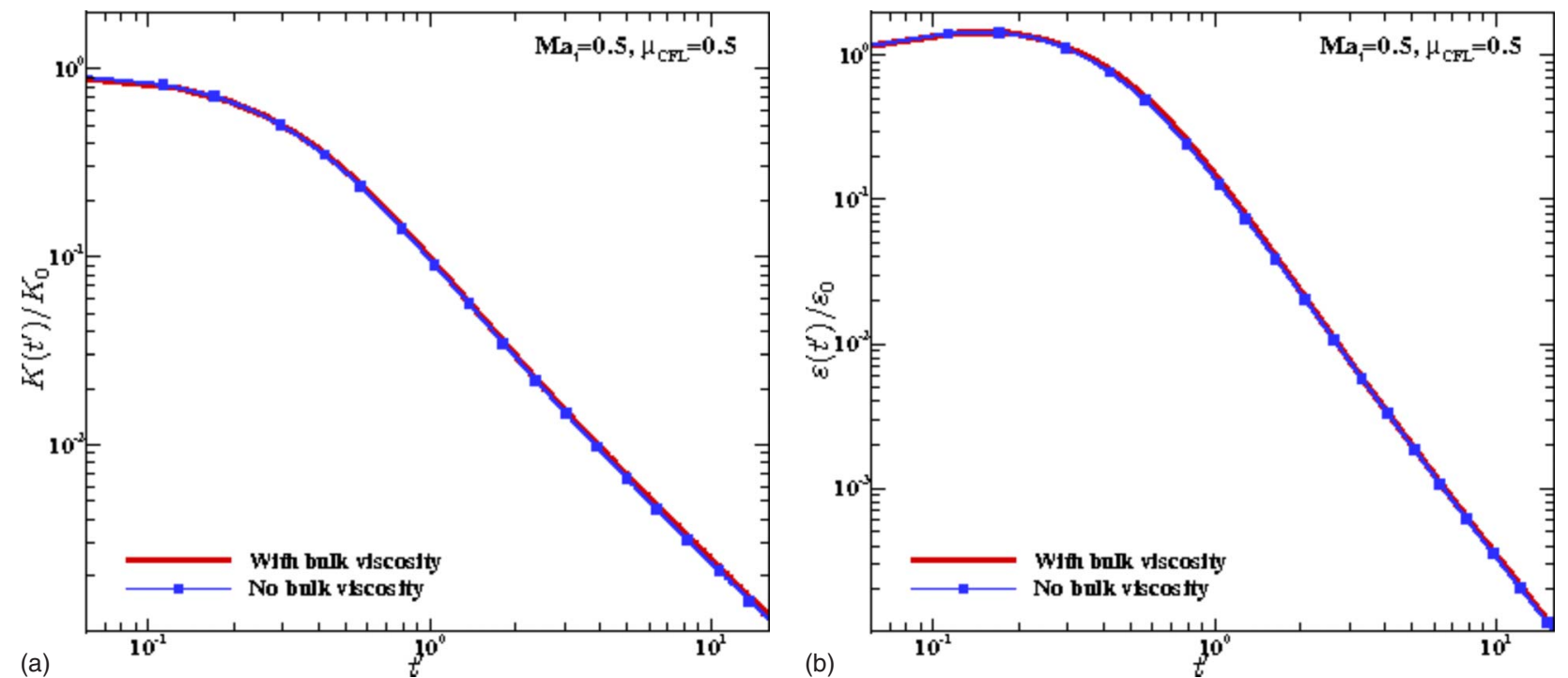

FIG. 25. (Color online) Effect of the bulk viscosity $\eta$ on the kinetic energy $K\left(t^{\prime}\right) / K_{0}$ and the dissipation rate $\varepsilon\left(t^{\prime}\right) / \varepsilon_{0}$. $N^{3}=128^{3}$, $\operatorname{Re}_{\lambda}=72.0, \mathrm{Ma}_{\mathrm{t}}=0.5$, and $\mu_{\mathrm{CFL}}=0.5$.
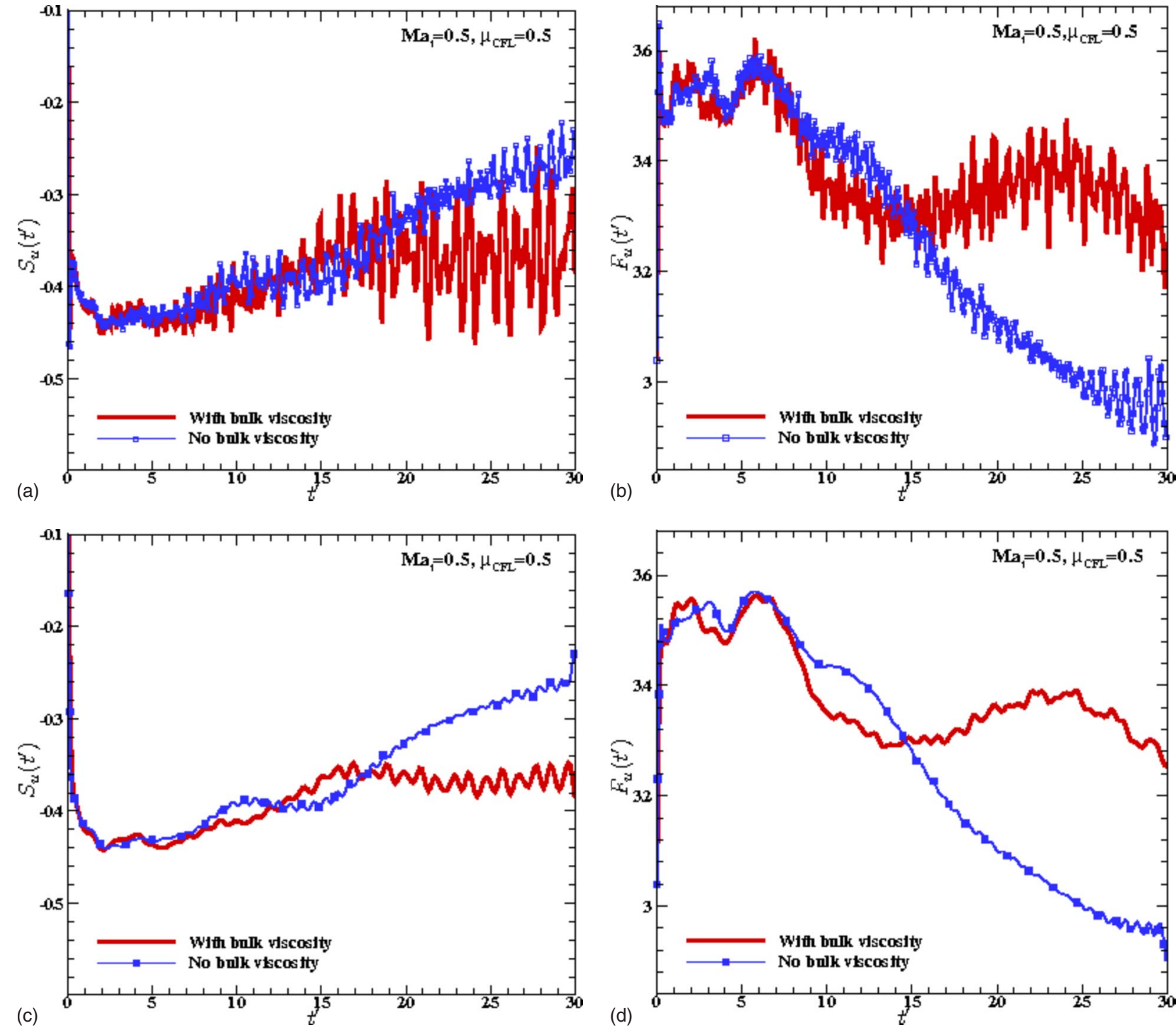

FIG. 26. (Color online) Effect of the bulk viscosity on the skewness $S_{u}(t)$ (left) and the flatness $F_{u}(t)\left(\right.$ right). $N^{3}=128^{3}, \operatorname{Re}_{\lambda}=72.0$, $\mathrm{Ma}_{\mathrm{t}}=0.5$, and $\mu_{\mathrm{CFL}}=0.5$. The bottom figures are the smoothed ones of the top ones. 
macroscopic continuum theory. This feature is particularly relevant to thermochemically nonequilibrium flows for which the Navier-Stokes equations are no longer valid $[39,40,73,74]$. We also note that the full MD-GKS is computationally more intensive for the benefit of better fidelity of flow physics. To fully exploit the advantages and to fully realize the potential of gas-kinetic schemes will remain the subjects of our future research.

\section{ACKNOWLEDGMENTS}

The authors are grateful to an anonymous reviewer whose comments have helped us improve the paper considerably. The authors would like to thank Dr. A. Honein for providing the data of Ref. [13] shown in Fig. 4, and Dr. P. K. Subbareddy and Professor G. V. Candler for providing Ref. [14] to us before its publication. We would also like to acknowledge the funding support from U.S. Department of Defense and Air Force Office of Scientific Research under AFOSR-MURI Project 19 "Hypersonic Transition and Turbulence with Nonequilibrium Thermochemistry" (Dr. J. Schmisseur).
[1] T. Passot and A. Pouquet, J. Fluid Mech. 181, 441 (1987).

[2] S. Kida and S. A. Orszag, J. Sci. Comput. 5, 1 (1990).

[3] S. Kida and S. A. Orszag, J. Sci. Comput. 5, 85 (1990).

[4] S. Lee, S. K. Lele, and P. Moin, Phys. Fluids A 3, 657 (1991).

[5] S. Sarkar, G. Erlebacher, M. Y. Hussaini, and H. O. Kreiss, J. Fluid Mech. 227, 473 (1991).

[6] G. Erlebacher, M. Y. Hussaini, C. G. Speziale, and T. A. Zang, J. Fluid Mech. 238, 155 (1992).

[7] G. A. Blaisdell, N. N. Mansour, and W. C. Reynolds, J. Fluid Mech. 256, 443 (1993).

[8] S. K. Lele, Annu. Rev. Fluid Mech. 26, 211 (1994).

[9] F. A. Jaberi, D. Livescu, and C. K. Madnia, Phys. Fluids 12, 1189 (2000).

[10] R. Samtaney, D. I. Pullin, and B. Kosović, Phys. Fluids 13, 1415 (2001).

[11] H. Foysi, S. Sarkar, and R. Friedrich, J. Fluid Mech. 509, 207 (2004).

[12] D. Livescu and C. K. Madnia, Phys. Fluids 16, 2864 (2004).

[13] A. E. Honein and P. Moin, J. Comput. Phys. 201, 531 (2004).

[14] P. K. Subbareddy and G. V. Candler, J. Comput. Phys. 228, 1347 (2009).

[15] M. Wu and M. P. Martín, AIAA Paper. 2005-4895, 2005.

[16] M. P. Martín, A. J. Smits, M. Wu, and M. Ringuette, AIAA Paper. 2006-0497, 2006.

[17] M. P. Martín, J. Fluid Mech. 570, 347 (2007).

[18] M. Wu and M. P. Martín, AIAA J. 45, 879 (2007).

[19] M. W. Wu and M. P. Martín, J. Fluid Mech. 594, 71 (2008).

[20] J. R. Edwards, Prog. Aerosp. Sci. 44, 447 (2008).

[21] S. K. Godunov, Math. USSR. Sb. 47, 271 (1959).

[22] B. van Leer, J. Comput. Phys. 32, 101 (1979).

[23] D. Drikakis and W. Rider, High-resolution Methods for Incompressible and Low-speed Flows (Springer, Berlin, 2005).

[24] S. K. Lele, J. Comput. Phys. 103, 16 (1992).

[25] M. P. Martín, E. M. Taylor, M. Wu, and V. G. Weirs, J. Comput. Phys. 220, 270 (2006).

[26] E. M. Taylor and M. P. Martín, J. Sci. Comput. 30, 533 (2007).

[27] E. M. Taylor, M. W. Wu, and P. Martín, J. Comput. Phys. 223, 384 (2007).

[28] M. Delanaye and J. A. Essers, AIAA J. 35, 631 (1997).

[29] C. Ollivier-Gooch, A. Nejat, and K. Michalak, AIAA Paper, 2007-4464, 2007.

[30] K. Xu, in The 29th Computational Fluid Dynamics, VKI Lecture Series (The von Karman Institute for Fluid Dynamics, Rhode-St-Genèse, Belgium, 1998), Vol. 1998-03, pp. 1-202.
[31] K. Xu, J. Comput. Phys. 171, 289 (2001).

[32] B. Van Leer, AIAA Paper, 2001-2520, 2001.

[33] K. Xu, J. Comput. Phys. 178, 252 (2002).

[34] T. Ohwada, J. Comput. Phys. 177, 156 (2002).

[35] K. Xu, M. L. Mao, and L. Tang, J. Comput. Phys. 203, 405 (2005).

[36] T. Ohwada and S. Fukata, J. Comput. Phys. 211, 424 (2006).

[37] G. May, B. Srinivasan, and A. Jameson, J. Comput. Phys. 220 , 856 (2007).

[38] Q. B. Li and S. Fu, J. Comput. Phys. 220, 532 (2006).

[39] W. Liao, L.-S. Luo, and K. Xu, J. Rocket and Spacecraft 44, 1232 (2007).

[40] W. Liao, Y. Peng, L.-S. Luo, and K. Xu, Prog. Comput. Fluid Dym. 8, 97 (2008).

[41] K. Xu, X. He, and C. P. Cai, J. Comput. Phys. 227, 6779 (2008).

[42] K. Xu and L. Tang, Phys. Fluids 16, 3824 (2004).

[43] K. Xu and E. Josyula, Phys. Rev. E 71, 056308 (2005).

[44] K. Xu and E. Josyula, Comm. Comp. Phys. 1, 425 (2006).

[45] C. P. Cai, D. D. Liu, and K. Xu, AIAA J. 46, 1054 (2008).

[46] Q. B. Li, H. X. Chen, and S. Fu, Phys. Fluids 15, 3240 (2003).

[47] Q. B. Li and S. Fu, Comput. Fluids 32, 1357 (2003).

[48] Q. B. Li, S. Fu, and K. Xu, J. Comput. Phys. 204, 692 (2005).

[49] S. Fu and Q. B. Li, Int. J. Heat Fluid Flow 27, 895 (2006).

[50] K. Xu, J. Comput. Phys. 134, 122 (1997).

[51] Y. S. Lian and K. Xu, J. Comput. Phys. 163, 349 (2000).

[52] Y. S. Lian and K. Xu, Comput. Fluids 29, 725 (2000).

[53] T. Ohwada and K. Xu, J. Comput. Phys. 201, 315 (2004).

[54] K. Xu and Z. H. Li, J. Fluid Mech. 513, 87 (2004).

[55] K. Xu, J. Comput. Phys. 153, 334 (1999).

[56] H. Z. Tang and K. Xu, J. Comput. Phys. 165, 69 (2000).

[57] K. Xu, J. Comput. Phys. 178, 533 (2002).

[58] H. Z. Tang, T. Tang, and K. Xu, Z. Angew. Math. Phys. 55, 365 (2004).

[59] M. S. Ghidaoui, A. A. Kolyshkin, J. H. Liang, F. C. Chan, Q. Li, and K. Xu, J. Fluid Mech. 548, 309 (2006).

[60] Y. T. Que and K. Xu, Int. J. Numer. Methods Fluids 50, 1003 (2006).

[61] W. Liao, Y. Peng, and L.-S. Luo, AIAA Paper, 2008-0548, 2008.

[62] S. Harris, An Introduction to the Theory of Boltzmann Equation (Holt, Rinehart and Winston, New York, 1971; reprinted by Dover, New York, 2004).

[63] P. Bhatnagar, E. Gross, and M. Krook, Phys. Rev. 94, 511 
(1954).

[64] M. N. Kogan, Rarefield Gas Dynamics (Plenum Press, New York, 1969).

[65] Z. L. Guo, H. W. Liu, L.-S. Luo, and K. Xu, J. Comput. Phys. 227, 4955 (2008).

[66] B. van Leer, J. Comput. Phys. 14, 361 (1974).

[67] Q. B. Li, S. Fu, and K. Xu, AIAA J. 43, 2170 (2005).

[68] K. Xu and X. Y. He, J. Comput. Phys. 190, 100 (2003).

[69] R. S. Rogallo, NASA, Technical Report No. TM-8135, 1981 (unpublished).
[70] S. B. Pope, Turbulent Flows (Cambridge University Press, Cambridge, England, 2000).

[71] T. T. Clark and C. Zemarch, Phys. Fluids 10, 2846 (1998).

[72] Y. Peng, W. Liao, L.-S. Luo, and L.-P. Wang, Comput. Fluids (unpublished).

[73] J. D. Anderson, Hypersonic and High Temperature Gas Dynamics (McGraw-Hill, New York, 1989; reprinted by AIAA, Reston, VA, 2000).

[74] W. Vincenti and C. Krueger, Introduction to Physical Gas Dynamics (Krieger Wiley, New York, 1965). 